\title{
EVOLUTIONARY DYNAMICS IN CHANGING ENVIRONMENTS
}

\section{Dissertation}

to acquire the doctoral degree in mathematics and natural science 'Doctor rerum naturalium' at the Georg-August-Universität Göttingen in the doctoral degree programme 'Physics of Biological and Complex Systems' at the Georg-August University School of Science (GAUSS)

\author{
Submitted by \\ Frank Stollmeier \\ from Lippstadt, Germany \\ Göttingen, 2018
}




\section{Thesis Committee:}

- Prof. Dr. Marc Timme (First Referee)

Institute for Theoretical Physics, Technical University of Dresden

- Prof. Dr. Theo Geisel (Second Referee)

Max Planck Institute for Dynamics and Self-Organization

- Dr. Jan Nagler (Supervisor)

Computational Physics for Engineering Materials, IfB, ETH Zurich

- Prof. Dr. Ulrich Parlitz

Max Planck Institute for Dynamics and Self-Organization

\section{Further members of the examination board:}

- Prof. Dr. Stefan Klumpp

Institute for Nonlinear Dynamics, University of Göttingen

- Prof. Dr. Reiner Kree

Institute for Theoretical Physics, University of Göttingen

Date of the oral examination: April 19, 2018 
RELATED PUBLICATIONS

A F. Stollmeier and J. Nagler. Unfair and Anomalous Evolutionary Dynamics from Fluctuating Payoffs. In: Physical Review Letters 120.5 (2018), p. 058101.

B F. Stollmeier. [Re] A simple rule for the evolution of cooperation on graphs and social networks. In: ReScience 3.1 (2017).

C F. Stollmeier and J. Nagler. Phenospace and mutational stability of shared antibiotic resistance. Submitted to Physical Review X.

Chapter 3 is based on publication A, chapter 4.3 is based on publication B, and chapter 5 is based on submitted paper $\mathbf{C}$. Each chapter also includes some additional content and the texts, the equations and the figures in these chapters are modified and partly rearranged compared to the publications. 



\section{CONTENTS}

I INTRODUCTION I

1.1 Motivation ............... I

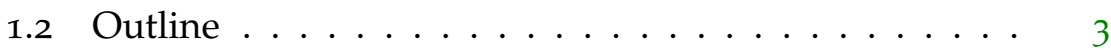

2 FUNDAMENTALS OF EVOLUTIONARY GAME THEORY 5

2.1 Unexpected results of Darwinian evolution ...... 5

2.2 Evolutionarily stable state ............ 6

2.3 Replicator equation ............. 8

2.4 Frequency-dependent Moran process . . . . . . . . 9

2.5 Evolutionary games ............. 10

3 EVOLUTIONARY GAME THEORY WITH PAYOFF FLUCTUA$\begin{array}{ll}\text { TIONS } & 15\end{array}$

3.1 Introduction ................ 15

3.2 Anomalous evolutionarily stable states ....... 16

3.3 Deterministic payoff fluctuations ......... 20

3.4 Classification of games with payoff fluctuations . . . . 22

3.5 Discussion ................ 27

3.6 Proofs and methods ............. 28

3.6.1 Approximation of the geometric mean . . . 28

3.6.2 Stochastic payoff fluctuations . . . . . . . 28

3.6.3 Correspondence of payoff rank criteria and generalized criteria with constant payoffs . . . . 33

4 EVOLUTIONARY GAMES ON NETWORKS 37

4.1 Introduction .............. 37

4.2 Existing results on structured populations indicate effects of inherent noise . . . . . . . . . . 37

4.3 Implementation of evolutionary games on networks . . 38

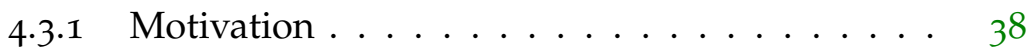

4.3.2 Short review of Ohtsuki et al.: A simple rule for the evolution of cooperation on graphs and social networks (Nature, 2006) . . . . . . . . . 39

4.3.3 Description of algorithms ........ 40

4.3.4 Comparison of own implementation with results in the paper by Ohtsuki et al. . . . . . . . $4^{1}$

4.4 The effect of inherent payoff noise on evolutionary dy-

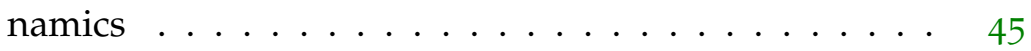

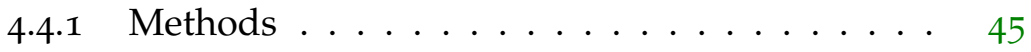

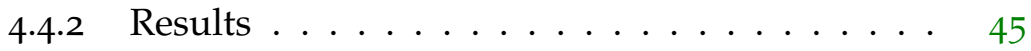

4.5 Discussion ................. 47

5 SHARED ANTIBIOTIC RESISTANCE 49

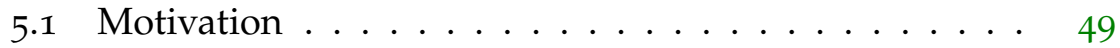

5.2 Introduction .................. 5 
5.3 The phenospace of antibiotic resistance . . . . . 51

5.4 Cell model . . . . . . . . . . . . . . . . . 52

5.5 Colony model . . . . . . . . . . . . . . 53

5.6 Fitness landscape . . . . . . . . . . . . . . 55

5.7 Evolutionary dynamics . . . . . . . . . . . . 55

$5 \cdot 7.1$ Strain A alone . . . . . . . . . . . . . . . 55

5.7 .2 Strain A invaded by B . . . . . . . . . . 55

$5 \cdot 7 \cdot 3$ Strain A and B, invaded by C . . . . . . . 57

5.8 Mutational stability and the direction of evolution . . $\quad 58$

5.9 Conclusion . . . . . . . . . . . . . 60

5.10 Outlook.................... 61

6 AdAPtation to Fluctuating temperatures 63

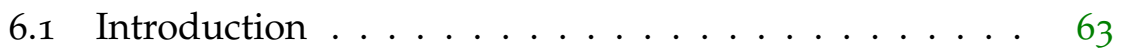

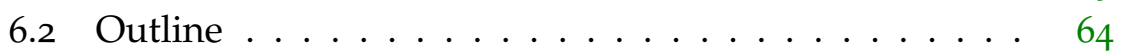

6.3 Theoretical examples . . . . . . . . . . . . . 64

6.3.1 Periodically changing temperature ....... 64

6.3.2 Randomly fluctuating temperature . . . . . . 67

6.4 Modeling the growth of nematodes $\ldots \ldots \ldots 70$

6.4.1 Development .............. 70

6.4 .2 Reproduction . . . . . . . . . . . 72

6.4 .3 Population growth ............. 75

6.4.4 Growth rates at constant temperatures . . . . 76

6.4.5 Growth rates with switching temperatures . . . 77

6.5 Weather data . . . . . . . . . . . . . . 79

6.6 Estimation of soil temperatures . . . . . . . . 80

6.7 Results . . . . . . . . . . . . . 82

6.8 Discussion ........................ 89

7 CONCLUSION 91

7.1 Summary and Discussion ................. 91

7.1.1 Overview ............... 91

7.1.2 Chapter 3: Evolutionary game theory with payoff fluctuations . . . . . . . . . . . 92

7.1.3 Chapter 4: Evolutionary games on networks . . 93

7.1.4 Chapter 5: Shared antibiotic resistance ..... 93

7.1.5 Chapter 6: Adaptation to fluctuating temperatures 94

7.2 Outlook: Towards applied evolutionary dynamics . . . 95

$\begin{array}{lll}\text { A APPENDIX } & 97\end{array}$

A.1 Parameters for figure $5.4 \ldots \ldots \ldots \ldots 7$

A.2 Metadata and results of weather data analysis . . . 99

$\begin{array}{ll}\text { BIBLIOGRAPHY } & 103\end{array}$

$\begin{array}{ll}\text { ACKNOWLEDGMENTS } & 113\end{array}$ 


\section{INTRODUCTION}

\subsection{MOTIVATION}

The aim of evolutionary dynamics is to describe how species change. It emerged after two fundamental insights. First, that species change at all, and second, that this happens due to variation, heredity and natural selection. Both have been controversially debated, but since Charles Darwin presented a line of arguments with a large number of supporting observations these insights became the established theory of evolution. Which open questions remain after these underlying mechanisms of evolution are discovered? Can we calculate the course of evolution using a set of basic laws for variation, heredity and natural selection?

The starting point of such calculations is the fitness function, which is defined as the reproductive success of an organism. The fitness depends first of all on the phenotype of the organism. This part of the fitness function, often metaphorically referred to as the "fitness landscape", can be considered as the potential of evolutionary dynamics and we can obtain the direction of evolution from the gradient of the fitness with respect to the phenotype. While the species evolves, the fitness landscape changes dynamically, because the fitness also depends on the environmental conditions, on the interactions with other individuals of the same species and on the interactions with other individuals of other evolving species. At the same time, the individuals of the species also have an impact on the environment and on the fitness of individuals of other species.

Taken together, these coupled fitness functions constitute a complex, nonlinear, high dimensional, heterogeneous, stochastic system. Even if we knew all relevant biological mechanisms, seemingly simple questions remain challenging problems. For example, which conditions lead to extinction or speciation? Or how many species can coexist and how stable is such a system of coexisting species? The literature contains already a great number of answers, but each relies on a very specific set of assumptions.

For example, let us assume a homogeneous habitat with species whose fitness depends only on their own phenotype and on the environmental conditions. We further assume that the environmental conditions 
do not change and that the populations are infinitely large. Since the species with the highest fitness outcompetes all species with a lower fitness regardless how small the advantage is, only one species can survive under these assumptions. This reasoning is known as the competitive exclusion principle. However, the relevance for natural systems is very limited, because the assumptions are almost never satisfied. A famous case is the high diversity of phytoplankton species in ocean water. Due to the supposed homogeneity of ocean water and the limited number of resources, the surprisingly high diversity of phytoplankton species in ocean water is called the "paradox of the plankton". Today, it is known that none of the aforementioned assumptions is satisfied for phytoplankton in ocean water, so the competitive exclusion principle does not apply to this case [1].

We can extend the example by assuming that the fitness of a species also depends on the phenotype of other species. To keep it simple, we further assume that the individuals interact with equal probability with every other individual, and that no new mutations occur except for those that are already present at the beginning. The resulting coupled evolutionary dynamics is the subject of evolutionary game theory. Imagine two species which each have a high fitness if their population is small and a low fitness if their population is large compared to the population of the other species. These two species can coexist, because natural selection always favors the species that is rare compared to the other species, which leads to an evolutionarily stable state at the ratio of populations for which both species have an equal fitness.

These typical assumptions for evolutionary game theory are still far from being realistic. Changing only one of them can lead to completely different results, but can also make it difficult or even impossible to find exact solutions. Hence, almost any attempt to relax one assumption has resulted in a new direction of research, e. g. evolutionary game theory with finite instead of infinite populations [2-11], with populations on networks or spatially distributed populations instead of well-mixed populations [12-19] or with mutations occurring during the process $[7,20,21]$. The attempts to replace the assumption of a constant environment with variable environments headed in different directions because the environment can affect several parameters in the system. For example, the environment may affect the selection strength [8], the reproduction rate [22-25], or the population size [10, 11, 26-28] for each species independently. Typical properties of interest in these studies are the probability of extinction or fixation of a mutation and the mean time to extinction or fixation of a mutation $[3,5,8,9,27,29-43]$.

In this thesis, we aim to study the effects of changing environments on evolutionary dynamics in a different way. We assume that the fitness of species changes depending on the environmental fluctua- 
tions and ask how this affects the stationary states of evolutionary processes. In general, it is known that noise in a dynamical system can change or create stationary states [44-48], but the implications of these effects in evolutionary dynamics are largely unknown.

\subsection{OUTLINE}

In the first half of this thesis we study the effects caused by environmental fluctuations in evolutionary game theory.

In chapter 2 we review some fundamental concepts of evolutionary game theory that we use in the following chapter.

In chapter 3, we analyze the stationary states in evolutionary game theory if the values in the payoff matrix, which describes how the species interact with and depend on each other, change continuously. We find that the positions, the number and the stability of the stationary states change depending on the intensity and the kind of fluctuations. We show that these changes can be interpreted as transitions between evolutionary games caused by payoff fluctuations.

In chapter 4 , we show that evolutionary games on networks and in spatially distributed populations without payoff fluctuations show similar effects as those that we discovered in chapter 3 . We show that this allows a new perspective on key results of evolutionary games in structured populations, in particular how the network type, the degree distribution and migration affect the evolution of cooperation.

The classical models of evolutionary game theory that we study in the first half can not be tested experimentally. These models were developed to demonstrate qualitative differences, but not to make predictions for any real biological system. In the second half of this thesis we develop two different models for evolutionary processes of microbiological species that can be tested experimentally.

In chapter 5, we develop a model for the evolution of shared antibiotic resistance in bacteria. This type of resistance has been observed in experiments and suggested as a real-world example of evolutionary game theory. Our model successfully reproduces a number of observations from experiments on the evolution of antibiotic resistance and allows new insights in the evolutionary dynamics and the phenospace of antibiotic resistance. However, for understanding the impact of changing environments, e.g. a fluctuating antibiotic concentration, it remains a first step because predictions for changing environmental conditions require to know the absolute fitness functions which have to be measured experimentally.

In chapter 6, we develop a model for the adaptation of nematodes to temperature fluctuations. Here, the absolute fitness functions are already partly known from experiments. This allows us to make predictions that can be compared to measurements with nematode strains from natural habitats of different local climates. 



\section{2}

FUNDAMENTALS OF EVOLUTIONARY GAME THEORY

\subsection{UNEXPECTED RESULTS OF DARWINIAN EVOLUTION}

Many animals in the wild have to fight with other individuals of the same species for food, territory, social status or mates. If we assume that individuals which are successful in these contests have a higher chance to reproduce, natural selection should favor traits that give an advantage in such contests, leading to

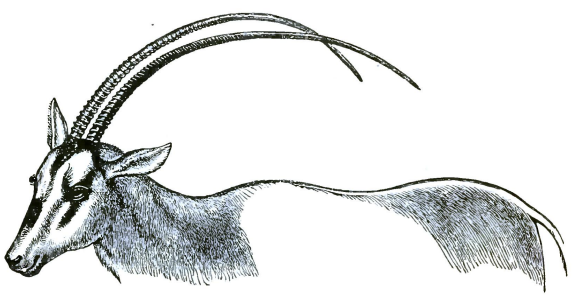

Figure 2.1: Oryx leucoryx (male). Image: Charles Darwin, 1871 (public domain) [49]. an evolutionary arms race for strength, effective weapons and aggressive behavior. However, such intraspecific conflicts are often surprisingly harmless. For example, male deers fight with each other by crashing and pushing their antlers, but usually refrain from attacking their opponent from an unprotected side. Also males of many species among the antelopes and goats attack each other with their horns, but the shape of their horns does not seem to be optimized to harm others (see figure 2.1). How can such ritualized tactics and inefficient weapons be the result of Darwinian evolution?

Evidently, a species with less intraspecific aggression has an advantage over a species with more intraspecific aggression. This argument could explain limited intraspecific aggression if we assume that natural selection acts as "group selection". However, group selection has been debated controversially, because natural selection primarily acts on the individual level.

But if we assume that aggressive individuals have an advantage over non-aggressive individuals and that natural selection acts on the individual level, why do intraspecific conflicts not necessarily result in an evolutionary arms race? 


\subsection{EVOLUTIONARILY STABLE STATE}

In 1973, John Maynard Smith and Georg Price published a paper on "The Logic of Animal Conflict", where they combined Darwinian evolution with game theory [50]. They assumed that a population consists of individuals that use inherited strategies and that the success of these individuals depends not only on their own strategy but also on which strategies the other individuals use. For the sake of simplicity, we can consider a population in which only two strategies are present, one aggressive strategy and one non-aggressive strategy. We call individuals "hawks" if they use the aggressive strategy and "doves" if they use the non-aggressive strategy. These are symbolic names for the strategies, the individuals could still be deers, antelopes or any other species. If two hawks get into a conflict, they fight with each other, hence they pay a cost $C$ in form of energy and risk of injuries. Each of them has a $50 \%$ chance to win the disputed resource of value $V$, where we assume that $C>V>0$. If two doves meet at the resource, they do not fight with each other but just share the resource. If a hawk meets with a dove, the dove retires and leaves the resource to the hawk. The payoff received by the individuals depending on the combination of strategies can be written as a payoff matrix,

$$
\begin{aligned}
& \text { Hawk Dove }
\end{aligned}
$$

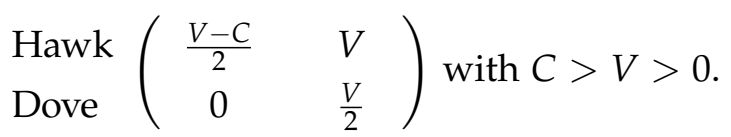

Using classical game theory we could analyze which strategy a rational player should choose to maximize the payoff under the assumption that the opponent tries to maximize its payoff as well. In contrast, in evolutionary game theory there is no rational choice about the decision, because the strategies are inheritable phenotypes. The strategies in the population change only due to natural selection.

Let us assume that the population is infinitely large and describe the state of the population as $\mathbf{x}=\left(x_{H}, x_{D}\right)$, where $x_{H}$ is the share of hawks and $x_{D}=1-x_{H}$ is the share of doves in the population. If the individuals get into conflicts with random other individuals, the average payoff of the hawks is $P_{H}=\frac{V-C}{2} x_{H}+V x_{D}$ and the average payoff of the doves is $P_{D}=0 x_{H}+\frac{V}{2} x_{D}$. In short, the average received payoff is $\left(P_{H}, P_{D}\right)=M \mathbf{x}$, where $M$ is the payoff matrix 2.1.

Consider a population of doves in which suddenly one hawk appears due to a mutation. This hawk receives a higher average payoff than the doves, because it wins every conflict without having to pay the costs for fighting. Hence, natural selection favors the hawk strategy and the population share $x_{H}$ increases. At the same time the increase of the population share of hawks reduces the advantage of hawks, until the population approaches a state in which hawks and doves 


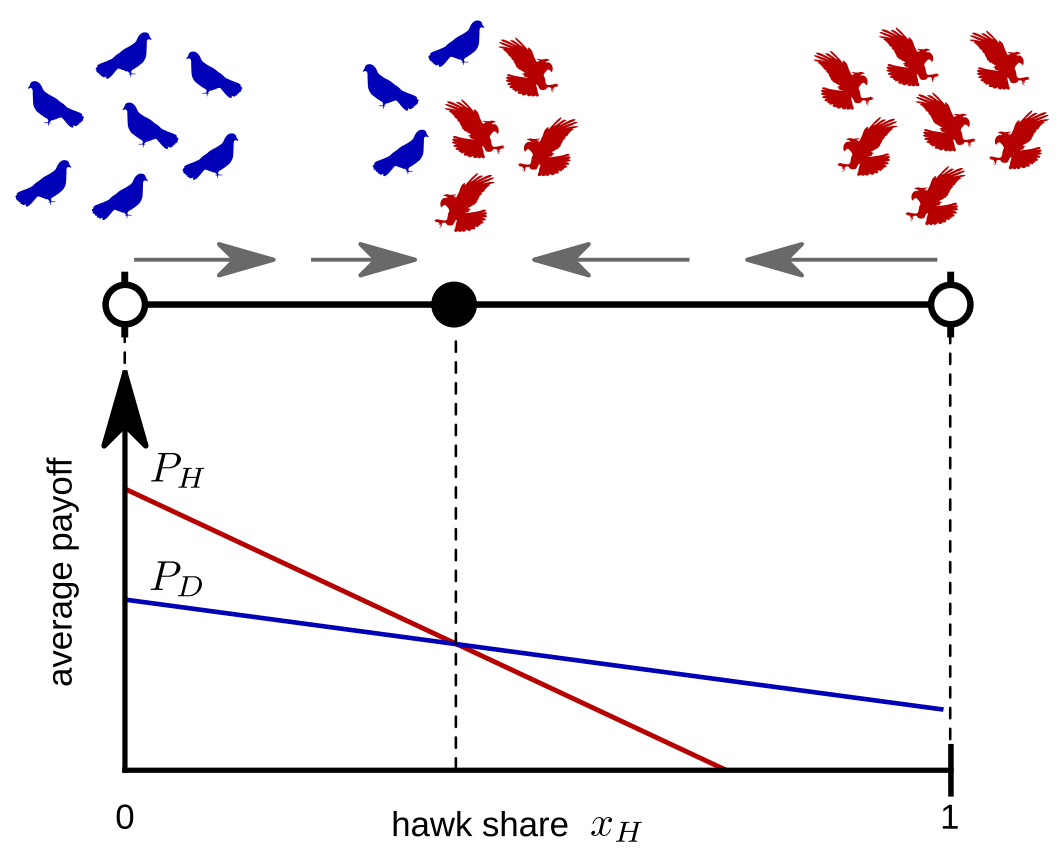

Figure 2.2: Illustration of the evolutionarily stable state. In a Hawk-Dove game, the strategy which is rare compared to the other strategy has a higher payoff. The rare strategy is favored by natural selection until the population approaches the evolutionarily stable state, where both strategies receive the same payoff.

receive equal average payoffs. The situation is reversed in a population of hawks in which a mutation causes one individual to be a dove. The payoff of the dove is zero, but the payoff of the hawks is negative because the costs $C$ for fighting are larger than the value $V$ of the resources. Hence, natural selection favors the doves until the population approaches the state in which both receive the same average payoff.

Consequently, for all initial states $0<x_{H}<1$ the evolutionary process approaches the state where $P_{H}=P_{D}$, which is called the evolutionarily stable state (see figure 2.2).

The Hawk-Dove game shows that natural selection does not always favor the individuals with the more aggressive strategies. Aggressive and non-aggressive individuals can coexist in an evolutionarily stable state. However, in the introductory examples of intraspecific conflicts between male deers or between male antelopes there are no two types of deers with different tactics or two types of antelopes with different horn shapes. Does the idea of hawks and doves which coexist in an evolutionarily stable state apply to these examples?

The strategies can be interpreted in two different ways. If an individual is either hawk or dove, they are called "pure strategies". If both strategies are present in every single individual and strategy $i$ is applied to a degree or with the probability $x_{i}$, they are called "mixed strategies". The male deers and antelopes are examples of 
A

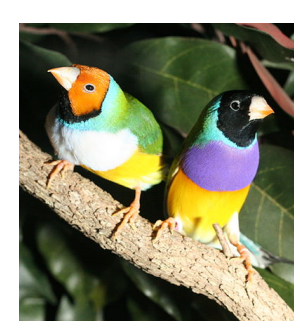

B

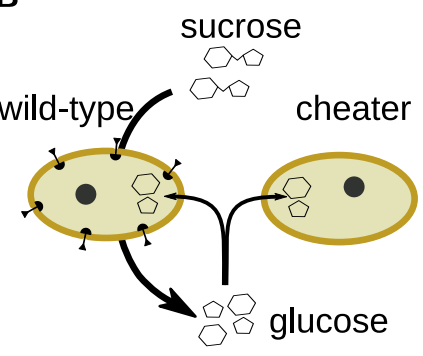

C

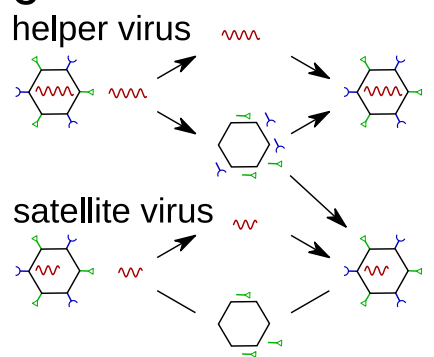

Figure 2.3: Examples of Hawk-Dove games in nature.

(A) The head-color polymorphism of Gouldian Finches correlates with their hormone profile. In competitions, e. g. for the best nest locations, the individuals with red heads behave more aggressively than the individuals with black heads [51]. Image: Nigel Jacques, "GouldianFinches", CC-BY-SA 2.5.

(B) Yeast cells preferably consume glucose, but if only sucrose is available, the wild-type can hydrolyze sucrose to glucose. Cheater cells do not invest energy in producing glucose, but consume the glucose that the wild-type loses due to diffusion [52].

(C) A satellite virus can not produce all necessary components to reproduce itself. But if the host cell is also infected by the corresponding helper virus, the satellite virus can use components produced by the helper virus to reproduce [53].

mixed strategies. Here, the evolutionarily stable state is not a coexistence of two types of individuals but rather a compromise between being to some degree aggressive and to some degree harmless. For biological examples of Hawk-Dove games with pure strategies see figure 2.3.

\subsection{REPLICATOR EQUATION}

In 1978, Peter Taylor and Leo Jonker formalized the idea of evolutionary game theory [54]. They derived a coupled differential equation, called "replicator equation", which describes how the populations of strategies change depending on the fitness differences between strategies. In general, the replicator equation can be written as

$$
\dot{x}_{i}=x_{i}\left(f_{i}(\mathbf{x})-\langle f\rangle\right),
$$

where $x_{i}$ is the population share of strategy $i, f_{i}$ is the average fitness of individuals with strategy $i$, and $\langle f\rangle$ is the average fitness of all individuals in the population. For games that are described by a payoff matrix $M$ the replicator equation simplifies to

$$
\dot{x}_{i}=x_{i}\left((M \mathbf{x})_{i}-\mathbf{x}^{\mathrm{T}} M \mathbf{x}\right)
$$


where the vector $\mathbf{x}=\left\{x_{i}\right\}$ describes the population shares.

Setting the right hand side zero yields the fixed points of the evolutionary game. For example, using the payoff matrix 2.1 for the HawkDove game, the replicator equation has three fixed points. The two fixed points at $x_{H}=0$ (no hawks) and $x_{H}=1$ (no doves) are unstable and the fixed point at $x_{H}=\frac{V}{C}$ (coexistence of hawks and doves) is the evolutionarily stable state.

\subsection{FREQUENCY-DEPENDENT MORAN PROCESS}

The replicator equation has the limitation that it describes a deterministic process, because the populations are assumed to be infinite. Due to the finite size of real populations, it is more realistic to describe evolutionary games as stochastic processes. A formalization of the idea of evolutionary game theory as a stochastic process is the frequency-dependent Moran process [3, 55]. Consider a population of finite size $N$, which consists of $N_{1}$ individuals with strategy 1 and $N_{2}=N-N_{1}$ individuals of strategy 2. If the payoff matrix of the game is given by $M=[a, b, c, d]$ and each individual plays one game against every other individual, they receive the average payoff

$$
\begin{aligned}
& P_{1}\left(N_{1}\right)=\frac{a\left(N_{1}-1\right)+b\left(N-N_{1}\right)}{N-1} \\
& P_{2}\left(N_{1}\right)=\frac{c N_{1}+d\left(N-N_{1}-1\right)}{N-1} .
\end{aligned}
$$

Since the considered game may be just one among many contributions to the total fitness of the individual, the fitness of strategy $i$ is defined as

$$
f_{i}=1-w+w P_{i}
$$

where $w$ is called the selection strength. The selection strength is often assumed to be small $w \ll 1$, which is called "weak selection".

At every time step, one of the individuals is randomly chosen to reproduce, where the probability is proportional to the fitness of the individuals. Next, one individual is randomly chosen to die, where the probabilities are the same for all individuals. The result is a birthdeath process in which the size of the population remains $N$, but at every time step the number of individuals with strategy 1 or 2 may increase by one, decrease by one, or remain constant. The transition probability that $N_{1}$ increases (decreases) by one is the product of the probability that an individual of strategy 1 (2) is chosen to reproduce 
and the probability that an individual of strategy 2 (1) is chosen to die,

$$
\begin{aligned}
T_{N_{1}, N_{1}-1} & =\frac{f_{2}\left(N_{1}\right)\left(N-N_{1}\right)}{f_{2}\left(N_{1}\right) \cdot N_{1}+f_{2}\left(N_{1}\right)\left(N-N_{1}\right)} \cdot \frac{N_{1}}{N} \\
T_{N_{1}, N_{1}} & =1-T_{N_{1}, N_{1}-1}-T_{N_{1}, N_{1}+1} \\
T_{N_{1}, N_{1}+1} & =\frac{f_{2}\left(N_{1}\right) \cdot N_{1}}{f_{2}\left(N_{1}\right) \cdot N_{1}+f_{2}\left(N_{1}\right)\left(N-N_{1}\right)} \cdot \frac{N-N_{1}}{N} .
\end{aligned}
$$

With the Moran process we can for example analyze how likely it is that a mutation that occurred in only one individual spreads to the whole population, in other words, that the species evolved by one mutation. The event that the process ends at $N_{1}=N$ if it started at $N_{1}=1$ is called the fixation of strategy 1 . From the transition probabilities it is possible to calculate the fixation probability and the expected time to fixation $[3,6]$.

In the limit of infinite populations $N \rightarrow \infty$, the frequency-dependent Moran process converges to the replicator equation [4].

\subsection{EVOLUTIONARY GAMES}

The Hawk-Dove game that we discussed in section 2.2 belongs to the class of $2 \times 2$-matrix games, which are the games that consist of two opponents with two strategies. In general, matrix games can also consist of more than two opponents and more than two strategies. Beyond matrix games, a game can also be defined by any payoff function $P(s, \mathbf{x})$ that maps a strategy $s$ of an individual and a state $\mathbf{x}$ of the population to a payoff value for the individual. For example, in chapter 5 we develop an evolutionary game based on a payoff function $P(s, \mathbf{x})$ to model the interaction between antibiotic resistant and antibiotic sensitive bacteria cells.

The number of games is infinte and theoretically every interaction between individuals can be described as a matrix game or as a payoff function game. Only for the class of $2 \times 2$-matrix games we can get an overview of the possible games. The $2 \times 2$-matrix games are traditionally distinguished based on the rank of the four values in their payoff matrix [56-58]. For simplicity, we use the four values 1, 2, 3, and 4 as payoff values and to define the ranks of the games. For instance, if we say that a game has the payoff matrix $M=[2,3,1,4]$, we mean that this payoff matrix is an example of this game and that this game is defined by all payoff matrices with the same ranks, in this case all matrices $M=[a, b, c, d]$ where $c<a<b<d$.

If we ignore "tie games", in which two payoff values are equal, 24 matrices with different ranks of values can be created by swapping pairs of values in the payoff matrix. Due to the symmetry, that swapping the two rows and the two columns of the matrix leads to the same 
game just with a different order of the strategies, there are 12 distinct $2 \times 2$-matrix games.

Here, we introduce six of these twelve games, because these six games appear in chapter 3 and chapter 4 . For illustrations of the dynamics and the received payoffs in these games see figure 2.4.

Prisoner's Dilemma: The first and certainly most famous game is the Prisoner's Dilemma, which is used as a prototype model for the evolution of cooperation. The payoff matrix can be written as

Cooperate
Defect $\left(\begin{array}{cc}\text { Cooperate } & \text { Defect } \\ 3 & 1 \\ 4 & 2\end{array}\right)$.

If all individuals cooperate they receive a relatively high payoff. The dilemma arises from the fact that an individual that defects always receives a higher payoff than an individual that cooperates. Hence, natural selection always favors the defectors, which leads to the extinction of the cooperators and a relatively low payoff for the defectors.

Typical situations of Prisoner's Dilemmas consist of an individual which can invest time or energy or take a risk to do something that benefits another individual, for example providing the other individual with food or protection.

The game is similar to the Hawk-Dove game as introduced in section 2.2, payoff matrix 2.1, but with $V>C$ instead of $C>V$. Therefore, some biological examples, can be either a Hawk-Dove game or a Prisoner's Dilemma, depending on the costs and the values, such as the interaction between a helper virus and a satellite virus shown in figure $2.3 \mathrm{C}[53]$.

Harmony: The Harmony game is a model for agreement without a conflict. The payoff matrix can be written as

Cooperate
Defect $\left(\begin{array}{cc}\text { Cooperate } & \text { Defect } \\ 4 & 3 \\ 2 & 1\end{array}\right)$.

The Harmony game can be considered the opposite of the Prisoner's Dilemma, because in this game the cooperators always receive a higher payoff than the defectors. This leads to one evolutionarily stable state in which all individuals cooperate.

An example could be individuals which form a crowd to protect against predators or to shelter from the cold, because both sides benefit from joining the crowd. 
Hawk-Dove game: We introduced the Hawk-Dove game in section 2.2 using a parametrization of the payoff matrix with the two variables $V$ and $C$. In general, all Hawk-Dove games have a payoff matrix with the same ranks as the matrix

$$
\begin{aligned}
& \text { Hawk } \\
& \text { Dove }
\end{aligned}\left(\begin{array}{cc}
1 & 4 \\
2 & 3
\end{array}\right) \text {. }
$$

For biological examples see section 2.2 and figure 2.3.

Stag Hunt: The Stag Hunt game is a model for coordination and trust. The payoff matrix of a Stag Hunt game is

$$
\begin{aligned}
& \text { Stag Hare } \\
& \text { Hare }\left(\begin{array}{ll}
4 & 1 \\
3 & 2
\end{array}\right) \text {. }
\end{aligned}
$$

If two individuals coordinate their efforts, they are able to hunt a stag and receive a high payoff. If the two individuals work alone, they are able to hunt a hare and receive a lower payoff. If one individual aims for the stag and the other individual for the hare, only the second individual is successful. This game has two evolutionarily stable states, one state where all individuals coordinate their efforts to hunt for the stag and one where all individuals work alone to hunt for the hare. A third stationary state, in which both strategies coexist, is unstable, because with one additional individual hunting for stags the strategy of hunting for stags becomes more successful and vice versa.

As the name of the game suggests, typical situations of this game are predators hunting for prey.

Battle: The Battle game is a model for anti-coordination with a conflict of interests. The payoff matrix can be written as

$$
\begin{aligned}
& \text { Leader } \\
& \text { Supporter }
\end{aligned}\left(\begin{array}{cc}
1 & \text { Supporter } \\
3 & 2
\end{array}\right) \text {. }
$$

If two individuals of the same strategy meet, they receive a relatively low payoff. If two individuals of different strategies meet, they receive a relatively high payoff. The strategy that is the minority has an advantage over the other strategy because it has the higher chance to meet a partner with the opposite strategy. The leads to an evolutionarily stable state where both strategies coexist. The evolutionary dynamics of the Hawk-Dove game and the Battle game is qualitatively the same, both have two unstable fixed points where only one 
strategy exists and an evolutionarily stable state where both strategies coexist. The difference between these two games is that in the Hawk-Dove game both strategies benefit from an increased number of doves, but in the Battle game only leaders benefit from an increased number of supporters and only supporters benefit from an increased number of leaders.

Typical situations of Battle games are cases where division of labor is an advantage for both partners, but the tasks require different effort, such like different roles of males and females in reproduction or parental care.

Hero: The Hero game another model for anti-coordination with a conflict of interests. The payoff matrix can be written as

$$
\begin{aligned}
& \text { Leader } \\
& \text { Supporter }
\end{aligned}\left(\begin{array}{cc}
2 & 4 \\
3 & 1
\end{array}\right) \text {. }
$$

This game is very similar to the Battle game. The only difference is that here two leaders receive a larger payoff than two supporters, while in the Battle game two supporters receive a larger payoff than two leaders. In classical game theory, this changes the incentives to switch the own strategy if the opponent maintains its strategy. But in an evolutionary game with two strategies, the share of one strategy can not change independently of the share of the other strategy. The evolutionary dynamics of the two games has the same structure and the received payoff of the two strategies in both games change in the same directions depending on the population shares (compare figure ${ }_{2.4} \mathrm{E}$ and $2.4 \mathrm{~F}$ ), hence we call these two games the Battle/Hero game in chapter 3 . 


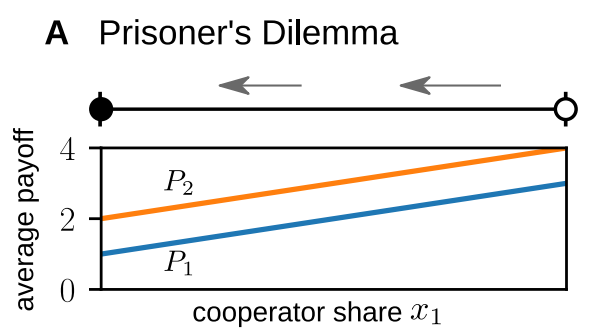

B Harmony
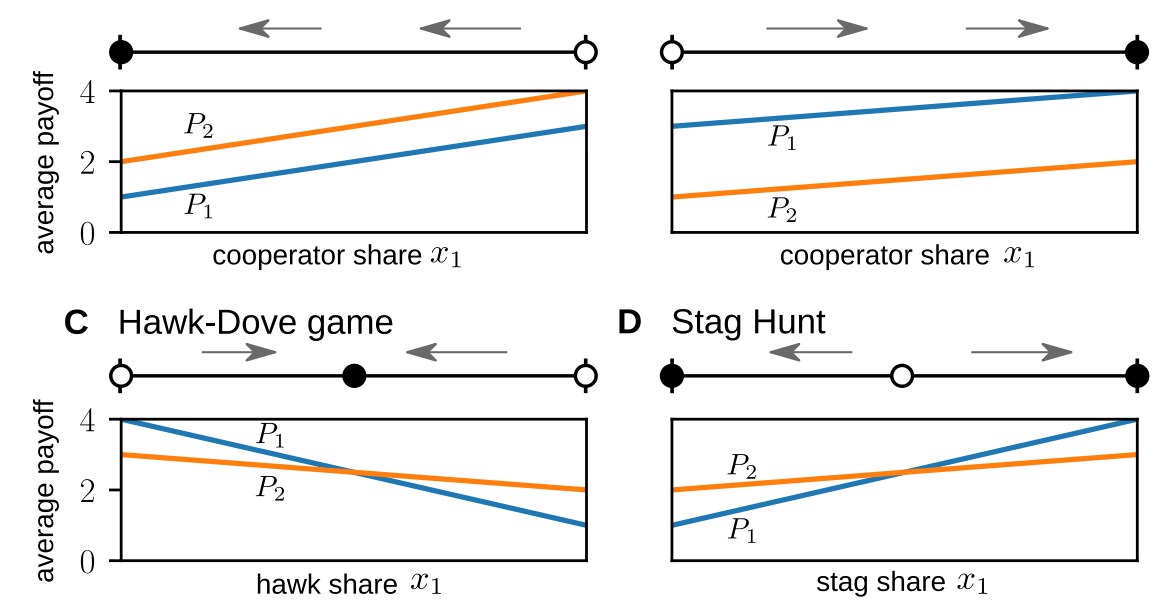

D Stag Hunt
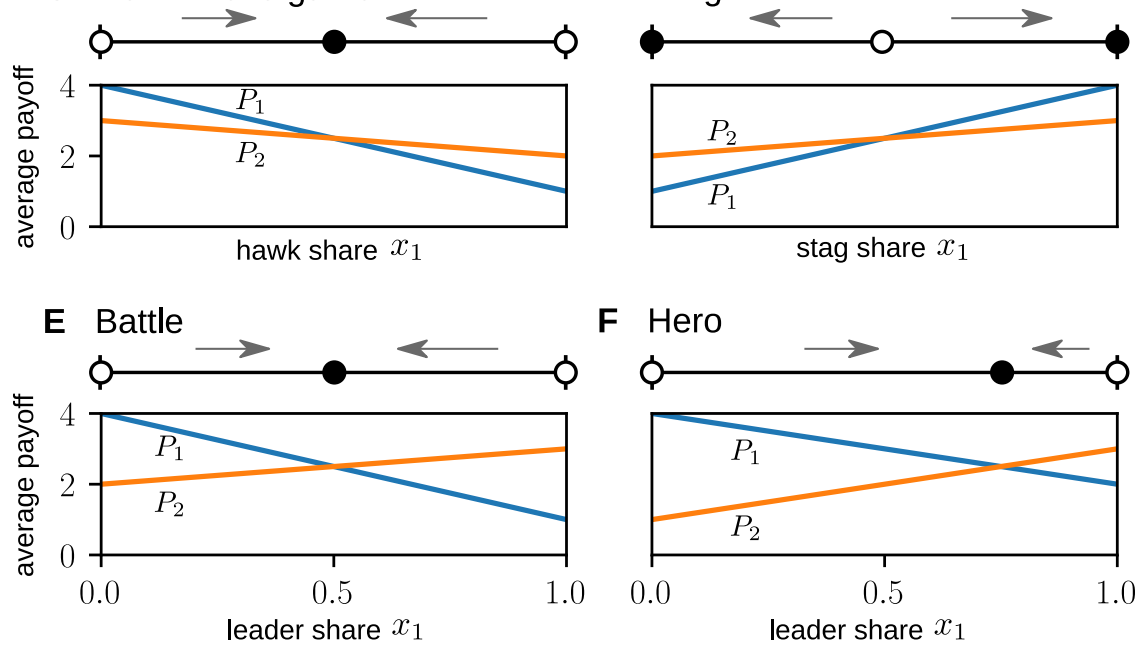

Figure 2.4: Illustrations of evolutionary games. The lines above the plots show the evolutionary dynamics with filled circles for stable fixed points and empty circles for unstable fixed points. The red and blue lines show the received average payoffs $P_{1}$ and $P_{2}$ resulting from the payoff matrices given in section 2.5, where strategy 1 is the cooperator strategy in (A) and (B), the hawk strategy in (C), the stag strategy in (D) and the leader strategy in (E) and (F). 


\section{EVOLUTIONARY GAME THEORY WITH PAYOFF FLUCTUATIONS}

\subsection{INTRODUCTION}

How species interact depends on the environment and is thus often uncertain or subject to ongoing variations. Traditional evolutionary game theory has assumed constant payoff structures. Here, we demonstrate by independent methods that the dynamics of averaged payoff values does not well approximate the dynamics of fluctuating payoff values. We show that payoff fluctuations induce qualitative changes in the dynamics. For instance, a Prisoner's Dilemma with payoff fluctuations may have the evolutionary dynamics of a HawkDove game with constant payoff values. As a consequence, cooperators can coexist with defectors - without any further cooperation maintaining mechanism such as kin or group selection [60, 61], reciprocity [62], or spatial structures [12].

First of all, how environmental fluctuations and payoff stochasticities affect the evolution of interacting species depends on the time scales. If the fluctuations are much faster than reproduction, adaptation reaches a stationary state where species are adapted to living in a rapidly fluctuating environment. If the fluctuations are much slower than the generation time (e.g. ice ages or geomagnetic field reversals), adaptation quickly reaches a stationary state which slowly drifts to follow the fluctuation. Ultimately challenging is the case when the fluctuations and reproduction are at a similar pace such that adaptation is continuously following the environmental changes. Here, we show that such states are subject to noise-induced transitions. Noiseinduced transitions have been studied in dynamical systems, where the most prominent models study the effects of additive noise [4446]. In dynamical systems, both additive and multiplicative noise can lead to an array of anomalous noise-induced effects such as stochastic resonance [63] and the creation of stable states [47, 48]. We wish to investigate the consequences of multiplicative noise in evolutionary game theory that have not been systematically studied yet.

A number of studies used stochastic models of population extinction to analyze the impact of environmental stochasticity on the extinction risk of small and large populations [29-31]. Particular atten-
The content of this chapter is published in Physical Review Letters [59] (C) [2018] American Physical Society, reprint permitted by APS Copyright Policies), reprinted here in a slightly modified and rearranged form. 
tion has been spent on how the species' mean time to extinction depends on a small randomly varying growth rate [32], and on the autocorrelation of the environmental noise [33-41]. Likewise in evolutionary game theory, the question of how fixation, i. e. the transition to the survival of only one species, depends on environmental stochasticity attracted a lot of attention $[3,5,8,9,27,42]$. Recently, how the fixation depends on environmental stochasticity was also studied in the case of multi-player games [43].

As opposed to these efforts, we will focus on the impact of payoff fluctuations on the stationary states.

Environmental fluctuations have been integrated in models for evolutionary games in different ways, including fluctuating reproduction rates [22-25], selection strength [8] and population size [10, 11, 26-28]. We integrate environmental fluctuations as varying payoff values to study situations in which the environmental fluctuations affect the way the species interact. Thereby we assume that all individuals experience the same environment, meaning that the payoff values vary with time but not between individuals. Payoff noise has been studied in structured populations $[17,64]$ and in finite populations [9]. Opposed to these, we study payoff noise in unstructured populations and aim to understand how the evolutionary dynamics change.

We explore the landscape of dynamical changes of evolutionary games induced by such fluctuating payoffs. We consider both deterministic (e. g. seasonal) as well as stochastic fluctuations with varying intensities and correlations. For a realistic description it is necessary to also include intrinsic noise in finite populations $[2,3,5,30]$. However, we aim to reveal phenomena that were unknown so far because they were hidden by the idealized assumption of constant payoffs. Therefore we isolate the effects of fluctuating payoffs from the diverse effects of intrinsic noise in finite populations by studying the replicator equation, which describes the evolution of strategies in infinite populations, and the Moran process [55] for finite but large populations.

\subsection{ANOMALOUS EVOLUTIONARILY STABLE STATES}

Multiplicative growth is a common model that underlies both population and evolutionary dynamics. In the simple case of time-discrete exponential growth, the population number $n$ is described by $n_{t+1}=$ $r n_{t}$. Depending on the growth rate $r$, the population will diverge $(r>1)$, remain constant $(r=1)$ or decay $(0 \leq r<1)$. However, a time-dependent growth rate $r_{t}$ can lead to intricate results. As an example, compare a growth rate that is switching between 1 and 1.1 with a growth rate that is switching between 0.6 and 1.5. Both have the same arithmetic average that is greater than one, but the population will diverge in the first case because $1 \cdot 1.1=1.1$ and decay in the 


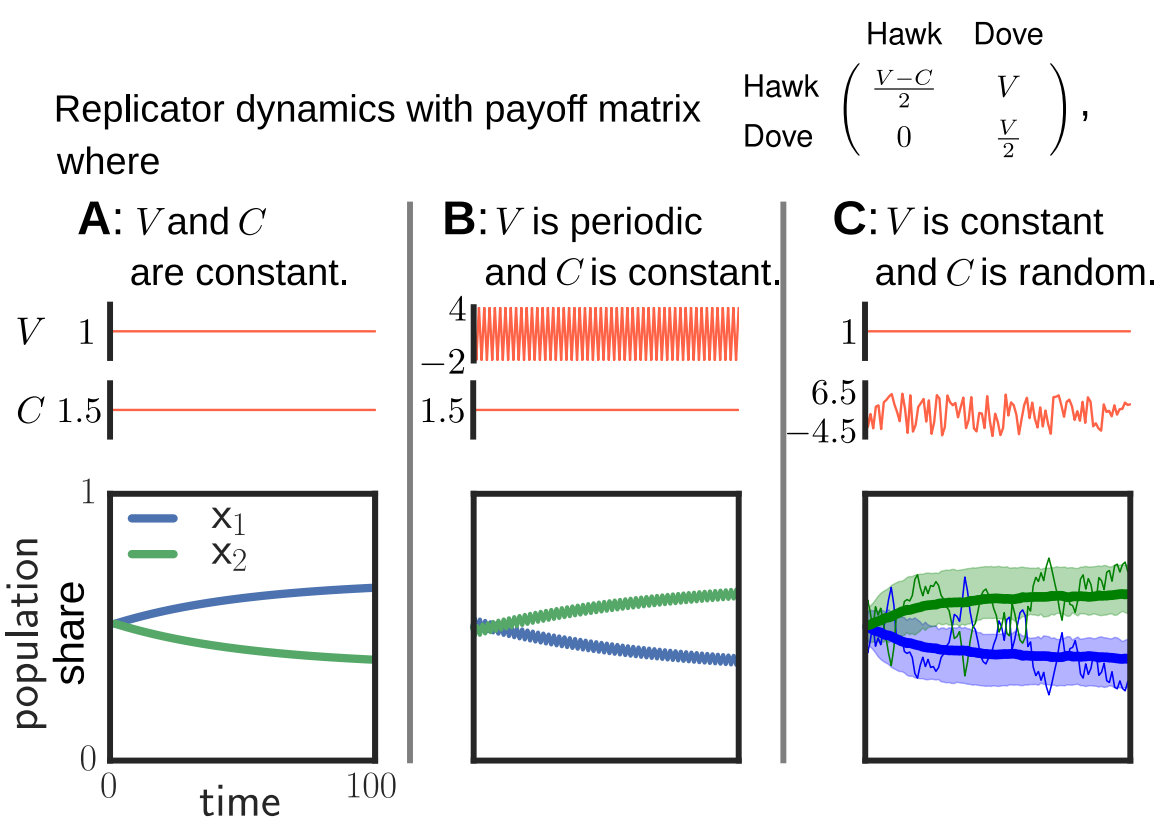

Figure 3.1: Selection reversal in a Hawk-Dove game with constant, periodic and random payoff. (A) describes a traditional Hawk-Dove game. The population starts at $x_{1}=x_{2}=0.5$ (50 \% hawks, $50 \%$ doves) and converges to an evolutionarily stable state where $x_{1}>x_{2}$. Periodically (B) or randomly fluctuating payoffs (C) shift the evolutionarily stable state such that $x_{1}<x_{2}$.

second case because $0.6 \cdot 1.5=0.9$. In general, the long-term growth is determined by the geometric mean of the growth rate $\bar{r}$, and the population will diverge if $\bar{r}>1$, remain constant if $\bar{r}=1$ and decay if $0 \leq \bar{r}<1$. Like in this example, multiplicative noise has generally a net-negative effect on growth in the long-term [65-67].

Models of evolutionary game theory are more complex but share the same underlying property, which leads to noise-induced non-ergodic behavior.

In the classical Hawk-Dove game two birds meet and compete for a shareable resource $V$, the positive payoff. If a hawk meets a dove the hawk alone gets the resource, if two doves meet they share the resource and if two hawks meet they fight for the resource, which costs energy and implies the risk of getting injured, formalized by a negative payoff $-C$. Since $50 \%$ of the hawks win and $50 \%$ of the hawks loose a fight, the average payoff of a hawk meeting a hawk in the limit of an infinite population is $\frac{V-0}{2}+\frac{0-C}{2}=\frac{V-C}{2}$.

Figure 3.1A shows that for $V=1$ and $C=1.5$ the time-discrete replicator dynamics leads to an evolutionarily stable state in which a larger population of hawks coexists with a smaller population of doves. However, in a changing environment the payoff matrix will not be constant. For example, the abundance of the food resource may change periodically with the seasons, or the risk of death caused by an injury may depend on the presence of predators. Figure 3.IB 

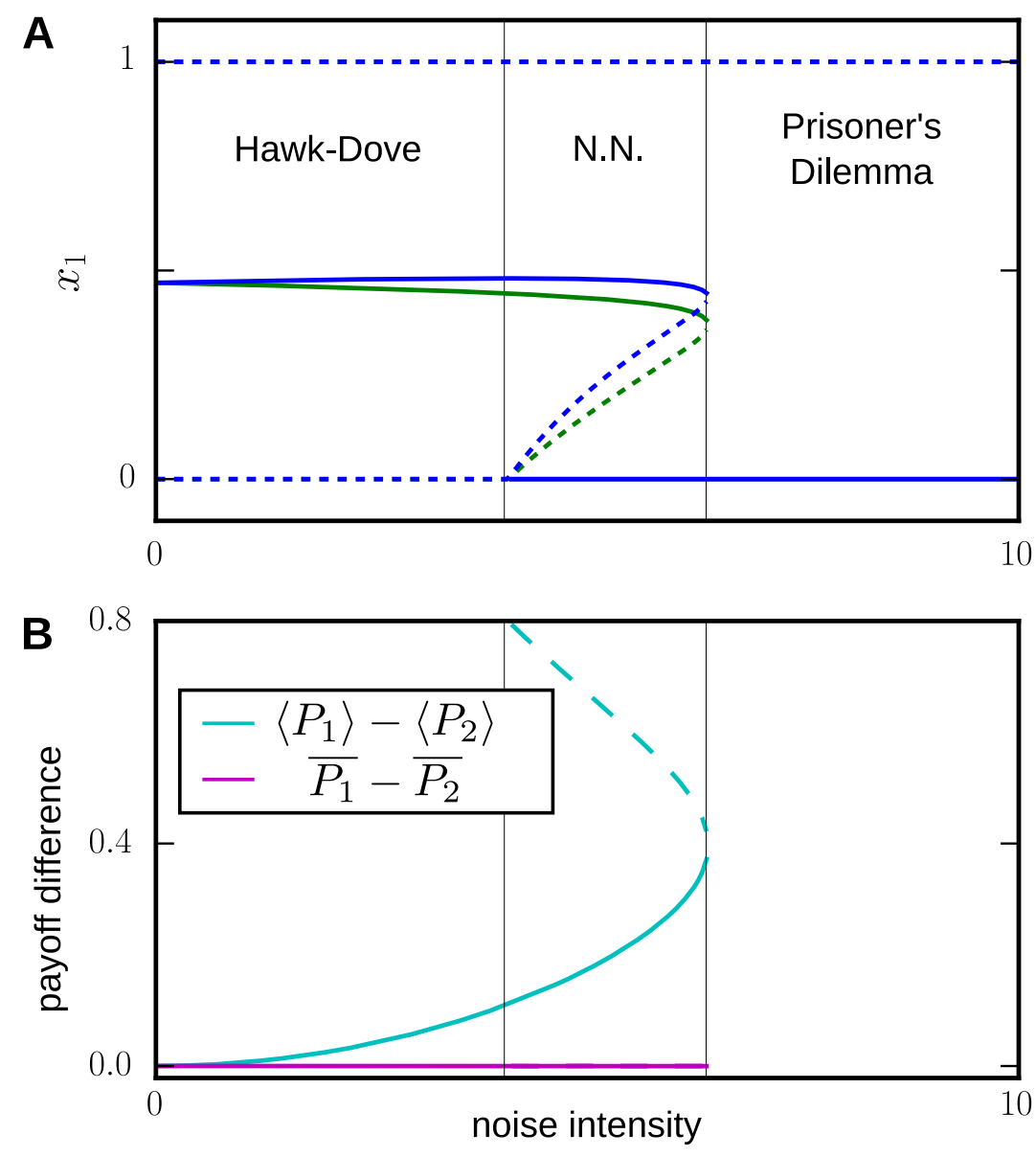

Figure 3.2: Fluctuations transform a Hawk-Dove game into a Prisoner's Dilemma and cause "unfair" stable coexistence. (A) Shown is the anomalous stationary state (solid line: stable, dashed line: unstable) of the fraction of cooperators $x_{1}$ as a function of the noise intensity. Due to alternating payoff values the stationary states consist of two periodic points (green and blue). With increasing intensity, the dynamical structure of a Hawk-Dove game first changes to a game without analog in traditional games (N.N.) and finally to a Prisoner's Dilemma game. (B) The difference of the averaged payoffs received by the two players corresponding to the stationary states of coexistence in $(\mathbf{A})$. In the arithmetic mean the received payoffs are unfair. In the geometric mean they are equal, as predicted by equation 3.4. 
and 3.1C show how the evolutionarily stable state can change if $V$ or $C$ fluctuate such that their averages are still the same as in figure 3.1A. Similar to the aforementioned example with the exponential growth process, the noise has a net-negative effect on the long-term growth of the strategies in replicator dynamics, too. Due to the specific structure of the Hawk-Dove game payoff matrix, the negative effect of the noise of both $V$ and $C$ is stronger for the population of hawks than for the doves, such that with sufficient noise the doves dominate the population in the evolutionarily stationary state. Next, we show that these anomalous effects are generic for evolutionary games.

In evolutionary game theory the interactions are usually formalized in a payoff function, which specifies the reward from the interaction with another player that is received by a given individual. In the simplest case, a game with two strategies is determined by a payoff matrix $M$ with $2 \times 2$ matrix elements. We describe the state of the population as $\mathbf{x}\left(\sum x_{i}=1\right)$, where $x_{i} \geq 0$ is the fraction of players with strategy $i \in\{1,2\}$. Players with strategy $i$ receive the payoff $P_{i}=(M \mathbf{x})_{i}+b$, where the background fitness $b$ ensures that the payoff is positive. The assumption that species that receive a higher payoff reproduce faster can be formalized by the replicator equation, which is used here in the time-discrete form [68]

$$
\begin{gathered}
x_{i}^{(t+1)}=x_{i}^{(t)} \cdot r_{i}\left(\mathbf{x}^{(t)}, M\right), \\
\text { with } r_{i}\left(\mathbf{x}^{(t)}, M\right)=\frac{\left(M \mathbf{x}^{(t)}\right)_{i}+b}{\mathbf{x}^{(t) T} M \mathbf{x}^{(t)}+b}=\frac{P_{i}}{\langle P\rangle}
\end{gathered}
$$

and the average payoff of the population $\langle P\rangle=x_{1} P_{1}+x_{2} P_{2}$.

Following Smith [69], "a population is said to be in an 'evolutionarily stable state' [henceforth ESS] if its genetic composition is restored by selection after a disturbance, provided the disturbance is not too large." Hence the ESS describe the long-term behavior of the system and are stable stationary states of equation 3.1. For a constant payoff matrix $M$, the stationary states $\mathbf{x}^{*}$ satisfy $r_{i}\left(\mathbf{x}^{*}, M\right)=1$. If two species coexist, $r_{1}\left(\mathbf{x}^{*}, M\right)=r_{2}\left(\mathbf{x}^{*}, M\right)$ implies that both receive the same payoff $P_{1}=P_{2}=\langle P\rangle$, as otherwise the species with the higher payoff would move the system away from this state due to faster growth.

Now consider continuously changing payoffs with finite means. The stationary states $\mathbf{x}^{*}(t)$ are solutions of

$$
\overline{r_{i}\left(\mathbf{x}^{*}, M\right)}:=\lim _{T \rightarrow \infty}\left(\prod_{t=0}^{T-1} r_{i}\left(\mathbf{x}^{*}(t), M^{(t)}\right)\right)^{\frac{1}{T}}=1,
$$

where $M^{(t)}$ is the time-dependent payoff matrix. Equation 3.3 defines the geometric average, indicated henceforth by the bar. If the payoff matrix changes deterministically with period $T$ a stationary state is a periodic function $\mathbf{x}^{*}(t)=\mathbf{x}^{*}(t+T)$; if it changes randomly a 
stationary state is a random function $\mathbf{x}^{*}(t)$ with distribution $\rho^{*}(\mathbf{x})$. But how does one calculate the stationary states for periodically and randomly changing payoff matrices? In contrast to normal ESS the stationary states are not solutions of $\left\langle P_{1}\right\rangle=\left\langle P_{2}\right\rangle$, where $\left\langle P_{i}\right\rangle:=$ $\lim _{T \rightarrow \infty} \frac{1}{T} \sum_{t=0}^{T-1}\left(M^{(t)} \mathbf{x}^{*}(t)\right)_{i}$ is the arithmetic time average of the received payoff. Equation 3.3 implies that $\overline{r_{1}\left(\mathbf{x}^{*}, M\right)}=\overline{r_{2}\left(\mathbf{x}^{*}, M\right)}=1$, and, using equation 3.2 , that

$$
\overline{P_{1}}=\overline{P_{2}} \text {. }
$$

If the fluctuations are small, we can approximate the geometric mean by $\overline{P_{i}}=\left\langle P_{i}\right\rangle-\frac{\sigma_{i}^{2}}{2\left\langle P_{i}\right\rangle}+\mathcal{O}\left(\sigma_{i}^{4}\right)$ (see section 3.6.1), where $\sigma_{i}^{2}=\operatorname{Var}\left[P_{i}\right]$. Using this approximation in equation 3.4 yields

$$
\left\langle P_{1}\right\rangle-\frac{\sigma_{1}^{2}}{2\left\langle P_{1}\right\rangle}=\left\langle P_{2}\right\rangle-\frac{\sigma_{2}^{2}}{2\left\langle P_{2}\right\rangle}
$$

Equation 3.5 shows that $\left\langle P_{1}\right\rangle$ and $\left\langle P_{2}\right\rangle$ are generally different, which is why we call these stationary states unfair. It includes the case of constant payoff values as a special case ${ }^{1}$. Figure 3.2A illustrates how payoff fluctuations may change the evolutionary dynamics and thereby transform one game into another game. Figure 3.2B shows how the arithmetic and the geometric average of the payoffs the two species receive deviate. Figure 3.3 shows the stationary states and the corresponding received payoffs of both species for constant and varying payoff values.

\section{$3 \cdot 3$ DETERMINISTIC PAYOFF FLUCTUATIONS}

We first consider deterministic payoff fluctuations under the replicator equation (equation 3.1). To find the stationary state $\mathbf{x}^{*}$ we solve equation 3.3. We assume that $M^{(t)}$ is a sequence with period T. Consequently, the stationary state $\mathbf{x}^{*(t)}$ is periodic as well and $P(\mathbf{x}, M)=$ $\frac{1}{T} \sum_{t=0}^{T} \delta\left(\mathbf{x}-\mathbf{x}^{*(t)}\right) \delta\left(M-M^{(t)}\right)$. Equation $3 \cdot 3$ reduces to

$$
\overline{r_{i}\left(\mathbf{x}^{*}, M\right)}=\left(\prod_{t=t^{\prime}}^{t^{\prime}+T} r_{i}\left(\mathbf{x}^{*(t)}, M^{(t)}\right)\right)^{\frac{1}{T}}=1 .
$$

Note that equation 3.6 has only one free variable because if one periodic point $\mathbf{x}^{*\left(t^{\prime}\right)}$ is given, the others are determined by equation 3.1. As an illustrative example, assume an alternating payoff matrix $M^{(t)}=$

1 Note that $\sigma_{1}$ and $\sigma_{2}$ depend on the stationary state $x_{1}$ and the variance and covariance of the payoff values $M=\left[m_{1}, m_{2}, m_{3}, m_{4}\right]$. If $\sigma_{1}=\sigma_{2}=0$, equation 3.5 reduces to $\left\langle P_{1}\right\rangle=\left\langle P_{2}\right\rangle$. For small fluctuations we can approximate them as $\sigma_{1}^{2} \approx \mathrm{E}\left[x_{1}\right]^{2} \operatorname{Var}\left[m_{1}\right]+\left(1-\mathrm{E}\left[x_{1}\right]\right)^{2} \operatorname{Var}\left[m_{2}\right]+2\left(\mathrm{E}\left[x_{1}\right]-\mathrm{E}\left[x_{1}\right]^{2}\right) \operatorname{Cov}\left[m_{1}, m_{2}\right]$ and $\sigma_{2}^{2} \approx \mathrm{E}\left[x_{1}\right]^{2} \operatorname{Var}\left[m_{3}\right]+\left(1-\mathrm{E}\left[x_{1}\right]\right)^{2} \operatorname{Var}\left[m_{4}\right]+2\left(\mathrm{E}\left[x_{1}\right]-\mathrm{E}\left[x_{1}\right]^{2}\right) \operatorname{Cov}\left[m_{3}, m_{4}\right]$. 
constant payoff
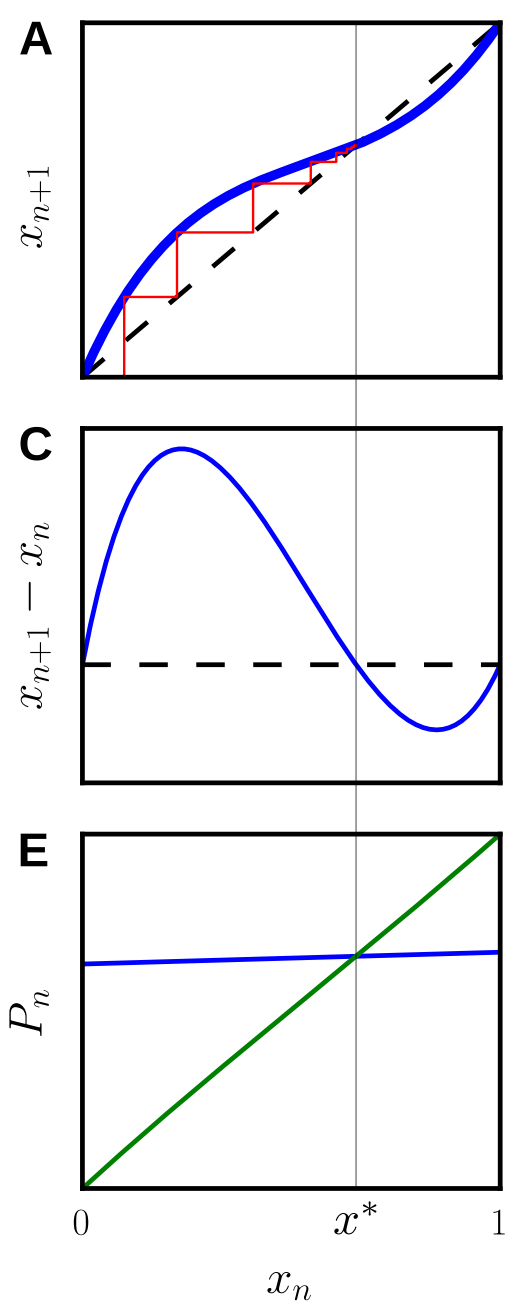

varying payoff
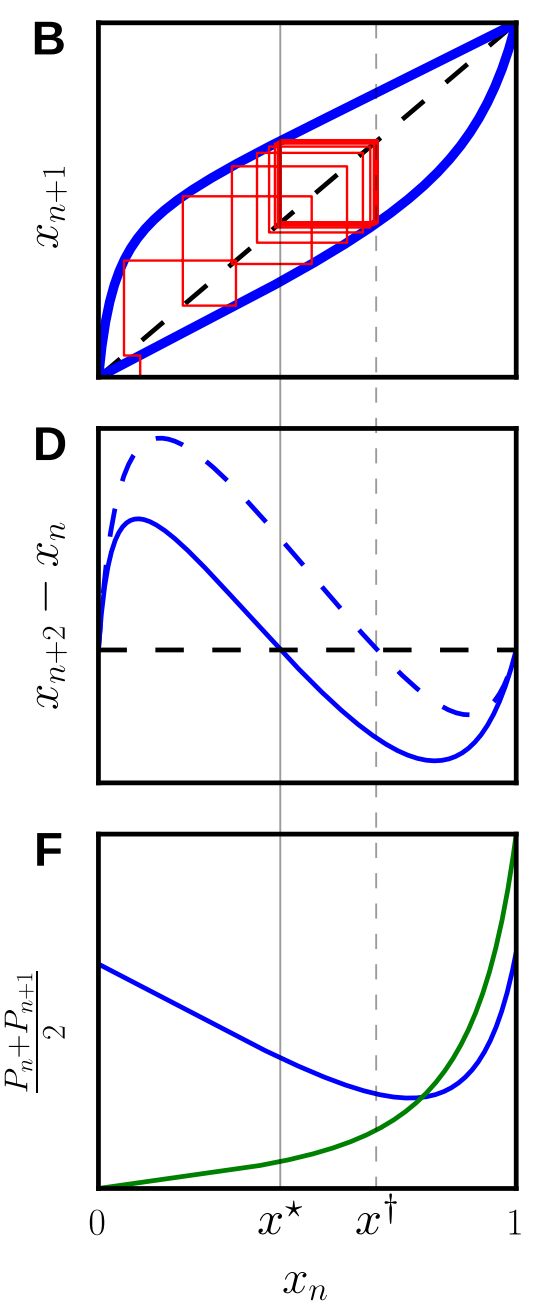

Figure 3.3: Evolutionarily stable states for constant and periodic payoff. An evolutionary game with a time-constant payoff matrix (left) compared with a game with a time-varying payoff matrix (right) to exemplify the "unfair" stationary states. The former payoff matrix represents a Hawk-Dove game and the latter an alternating payoff matrix which has the same time-average as the constant Hawk-Dove game. (A) and (B) show the dynamics of the two games as a Verhulst diagram with an example trajectory in red. The two blue curves in (B) correspond to even and odd time points, with the anomalous stationary states at $x_{n}^{\star}$ and $x_{n}^{\dagger}$. (C) and (D) show the difference of the state one (C) or two (D) time steps later and the current state. The zero crossings of these lines are the positions of the fixed point and the periodic points. (E) and (F) show the payoff of species 1 (blue) and species 2 (green). In (E), the equilibrium is at the same position as the fixed point. In (F), species 1 receives a higher time-averaged payoff than species 2 at both periodic points. 
$\bar{M}+(-1)^{t} \sigma \tilde{M}$. Then $\mathbf{x}^{*(t)}=\overline{\mathbf{x}}^{*}+(-1)^{t} \Delta \mathbf{x}^{*}$ has the same form and can be found by solving equation 3.6 , which reduces to

$$
\overline{r_{i}\left(\mathbf{x}^{*}, M\right)}=\sqrt{r_{i}\left(\mathbf{x}^{*(t)}, M^{(t)}\right) \cdot r_{i}\left(\mathbf{x}^{*(t+1)}, M^{(t+1)}\right)}=1 \text {. }
$$

Figure 3.2 shows the stationary states of a game with the payoff function

$$
M^{(t)}=\left(\begin{array}{cc}
1.1 & 0.8 \\
2 & 0
\end{array}\right)+(-1)^{t} \sigma\left(\begin{array}{cc}
-0.33 & 1 \\
1 & 0
\end{array}\right)
$$

For $\sigma=0$ this is a Hawk-Dove game. For small $\sigma$, in fact, the stationary states predicted by equation 3.7 slightly deviate from the ESS of the Hawk-Dove game. There is a first bifurcation at $\sigma \approx 4.07$, from one stable stationary state (solid curves) to two. At $\sigma \approx 6.4$ there is a second bifurcation where the first branch, the stable coexistence, disappears. The bifurcation behavior induces a pronounced hysteresis effect. Ergodicity breaking causes anomalous player's payoff expectations as shown in figure $3.2 \mathrm{~B}$. The arithmetic mean of the payoff difference that the players receive also shows a pronounced hysteresis effect. For the geometric mean, as predicted by equation 3.4, this effect is absent.

More generally, fluctuations can even change the number, the positions and the stability of stationary states and the dynamics can be structurally very different from the dynamics of games with constant payoffs, as shown in figure 3.4. In figure 3.4A large fluctuations induce the onset of cooperation for the Prisoner's dilemma as it is effectively transformed to a Hawk-Dove game with stable coexistence. Figures $3.4 \mathrm{~B}, 3.4 \mathrm{C}$ and $3.4 \mathrm{D}$ show how increasing fluctuations successively transform three other classical games either into different classical games or into games without classical analogs (denoted at “N.N.").

In section 3.6.2 we show how anomalous stationary states arise from (correlated) stochastic payoffs, which is mathematically more involving but shows similar effects as from deterministic fluctuations.

\subsection{CLASSIFICATION OF GAMES WITH PAYOFF FLUCTUATIONS}

A symmetric game defined by a constant $2 \times 2$ payoff matrix can be classified as one out of 12 game classes with distinct dynamical structures, e. g. Prisoner's Dilemma, Hawk-Dove game, etc. This traditional classification (introduced in section 2.5) is based on the rank of the four values in the payoff matrix. For a complete list of the ranks of all 12 games see middle column in table 3.1.

The name of a game allows a more intuitive understanding than the position in the four dimensional payoff space. However, this classifi- 


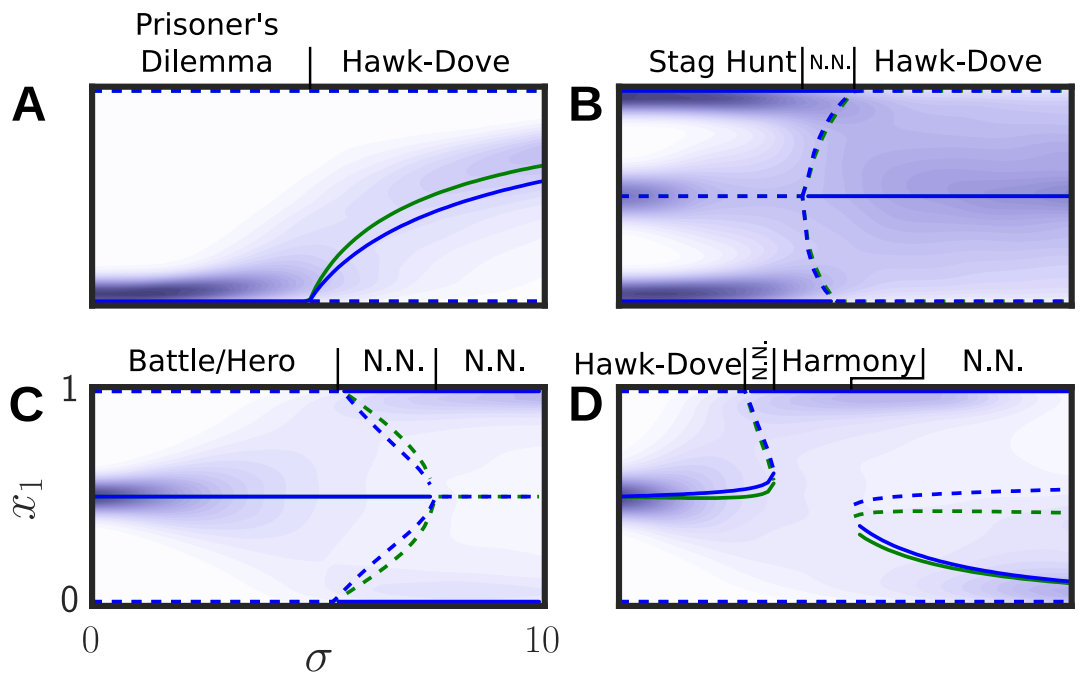

Figure 3.4: Evolutionarily stable states with increasing fluctuation intensity. Stable and unstable states (solid and dashed lines) $x_{1}^{*}(\sigma)$ for games with alternating payoff fluctuations (blue and green are the two periodic points). The payoff matrices are $M^{(t)}=[3,1,4,2]+(-1)^{t} \sigma[0,0,0,1]$ in $(\mathbf{A}), M^{(t)}=[4,1,3,2]+$ $(-1)^{t} \sigma[1,0,0,1]$ in $(\mathbf{B}), M^{(t)}=[2,3,4,1]+(-1)^{t} \sigma[0,1.3,1.3,0]$ in (C) and $M^{(t)}=[3,2,4,1]+(-1)^{t} \sigma[-0.75,1,-2,1]$ in (D). In each example the background fitness is $b=10$. The names of the games are identified using criteria described in section 3.4.

For the same games but stochastic instead of alternating noise, the background shows the average of three stationary distributions resulting from the initial distributions $\delta(x), \delta(x-0.5)$ and $\delta(x-1)$. 
cation cannot be applied to time-varying payoff matrices because the ranks may be time-dependent as well. Therefore we propose a classification for evolutionary games based on three characteristics: (1) the dynamics of the evolutionary game (the number of stationary states and their stability), (2) the type of social interaction (how the payoff differs between stationary states for one player compared to the other player) and (3) the effect on the community (how the total payoff of player one and two differs between stationary states). The classification scheme and its criteria are summarized in figure 3.5. Based on these criteria a game class is defined as a tuple $\left[c_{1}, c_{2}, c_{3}, c_{4}\right]$, where

$$
\begin{aligned}
& c_{1}=\operatorname{Sign}\left(\frac{\mathrm{d} u_{1}}{\mathrm{~d} x_{1}}(0)\right) \cdot n^{*} \\
& c_{2}=\operatorname{Sign}\left(P_{1}(1)-P_{1}(0)\right) \\
& c_{3}=\operatorname{Sign}\left(P_{2}(1)-P_{2}(0)\right) \\
& c_{4}=\operatorname{Sign}(\langle P\rangle(1)-\langle P\rangle(0)),
\end{aligned}
$$

where $u_{1}=\frac{\mathrm{d} x_{1}}{\mathrm{~d} t}, P_{i}(x)=\left(M\left(\begin{array}{c}x \\ 1-x\end{array}\right)\right)_{i}$ denotes the payoff of a strategy $i$ player, $\langle P\rangle=x P_{1}+(1-x) P_{2}$ the average payoff in the population and $n^{*}=\left\|\left\{x_{1}^{*}: u_{1}\left(x_{1}^{*}\right)=0\right\}\right\|$ the number of stationary states. This classification can be applied to games with varying payoff matrices and even games with nonlinear payoff functions. The scheme is developed for time-continuous dynamics. The formulation for timediscrete dynamics is analogous. Note also that the criteria $\left(c_{2}-c_{4}\right)$ of equations 3.9 can be written in a more general form to describe also non-monotonic payoff functions.

Table 3.1 lists the 12 traditional games defined by the payoff rank criteria and their corresponding definitions with the presented generalized criteria (for a proof see section 3.6.3).

As an illustrative example how to apply the generalized criteria we show that the game in figure 3.2 at noise intensity 8 , where the payoff matrix is

$$
M^{(t)}=\left(\begin{array}{cc}
1.1 & 0.8 \\
2 & 0
\end{array}\right)+8 \cdot(-1)^{t}\left(\begin{array}{cc}
-0.33 & 1 \\
0 & 0
\end{array}\right)
$$

is a Prisoner's Dilemma.

As we can see in the figure there are two stationary states, a stable state at $x_{1}=0$ and an unstable state at $x_{1}=1$, consequently $c_{1}=-2$. From the expected payoff

$$
P_{i}\left(x_{1}\right)=\frac{1}{2}\left(M^{\text {even } \mathrm{t}}\left(\begin{array}{c}
x_{1} \\
1-x_{1}
\end{array}\right)+M^{\text {odd } \mathrm{t}}\left(\begin{array}{c}
x_{1} \\
1-x_{1}
\end{array}\right)\right)
$$


A Dynamics
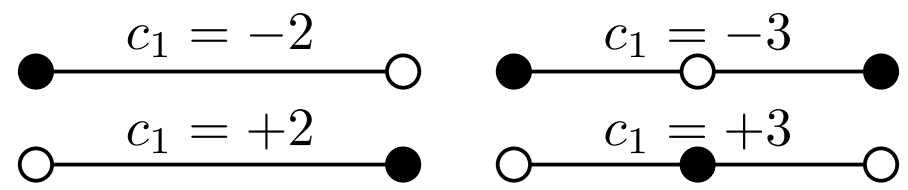

B Type of social interaction

\begin{tabular}{c|cc} 
& $c_{3}=+1$ & $c_{3}=-1$ \\
\hline$c_{2}=+1$ & $\begin{array}{l}\text { mutual } \\
\text { benefit }\end{array}$ & selfishness \\
$c_{2}=-1$ & altruism & spite
\end{tabular}

C Effect on community
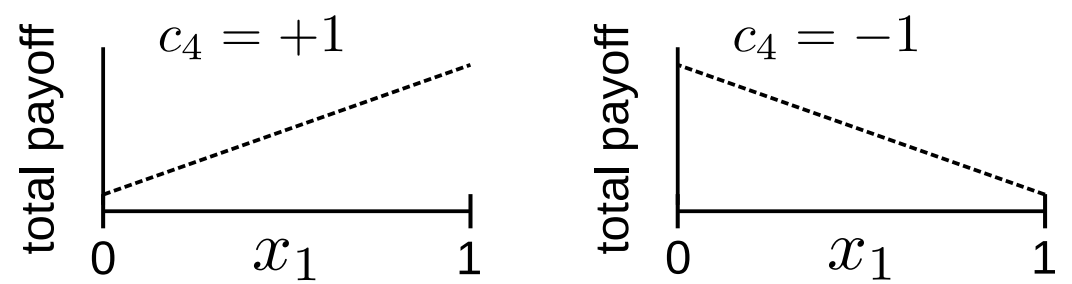

Figure 3.5: Case differentiation of game characteristics. An evolutionary game with two strategies and constant payoff has one out of (A) four possible dynamical structures (either two or three stationary states with the first one either stable or unstable), (B) four possible combinations of strategy $I^{\prime}$ s impact on the payoff of the individuals (positive or negative impact on the payoff of strategy 1 and 2 players), and (C) two possible kinds of strategy I's impact on the total payoff of all players (positive or negative).

evaluated at the stationary states $\left(P_{1}(0)=0.8, P_{1}(1)=1.1, P_{2}(0)=0\right.$ and $\left.P_{2}(1)=2\right)$ it follows that $c_{2}=+1$ and $c_{3}=+1$. For the last criteria we evaluate the population payoff $\langle P\rangle\left(x_{1}\right)=x_{1} P_{1}+\left(1-x_{1}\right) P_{2}$ at the stationary states $\left(\langle P\rangle(0)=P_{2}(0)\right.$ and $\left.\langle P\rangle(1)=P_{1}(1)\right)$, which results in $c_{4}=+1$. To summarize, the game satisfies the generalized criteria $[-2,+1,+1,+1]$. According to table 3.1 this defines a Prisoner's Dilemma.

The games in figure 3.4 were determined with this method. 


\begin{tabular}{|c|c|c|}
\hline Name & $\begin{array}{c}\text { Payoff rank } \\
\text { criteria }\end{array}$ & $\begin{array}{l}\text { Generalized } \\
\text { criteria }\end{array}$ \\
\hline \multirow{2}{*}{ Hawk-Dove } & {$[3,2,4,1]$} & {$[+3,+1,+1,+1]$} \\
\hline & {$[1,4,2,3]$} & {$[+3,-1,-1,-1]$} \\
\hline \multirow{2}{*}{ Battle } & {$[2,3,4,1]$} & \multirow{4}{*}{$\begin{array}{l}{[+3,-1,+1,+1]} \\
{[+3,-1,+1,-1]}\end{array}$} \\
\hline & {$[1,4,3,2]$} & \\
\hline \multirow{2}{*}{ Hero } & {$[1,3,4,2]$} & \\
\hline & {$[2,4,3,1]$} & \\
\hline \multirow{2}{*}{ Compromise } & {$[1,2,4,3]$} & {$[-2,-1,+1,-1]$} \\
\hline & {$[3,4,2,1]$} & {$[+2,-1,+1,+1]$} \\
\hline \multirow{2}{*}{ Deadlock } & {$[2,1,4,3]$} & {$[-2,+1,+1,-1]$} \\
\hline & {$[3,4,1,2]$} & {$[+2,-1,-1,+1]$} \\
\hline \multirow{2}{*}{ Prisoner's Dilemma } & {$[3,1,4,2]$} & {$[-2,+1,+1,+1]$} \\
\hline & {$[2,4,1,3]$} & {$[+2,-1,-1,-1]$} \\
\hline \multirow{2}{*}{ Stag Hunt } & {$[4,1,3,2]$} & {$[-3,+1,+1,+1]$} \\
\hline & {$[2,3,1,4]$} & {$[-3,-1,-1,-1]$} \\
\hline \multirow{2}{*}{ Assurance } & {$[4,1,2,3]$} & \multirow{4}{*}{$\begin{array}{l}{[-3,+1,-1,+1]} \\
{[-3,+1,-1,-1]}\end{array}$} \\
\hline & {$[3,2,1,4]$} & \\
\hline \multirow{2}{*}{ Coordination } & {$[4,2,1,3]$} & \\
\hline & {$[3,1,2,4]$} & \\
\hline \multirow{2}{*}{ Peace } & {$[4,3,1,2]$} & {$[+2,+1,-1,+1]$} \\
\hline & {$[2,1,3,4]$} & {$[-2,+1,-1,-1]$} \\
\hline \multirow{2}{*}{ Harmony } & {$[4,3,2,1]$} & \multirow{4}{*}{$\begin{array}{l}{[+2,+1,+1,+1]} \\
{[-2,-1,-1,-1]}\end{array}$} \\
\hline & {$[1,2,3,4]$} & \\
\hline \multirow{2}{*}{ Concord } & {$[4,2,3,1]$} & \\
\hline & {$[1,3,2,4]$} & \\
\hline
\end{tabular}

Table 3.1: Criteria for strict symmetric games. The middle column shows the rank of the values in the payoff matrix $M=[a, b, c, d]$, e.g. $[3,2,4,1]$ means $d<b<a<c$ [58]. The right column shows the values of the criteria defined in the text. Some games are two different games according to the payoff rank criteria and the same game according to the generalized criteria, because their evolutionary dynamics is structurally the same. For the example of the Battle and the Hero game, this structural similarity is explained in section 2.5 . 


\section{$3 \cdot 5$ Discussion}

Payoff noise in evolutionary dynamics is multiplicative and as such causes ergodicity breaking. The consequences have intricate effects on the coevolution of strategies. Depending on the details of the system, on the intensity of the fluctuations and even on their covariance, ergodicity breaking leads to shifting the payoffs out of equilibrium, shifting the stationary states and thereby to fundamental structural changes of the dynamics.

In evolutionary games with constant payoffs, the condition for stable coexistence is that all species have equal growth rates. With fluctuating payoffs this condition generalizes to equal time-averaged growth rates, which typically are different from ensemble averages in nonergodic systems. When one naively replaces fluctuating payoffs with their average values, the ensemble averages of the growth rates are recovered but these averages do not correctly predict the dynamics.

Games with fluctuating payoffs require a novel classification that cannot be based on payoff ranking schemes as introduced in section 2.5. We developed a classification that primarily considers the dynamical structure. Our classification for evolutionary games may be applied to evolutionary games where the payoff structure cannot be described by a simple payoff matrix, or when other modifications affect the dynamical structure. Examples include complex interactions of microbes such as cooperating and free-riding yeast cells, where the payoff is a nonlinear function of the densities [52].

Payoff fluctuations can cause two strategies that coexist in an evolutionarily stable state to receive different time-averaged payoffs. However, these "unfair" stable states are not mutationally stable. Mutations, in fact, would turn the "unfair" stable state into a meta-game, where the beneficiary aims to increase and the victim aims to escape the unfairness. Strategies of this meta-game could be tuning the adaptation or reproduction rate according to the environmental fluctuation [25]. Phenotypic plasticity [70] and bet-hedging [71] may reduce the necessity to adapt at all.

To conclude, caution is advised when predictions are based on averaged observables, in particular, averaged payoffs structures. Depending on the amplitude and covariance of the fluctuations, the evolutionary dynamics can be qualitatively different. 


\subsection{PROOFS AND METHODS}

\subsubsection{Approximation of the geometric mean}

Let $X$ be a random variable with $\mathrm{E}[X]=\mu, \mathrm{E}\left[(X-\mathrm{E}[X])^{2}\right]=\sigma^{2}$ and $\mathrm{E}\left[(X-\mathrm{E}[X])^{3}\right]=0$. We can write the geometric mean of $X$ as

$$
\bar{X}=\overline{\mu+\sigma Y}=\lim _{T \rightarrow \infty} \prod_{t=0}^{T}\left(\mu+\sigma y_{t}\right)^{\frac{1}{T}}
$$

where $Y$ is a random variable with $\mathrm{E}[Y]=0, \mathrm{E}\left[(Y-\mathrm{E}[Y])^{2}\right]=1$ and $\mathrm{E}\left[(Y-\mathrm{E}[Y])^{3}\right]=0$. Now we have the geometric mean as a function of $\sigma$ and can write the Taylor series of $\bar{X}(\sigma)$ at $\sigma=0$,

$$
\begin{aligned}
\bar{X} & =\bar{X}(0)+\frac{\mathrm{d} \bar{X}}{\mathrm{~d} \sigma}(0) \sigma+\frac{\mathrm{d}^{2} \bar{X}}{\mathrm{~d} \sigma^{2}}(0) \frac{\sigma^{2}}{2}+\frac{\mathrm{d}^{3} \bar{X}}{\mathrm{~d} \sigma^{3}}(0) \frac{\sigma^{3}}{6}+\mathcal{O}\left(\sigma^{4}\right) \\
& =\mu-\frac{\sigma^{2}}{2 \mu}+\mathcal{O}\left(\sigma^{4}\right)
\end{aligned}
$$

\subsubsection{Stochastic payoff fluctuations}

\subsubsection{Replicator equation}

How do anomalous stationary states arise from stochastic payoffs? To avoid unnecessary technicalities, we consider the case of two strategies, in which the state is fully described by a scalar $x=x_{1}$ (because $\left.x_{2}=1-x_{1}\right)$ and the payoff $M=\mathbf{Y}=\left(\begin{array}{ll}Y_{1} & Y_{2} \\ Y_{3} & Y_{4}\end{array}\right)$ is a random matrix, where $Y_{j}$ have probability density functions $P_{Y_{j}}\left(y_{j}\right)$, mean $E\left(Y_{j}\right)=\mu_{j}$ and variance $\operatorname{Var}\left(Y_{j}\right)=\sigma_{j}^{2}$. In short, we can write the replicator equation as

$$
X^{(t+1)}=f\left(X^{(t)}, \mathbf{Y}\right)
$$

with $f(x, \mathbf{y})=x^{(t)} \cdot \frac{\left(y^{(t)} \mathbf{x}^{(t)}\right)_{1}+b}{\mathbf{x}^{(t) T} \mathbf{y}^{(t)} \mathbf{x}^{(t)}+b}$. In order to get a function which is injective with respect to $\mathbf{Y}$ we define a new function

$$
f^{\prime}\left(X^{(t)}, \mathbf{Y}\right)=\left(\begin{array}{c}
f\left(X^{(t)}, \mathbf{Y}\right) \\
Y_{2} \\
Y_{3} \\
Y_{4}
\end{array}\right)
$$


This function is invertible, hence we can derive the joint probability $P_{f^{\prime}}\left(x^{(t+1)}, y_{2}, y_{3}, y_{4}\right)$ from the joint probability $P_{\mathbf{Y}}\left(y_{1}, y_{2}, y_{3}, y_{4}\right)=$ $P\left(y_{1}\right) P\left(y_{2}\right) P\left(y_{3}\right) P\left(y_{4}\right)$ by changing variables,

$$
P_{f^{\prime}}\left(x^{(t+1)}, y_{2}, y_{3}, y_{4}\right)=\left|\operatorname{det}\left[D f^{\prime-1}\right]\right| P_{\mathbf{Y}}\left(f^{\prime-1}\right)
$$

The stochastic kernel can be derived by marginalizing over $y_{2}, y_{3}$ and $y_{4}$.

$$
\begin{array}{r}
K\left(x^{(t+1)} \mid x^{(t)}\right)=\iiint P_{f^{\prime}}\left(x^{(t+1)}, y_{2}, y_{3}, y_{4}\right) \times \\
P_{Y_{2}}\left(y_{2}\right) P_{Y_{3}}\left(y_{3}\right) P_{Y_{4}}\left(y_{4}\right) \mathrm{d} y_{2} \mathrm{~d} y_{3} \mathrm{~d} y_{4}
\end{array}
$$

The Chapman-Kolmogorov equation gives the time evolution of the probability density

$$
P_{X}^{(t)}(x)=\int_{0}^{1} \mathrm{~d} x^{\prime} P_{X}^{(t-1)}\left(x^{\prime}\right) K\left(x \mid x^{\prime}\right),
$$

To ease the numerical evaluation we use the look-ahead-estimator [72]

$$
P_{X}^{(t)}(x)=\frac{1}{n} \sum_{l=1}^{n} K\left(x \mid s_{l}^{t-1}\right)
$$

where $\left\{s_{l}\right\}$ is a sample of size $n$ drawn from $P_{X}^{(t-1)}(x)$. Starting with an arbitrary initial distribution $P_{X}^{(1)}(x)$ and successively applying equation 3.20 converges to a stationary distribution $\rho^{*}(x)=P_{X}^{(\infty)}(x)$ of the stochastically driven replicator dynamics.

The background in figure 3.4 shows that the stationary distributions, apart from the expected broadening, follow the behavior of the stable states derived for analogous deterministic fluctuations.

We now demonstrate that the type of the distribution has only little effect on the stationary states. As an example we use a game with the payoff function

$$
M^{(t)}=\left(\begin{array}{cc}
1 & 0.5 \\
2 & 0
\end{array}\right)+X\left(\begin{array}{ll}
0 & 0 \\
1 & 0
\end{array}\right)
$$

with the background fitness $b=10$. Note that the zero-noise case of this game resembles a Hawk-Dove game. Figure 3.6 shows the stationary distributions $\rho^{*}\left(x_{1}\right)$ of the replicator dynamics and the Moran process, where $X$ is either a uniform, discrete, normal distributed random variable or alternations, each with variance $\sigma=2$. The higher moments of the noise distribution have little effect on the resulting stationary distribution. 

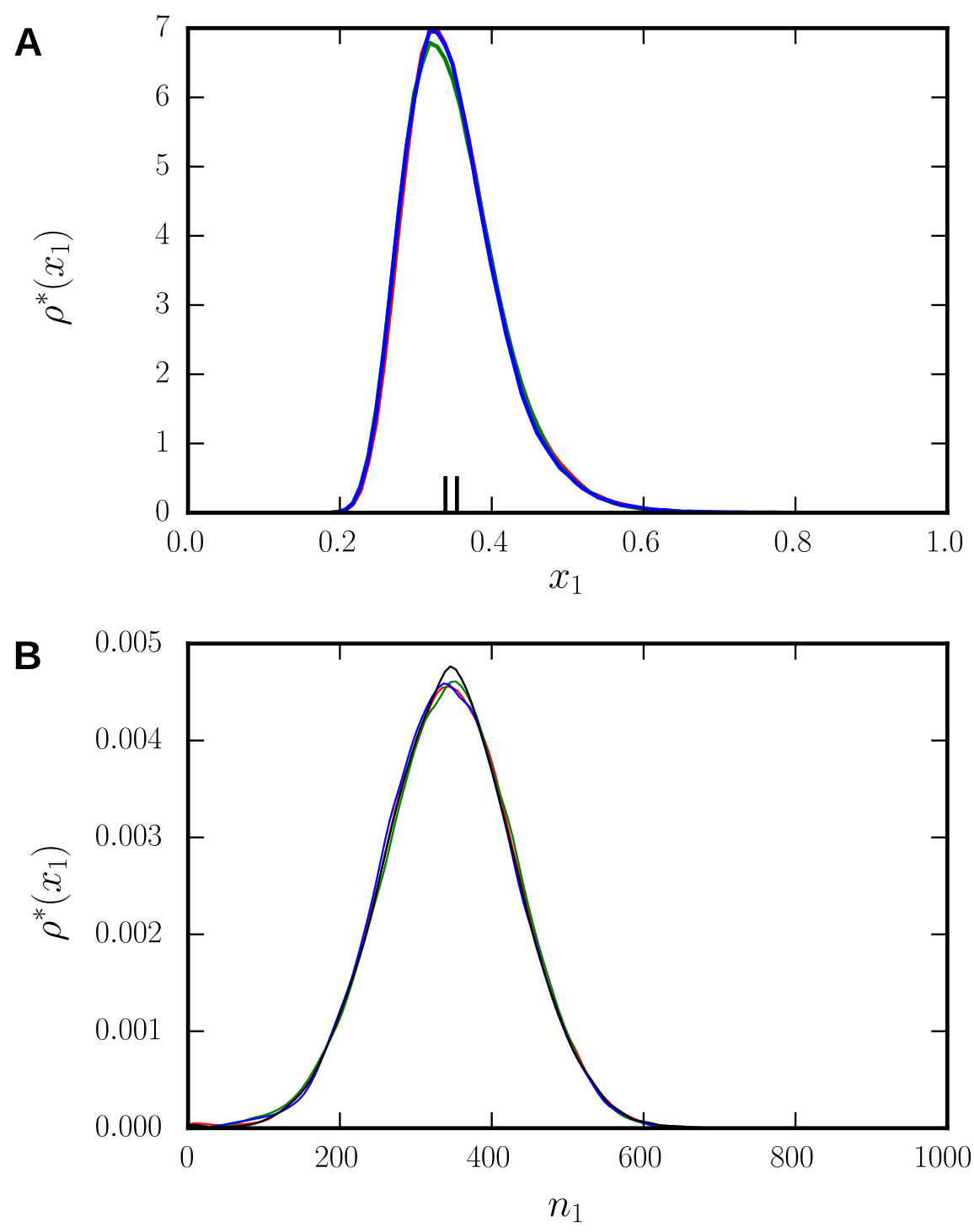

Figure 3.6: Stationary distributions with different types of noise. Stationary distributions obtained from replicator dynamics (A) and Moran model (B) for different noise sources with $\sigma=2$. Red: Uniformly distributed noise, green: discretely distributed noise, blue: normal distributed noise, black: alternating noise. 


\subsubsection{Moran processes}

Employment of Moran processes has been shown to be imperative for the mathematical understanding of stochastic evolutionary game theory. Despite being conceptionally very different from replicator dynamics, Moran processes are affected by payoff fluctuations in a similar way.

Consider a Moran process with population size $N$ and payoff matrix $M=\mathbf{Y}=\left(\begin{array}{ll}Y_{1} & Y_{2} \\ Y_{3} & Y_{4}\end{array}\right)$, where $Y_{j}$ are uncorrelated random variables with probability density functions $P_{Y_{j}}\left(y_{j}\right)$ (note that also a deterministically changing payoff with period $T=2$ can be mapped to this formulation $^{2}$ ). If the number of individuals playing strategy 1 is $i$, the expected payoff received by an individual playing strategy 1 or 2 is

$$
\begin{aligned}
& p_{1}(t)=\frac{1}{N-1}\left[y_{1}(t)(i-1)+y_{2}(t)(N-i)\right] \\
& p_{2}(t)=\frac{1}{N-1}\left[y_{3}(t) i+y_{4}(N-i-1)\right]
\end{aligned}
$$

With selection strength $w$ the fitness of each strategy $k=1,2$ reads

$$
f_{k}(t)=1-w+w p_{k} .
$$

The (non-zero) transition probabilities are

$$
\begin{aligned}
T(i \mid i+1)= & \int \frac{f_{1}(\mathbf{Y}) i(N-i)}{\left[f_{1}(\mathbf{Y}) i+f_{2}(\mathbf{Y})(N-i)\right] N} P_{\mathbf{Y}}(\mathbf{y}) \mathrm{d} \mathbf{y} \\
& +g(i / N) \\
T(i \mid i-1)= & \int \frac{f_{2}(\mathbf{Y}) i(N-i)}{\left[f_{1}(\mathbf{Y}) i+f_{2}(\mathbf{Y})(N-i)\right] N} P_{\mathbf{Y}}(\mathbf{y}) \mathrm{d} \mathbf{y} \\
& +g(1-i / N)
\end{aligned}
$$

where we use the abbreviation

$$
P_{\mathbf{Y}}(\mathbf{y}) \mathrm{d} \mathbf{y}=P_{Y_{1}}\left(y_{1}\right) P_{Y_{2}}\left(y_{2}\right) P_{Y_{3}}\left(y_{3}\right) P_{Y_{4}}\left(y_{4}\right) \mathrm{d} y_{1} \mathrm{~d} y_{2} \mathrm{~d} y_{3} \mathrm{~d} y_{4}
$$

and add $g(x)=\delta(x)$ to achieve reflecting boundaries ${ }^{3}$. The explicit form of the transition probabilities allows to calculate the anomalous

2 Periodic fluctuations with period $T=2$ can be reinterpreted as uncorrelated noise: The non-zero transition probabilities are $T(i \mid i+2), T(i \mid i)$ and $T(i \mid i-2)(T(i \mid i)$ does not appear in the simplified master equation). With $i^{\prime}=2 i$ we have the same situation as with random values from a probability distribution $P(x)=\delta(x+\sigma)+\delta(x-$ $\sigma)$.

3 For practical purposes (instead of a half delta function) we choose $g(x)=e^{-1000 x}$ which is differentiable and ensures reflecting boundaries. 


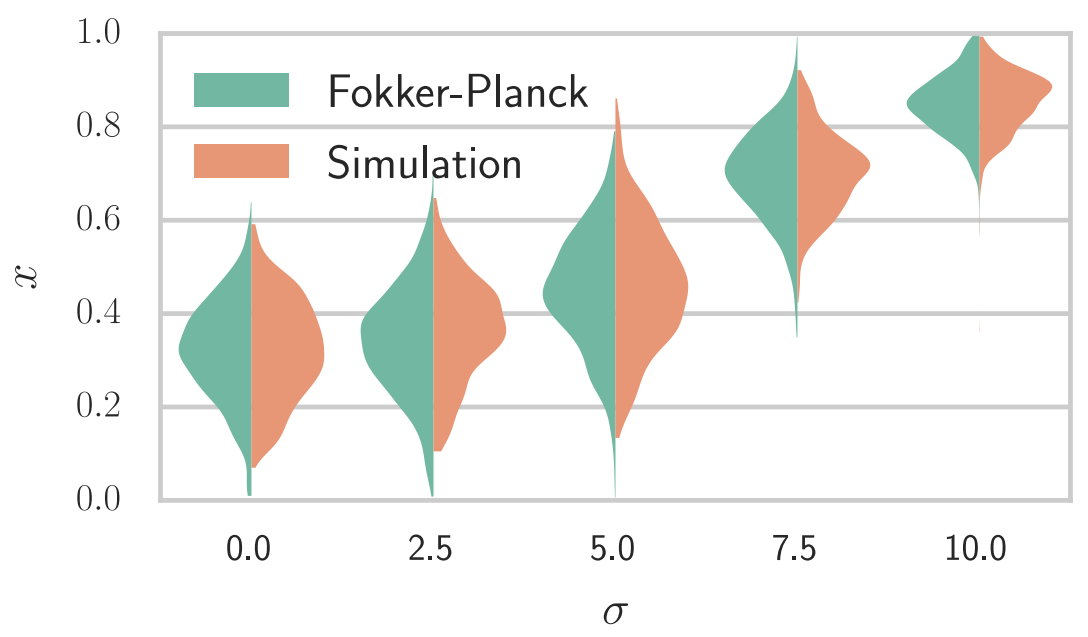

Figure 3.7: Anomalous stationary distributions of the Moran model: Theory and simulation. Stationary distributions calculated by the Fokker-Planck equation and measured stationary distributions using a simulation of the Moran model in comparison. The payoff matrix is $M^{(t)}=[6,5.5,7,5]+X^{t} \sigma[0,0,1,0]$, with $X^{t}$ randomly switching between +1 and -1 . The population size is $N=1000$.

stationary state as the solution of the Fokker-Planck equation for the Moran process [6]

$$
\partial_{t} \rho(x, t)=-\partial_{x}[a(x) \rho(x, t)]+\frac{1}{2} \partial_{x}^{2}\left[b^{2}(x) \rho(x, t)\right]
$$

which reads

$$
\rho^{*}(x)=\mathcal{N} \exp \left(\int_{0}^{x} \Gamma\left(x^{\prime}\right) \mathrm{d} x^{\prime}\right)
$$

for

$$
\mathcal{N}=\int_{0}^{1} \exp \left(\int_{0}^{x} \Gamma\left(x^{\prime}\right) \mathrm{d} x^{\prime}\right) \mathrm{d} x
$$

and

$$
\Gamma(x)=\frac{1}{b(x)}\left(2 a(x)-\frac{\mathrm{d} b}{\mathrm{~d} x}(x)\right),
$$

where $a(x)=T(x \mid x+1 / N)-T(x \mid x-1 / N)$, $b(x)=\sqrt{(T(x \mid x+1 / N)+T(x \mid x-1 / N)) / N}$, and $x=\frac{i}{N}$.

Figure 3.7 shows for an example how the stationary distributions, predicted by equation 3.29, change with increasing fluctuation intensities compared to stationary distributions of the simulated Moran model. 


\subsubsection{Correlated fluctuations}

Payoff values are not necessarily statistically independent from each other, as for example the payoff values $M_{11}$ and $M_{12}$ of the HawkDove game in figure 3.I. Thus, it is informative to study the effects of covariation. Consider the general case of a game specified by the payoff matrix $M=\left(\begin{array}{ll}Y_{1} & Y_{2} \\ Y_{3} & Y_{4}\end{array}\right)$. In order to show the impact of the correlations, we keep the intensity of the fluctuations equal and constant, $\sigma_{Y_{1}}=\sigma_{Y_{2}}=\sigma_{Y_{3}}=\sigma_{Y_{4}}=$ const. The correlations between $Y_{1}, Y_{2}$, $Y_{3}$ and $Y_{4}$ are specified by six independent correlation coefficients on which the resulting stationary states depend in a nonlinear way. For simplicity, figure 3.8 shows only the isolated impact of each pairwise correlation keeping the others zero.

This shows that in addition to intensities, the anomalous stationary states are crucially determined by the correlation of the fluctuations. Yet, we can show analytically that there is a special case $\left(\operatorname{corr}\left(Y_{1}, Y_{3}\right)=\operatorname{corr}\left(Y_{2}, Y_{4}\right)=1\right)$ for which the stationary state becomes completely independent of the fluctuation intensities. Assume that $\mathbf{x}^{*}$ is the stationary state of a game with constant payoff matrix $M_{0}$, such that $r\left(\mathbf{x}^{*}, M_{0}\right)=1$. If we add noise with correlation coefficient 1 between column values,

$$
M(t)=M_{0}+\tilde{M}(t)=\left(\begin{array}{ll}
a & b \\
c & d
\end{array}\right)+\left(\begin{array}{ll}
f_{1}(t) & f_{2}(t) \\
f_{1}(t) & f_{2}(t)
\end{array}\right),
$$

then

$$
\begin{array}{r}
r\left(\mathbf{x}^{*}, M_{0}+\tilde{M}(t)\right)=\frac{\left(M_{0} \mathbf{x}^{*}\right)_{i}+\left(\tilde{M}(t) \mathbf{x}^{*}\right)_{i}+b}{\mathbf{x}^{* T} M_{0} \mathbf{x}^{*}+\mathbf{x}^{* T} \tilde{M}(t) \mathbf{x}^{*}+b} \\
=\frac{\left(M_{0} \mathbf{x}^{*}\right)_{i}+\left(f_{1}(t) x_{1}^{*}+f_{2}(t) x_{2}^{*}\right)+b}{\mathbf{x}^{* T} M_{0} \mathbf{x}^{*}+\left(f_{1}(t) x_{1}^{*}+f_{2}(t) x_{2}^{*}\right)+b}=1
\end{array}
$$

where the second step uses $x_{1}^{2}+x_{1} x_{2}=x_{1}$ and $x_{2}^{2}+x_{1} x_{2}=x_{2}$ and the last step uses $\left(M_{0} \mathbf{x}^{*}\right)_{i}=\mathbf{x}^{* T} M_{0} \mathbf{x}^{*}$ following from the assumption. Consequently, in this case the stationary state does not depend on the noise intensity.

\subsubsection{Correspondence of payoff rank criteria and generalized criteria with constant payoffs}

In the case of constant payoffs, the payoff rank criteria and the generalized criteria of most games are equivalent. Only three pairs of games, Battle/Hero, Assurance/Coordination, and Harmony/Concord, have different payoff rank criteria but the same generalized criteria (see table 3.1). This reflects that the evolutionary dynamics of these games is structurally similar, as we explained for the Bat- 


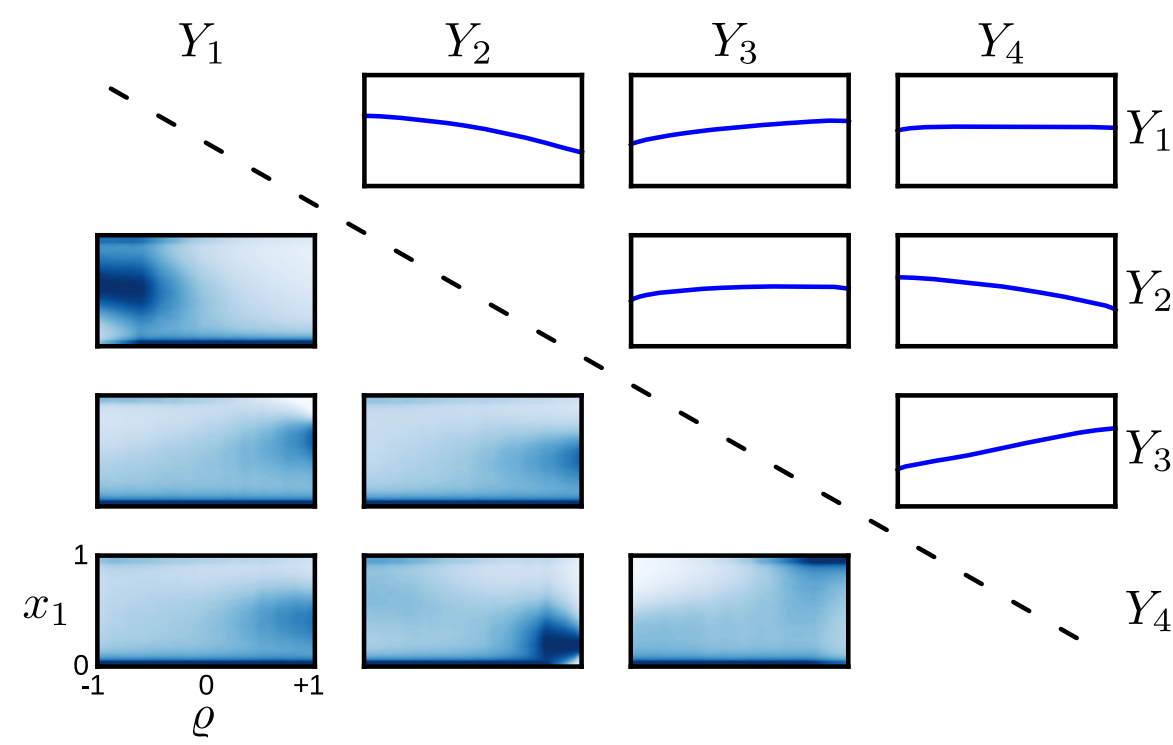

Figure 3.8: Impact of correlations. Periodic points $x^{\star}$ (upper part) and stationary distributions (lower part) for fluctuations with equal intensity $(\sigma=5)$ but different correlation coefficients $\varrho$. Each plot shows the impact of the correlation between two entries of the payoff matrix while keeping all other correlations zero.

tle/Hero pair in section 2.5.

Since the method to prove these relations is the same for all games we show it only for the Prisoner's Dilemma to exemplify the proof. We assume that the dynamics of the game are described by the continuous replicator equation $u_{1}:=\dot{x}_{1}=x_{1}\left((M \mathbf{x})_{1}-\mathbf{x}^{T} M \mathbf{x}\right)$ with a constant payoff matrix $M=\left(\begin{array}{ll}m_{1} & m_{2} \\ m_{3} & m_{4}\end{array}\right)$. According to the generalized criteria a Prisoner's Dilemma is defined as $[-2,+1,+1,+1]$. The -2 tells us that the first stationary state at $x_{1}=0$ is stable and the second at $x_{1}=1$ is unstable,

$$
\begin{gathered}
\frac{\mathrm{d} u_{1}}{\mathrm{~d} x_{1}}(0)<0 \Leftrightarrow m_{2}<m_{4} \\
\frac{\mathrm{d} u_{1}}{\mathrm{~d} x_{1}}(1)>0 \Leftrightarrow m_{3}>m_{1} .
\end{gathered}
$$

Further the three +1 tell us that the payoff of both players and the total payoff of the population at $x_{1}=1$ is higher than at $x_{1}=0$,

$$
\begin{aligned}
P_{1}(1)>P_{1}(0) & \Leftrightarrow m_{1}>m_{2} \\
P_{2}(1)>P_{2}(0) & \Leftrightarrow m_{3}>m_{4} \\
\langle P\rangle(1)>\langle P\rangle(0) & \Leftrightarrow m_{1}>m_{4} .
\end{aligned}
$$


Criteria (3.35) to (3.39) are equivalent to $m_{2}<m_{4}<m_{1}<m_{3}$ or in the payoff rank notation $[3,1,4,2]$, which defines a traditional Prisoner's Dilemma. 



\section{4}

\section{EVOLUTIONARY GAMES ON NETWORKS}

\section{I INTRODUCTION}

In chapter 3, we studied the effects of payoff fluctuations on evolutionary games in unstructured populations, meaning that every individual is assumed to interact with every other individual. Since many real organisms interact frequently only with a small subset of the whole population, for example because of social relations or geographical proximity, classical evolutionary game theory has been extended to evolutionary games on structured populations, meaning that individuals interact only with adjacent individuals on a network or with neighbors in a spatially distributed population [12-19, 73].

Even if the payoff values are constant, the payoff the individuals receive in an unstructured population are distributed, because the payoff depends on the number and the strategies of the individuals it interacts with, which can be different for every individual depending on the position in the structured population. This distribution of received payoffs can be considered as a payoff noise that is inherent to the population structure. Does this inherent payoff noise cause similar effects on the evolutionary dynamics as the payoff noise that we introduced in chapter 3 ?

In this chapter we argue that evolutionary games on networks differ from their mean-field solution in a similar way as an evolutionary game with payoff noise differs from an evolutionary game without payoff noise in unstructured populations.

\subsection{EXISTING RESULTS ON STRUCTURED POPULATIONS INDICATE EFFECTS OF INHERENT NOISE}

Evolutionary games on networks and spatially distributed populations show some remarkable similarities to our results for evolutionary games with payoff fluctuations.

In an evolutionary Prisoner's Dilemma with unstructured populations and without payoff fluctuations cooperation should die out. As we have seen in chapter 3, payoff fluctuations can change the dynamics from a Prisoner's Dilemma dynamics to some other game in which cooperation can persist. This observation is consistent with previous 
observations on the evolution of cooperation on networks or spatially distributed populations.

First, introducing migration in a spatially distributed population increases the inherent payoff noise, but strong migration, such that the interactions are completely random, leads effectively to the meanfield solution, where the inherent payoff noise is small. As this consideration suggests, it has been observed that small and intermediate migration enhances persistence of cooperation, while strong migration inhibits cooperation $[74,75]$.

Second, the inherent payoff noise should increase with the variance of the node degree in a network. Studies of evolutionary games in different network types show that in comparison to regular networks, in which the variance of the node degree is zero, scale-free networks, in which the variance of the node degree can be large or even diverging, promote cooperation [14, 76].

Third, in a fully connected network the inherent payoff noise is zero, which necessarily leads to the extinction of cooperators [14].

Fourth, in the public goods game, also known as the N-person Prisoner's Dilemma, social diversity in the sense of variable group sizes and variable number of games in which the individuals participate, promotes cooperation [77].

In summary, these observations indicate that the inherent noise in networks and spatially distributed populations have similar effects as payoff noise in unstructured populations.

\subsection{IMPLEMENTATION OF EVOLUTIONARY GAMES ON NETWORKS}

The content of this section is published in the ReScience Journal [78] (CC-BY 4.o) and reprinted here in a modified and rearranged form with additional information.

\subsubsection{Motivation}

In order to compare the effect of inherent payoff noise in networks with the effect of payoff fluctuations in unstructured populations, we implement the algorithms for the evolutionary process on networks that has been used in the seminal paper by Ohtsuki et al. on the evolution of cooperation in networks [16]. Although the idea of the algorithm is quite simple, the implementation is difficult, because accurate measurements require to run a large number of simulations on millions of different networks, which requires a very efficient and parallelized implementation. In this section, we describe the algorithm and use it to reproduce the numerical results of the paper by Ohtsuki et al. [16] to ensure that our implementation is correct. The source code of our implementation is available on GitHub ${ }^{1}$.

1 https://github.com/ReScience-Archives/Stollmeier-2017/tree/master/code 
4.3.2 Short review of Ohtsuki et al.: A simple rule for the evolution of cooperation on graphs and social networks (Nature, 2006)

A central question in evolutionary game theory is how cooperation can evolve in a Prisoner's Dilemma. In an unstructured population the defectors have always a higher payoff than the cooperators, hence natural selection leads to extinction of the cooperators. One of the mechanisms that promote cooperation is network reciprocity. If the individuals do not interact with all other individuals but only with a subset of the other individuals, e.g. the adjacent individuals in a social network, then cooperators can form groups with other cooperators, which increases the benefit from other cooperators and reduces the exploitation from defectors. As a consequence, the probability that cooperation evolves in networks can be larger than in unstructured populations. The probability, that an evolutionary process starting with a single cooperator in a population of defectors ends with a population of only cooperators is called the fixation probability (see figure 4.1). Cooperation is said to be favored by natural selection if the fixation probability exceeds $1 / N$, which is the fixation probability of a neutral mutation in a population of size $N$.

Beside the structure of the population, the fixation probability depends on the payoff values. In section 2.5 we introduced the Prisoner's Dilemma with four values in a $2 \times 2$-payoff matrix. Here, we use a less general parametrization of the Prisoner's Dilemma in order to reduce the number of variables,

$$
\begin{aligned}
& \text { Cooperate Defect } \\
& \text { Cooperate }\left(\begin{array}{cc}
b-c & -c \\
b & 0
\end{array}\right) \text { with } b>c>0 \text {, }
\end{aligned}
$$

where $b$ is the benefit that an individual receives from a cooperator and $c$ is the cost for cooperating with another individual.

Ohtsuki et al. [16] showed that a simple rule indicates whether cooperation is favored. For the death-birth update (explained below), the fixation probability of cooperators exceeds $1 / N$ if the condition $b / c>k$ is satisfied, where $k$ is the average node degree of the network. For imitation update (explained below), the fixation probability of cooperators exceeds $1 / N$ if the condition $b / c>k+2$ is satisfied.

A comparison of the fixation probabilities predicted by this simple rule and the fixation probabilities determined from numerical simulations shows that the rule is most accurate for regular networks with large populations. It is less accurate for networks in which the node degree varies, e.g. random or scale-free networks, and for networks with small population sizes. Until today, the question of the critical benefit-to-cost ratio $b / c$ in networks attracted much interest. Further proofs of this rule, more precise rules, exact calculations and general- 

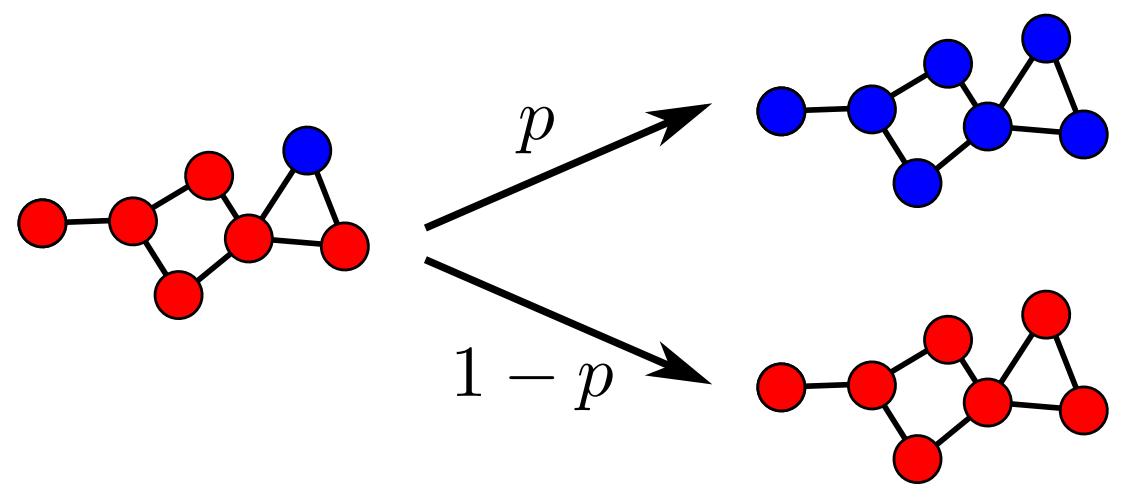

Figure 4.1: Illustration of the fixation probability. Starting the evolutionary process with only one cooperator at a random position leads with probability $p$ to a population of cooperators.

izations were published [19, 79-81]. Recently, Allen et al. derived an exact solution for the critical benefit-to-cost ratio under weak selection on arbitrary graphs [82].

\subsubsection{Description of algorithms}

\subsubsection{Measuring the fixation probability}

In order to measure the fixation probability for a certain graph type, we generate 1000 realizations of the graph type and start on each of these graphs 1000 evolutionary processes.

Each process starts with the initialization of the strategies. All individuals are set to "defect", except for one randomly chosen individual that is set to "cooperate". At each time step, the fitness of every node has to be calculated. According to the payoff matrix 4.1, a cooperator, which interacts with $m$ individuals of which $i$ are cooperators, receives the total payoff $P_{C}=b i-c m$, and a defector, which interacts with $m$ individuals, receives the total payoff $P_{D}=b m$. The fitness of an individual is $f_{X}=1-w+w P_{X}$, where $X \in\{C, D\}$ is its own strategy and $w$ is the selection strength and throughout this study set to 0.01 ("weak selection"). After the calculation of the fitness, the strategies of the nodes are updated (as explained below). The calculation of the fitness and the update is repeated until all individuals have the same strategy.

The fixation probability is the ratio of the number of processes in which all individuals cooperate at the end and the total number of the processes $(1000 \times 1000)$. For the simulations we use the same graph types as described in the supplementary of the paper by Ohtsuki et al. These graph types are circle graphs, lattice graphs, random regular graphs, random graphs and scale-free graphs, where each graph need to satisfy the condition that all nodes in the graph are part of 
one connected component to allow fixation at all [16].

\subsubsection{Death-birth update}

A death-birth update consists of two steps. First, a random individual is chosen to die. Second, the adjacent individuals compete for filling the vacant site with a copy of themselves. If the sum of the fitness of adjacent cooperators is $F_{C}$ and the sum of the fitness of adjacent defectors is $F_{D}$, the probability that the new individual is a cooperator is $\frac{F_{C}}{F_{C}+F_{D}}$.

\subsubsection{Imitation updating}

In imitation updating, the individuals do not die but imitate other strategies, which mean they can include their own fitness in their choice which strategy they want to imitate. First, a random individual is chosen to update its strategy. With the probability $\frac{F_{C}}{F_{C}+F_{D}+f_{0}}$ it will imitate the cooperation strategy and with the probability $\frac{F_{D}}{F_{C}+F_{D}+f_{0}}$ it will imitate the defection strategy.

4.3.4 Comparison of own implementation with results in the paper by Ohtsuki et al.

Before we can measure how the fixation probability depends on the benefit-to-cost ratio $b / c$, we need to choose absolute values for the $\operatorname{costs} c$ and the benefit $b$ for the simulations. Since only the ratio $b / c$ is given in the paper by Ohtsuki et al. [16], we first study how the choice of $c$ affects the results. As an example, we measure the fixation probability curves for the cycle graph with $N=500$ nodes and degree $k=10$ for different choices of $c$. The results are shown in figure 4.2 , together with data taken from figure $2 a$ of the paper by Ohtsuki et al. [16] for comparison.

Most importantly, the position of the intersection of the fixation probability curves with the fixation probability of a neutral mutation is almost unaffected by the choice of the absolute value $c$. Assuming that this is also true for other graph types, this means that we can choose (within a certain range) arbitrary absolute values. Since the curve with $c=0.125$ matches the data of the same graph type from Ohtsuki et al., we use this value for all further simulations.

Figure 4.3 shows the fixation probability curves for the death-birth process. As expected from the theory, all curves cross the line of a neutral mutation at benefit-to-cost ratios greater than $k$. With larger networks the intersection is much closer to $b / c=k$. This effect is much weaker for scale-free networks than for the other network types. These observations are consistent with the paper by Ohtsuki et al. [16]. The only visible difference is that the curves in the paper by Ohtsuki 


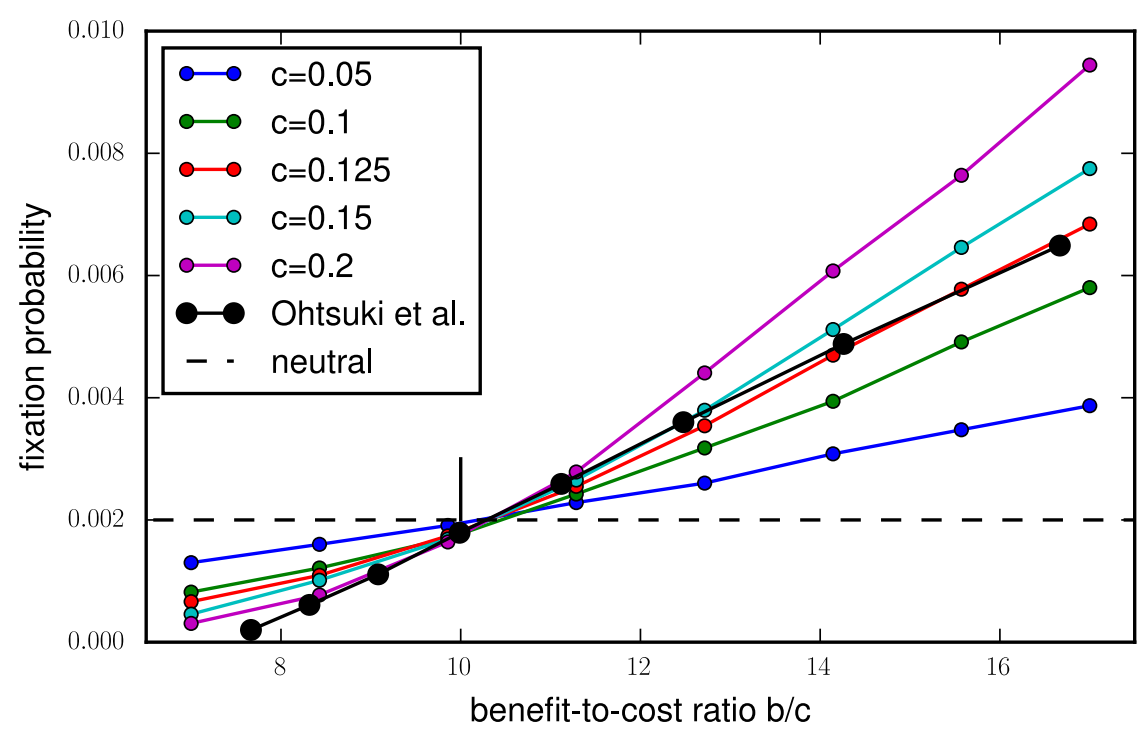

Figure 4.2: Fixation probability curves for a cycle graph with $N=500$ nodes and degree $k=10$. The colored dots are measured with different absolute cost values $c$, the black dots are taken from figure 2a of the paper by Ohtsuki et al. [16].

et al. are much steeper.

Figure 4.4 shows the fixation probability curves for imitation updating with small network sizes only, as in the paper by Ohtsuki et al. [16]. All curves cross the line of a neutral mutation at benefit-to-cost ratios greater than $k+2$. Again, the difference to the paper by Ohtsuki et al. is that the curves there are much steeper.

For all considered graph types, network sizes and degrees the positions of the intersection are consistent with the numerical results presented in the paper by Ohtsuki et al. and, with some deviations that depend on the graph type and the network size, are consistent with their theoretical results, $b / c>k$ for death-birth updating and $b / c>k+2$ for imitation updating. 

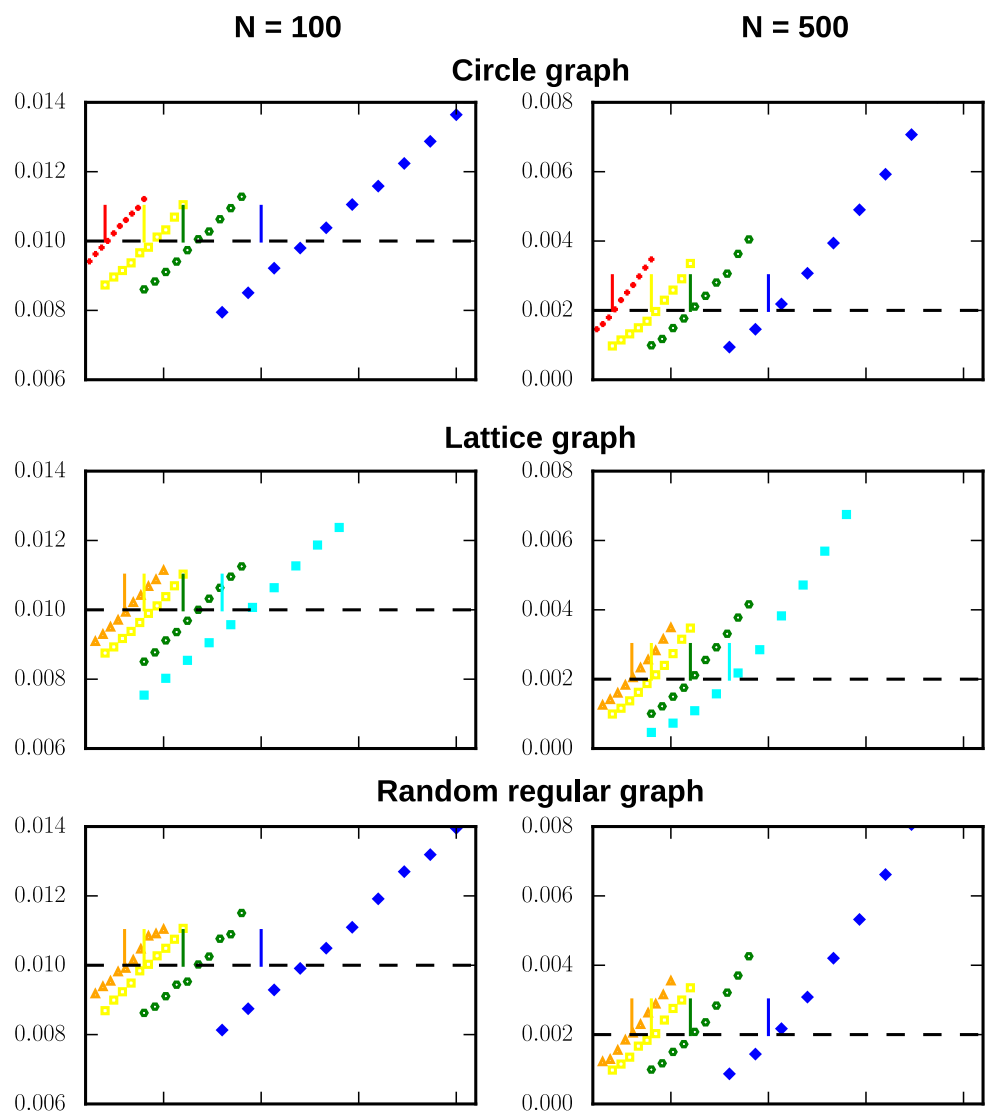

Random graph

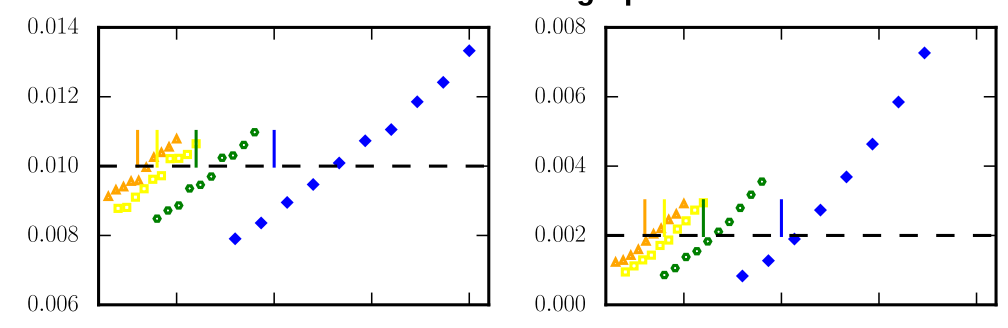

Scale-free graph
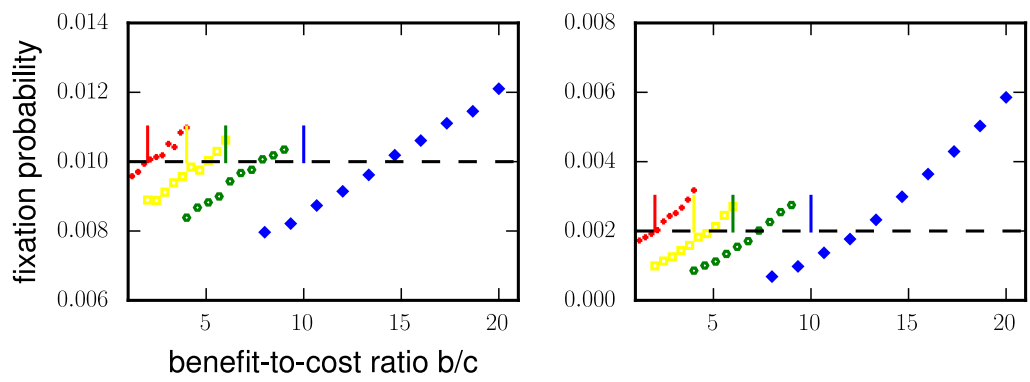

Figure 4.3: Fixation probability curves using death-birth updating with the same graph types and parameters as in figure 2 of the paper by Ohtsuki et al. [16]. The only parameter that is different to the paper by Ohtsuki et al. is the absolute cost value $c$, which is 0.125 here and unknown in the paper by Ohtsuki et al. The horizontal black dashed lines indicate the fixation probability of a neutral mutation $1 / N$, the vertical colored lines are at $b / c=k$. 

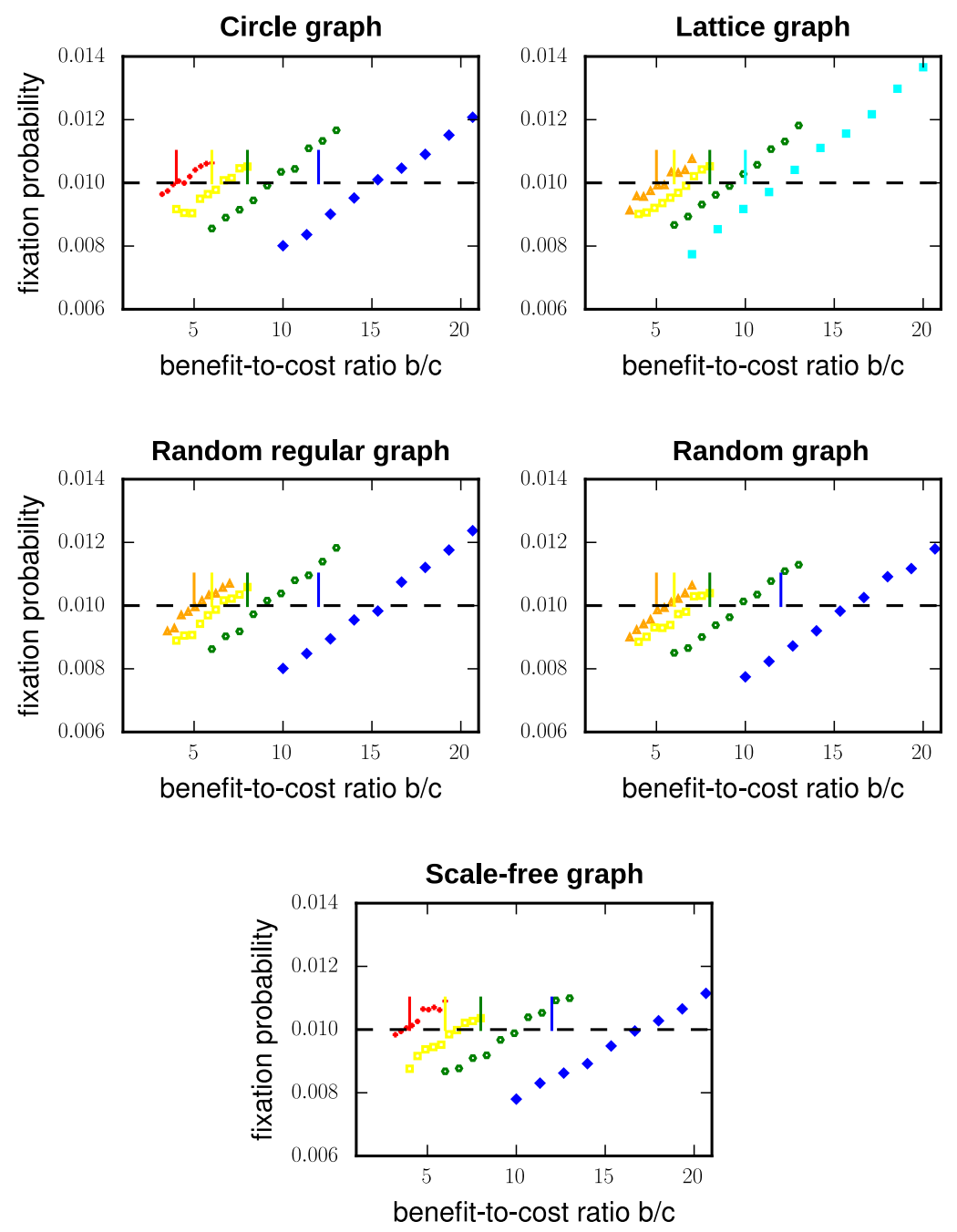

Figure 4.4: Fixation probability curves using imitation updating with the same graph types and parameters as in figure 4 of the supplementary of the paper by Ohtsuki et al. [16]. The only parameter that is different to the paper by Ohtsuki et al. is the absolute cost value $c$, which is 0.125 here and unknown in the original paper. The horizontal black dashed lines indicate the fixation probability of a neutral mutation $1 / N$, the vertical colored lines are at $b / c=k+2$. 


\subsection{THE EFFECT OF INHERENT PAYOFF NOISE ON EVOLUTION- ARY DYNAMICS}

\subsubsection{Methods}

To test the effects of inherent noise in evolutionary games on networks and compare the results with those from evolutionary games in unstructured populations with payoff fluctuations, we study the same four games that we used as examples in chapter 3 (figure 3.4). With the implementation described in section 4.3 (death-birth update variant), we simulate the evolutionary process of these four games on random networks.

During the evolutionary process, we measure the stationary probability distribution that a certain number of nodes is occupied by one strategy and we measure the transition probabilities, meaning the probabilities that the number of nodes occupied by one strategy increases or decreases by one. From these transition probabilities we can determine the states of detailed balance, which we can compare to the fixed points in unstructured populations.

Since the inherent noise is correlated with the average node degree, we vary the average node degree between a fully connected network (minimal inherent noise) and a network with a minimal node degree (maximal inherent noise). Note that the minimal node degree here is 2 , because all nodes of the network need to be connected to one component.

\subsubsection{Results}

Figure 4.5 shows how the variance of the inherent noise depends on the average node degree. As expected, the noise decreases with the node degree.

Figure 4.6 shows the measured stationary distributions and the states of detailed balance. For the Prisoner's Dilemma (A) we see that for large degrees (small noise) the network can typically accommodate only a small number of cooperators, while for small degrees (large noise) cooperation becomes substantially more abundant. This noise induced density dispersal is in qualitative agreement with the effects observed in chapter 3 for unstructured populations with variable payoffs (see figure 3.4A). For the Stag-Hunt game (B) and the Battle/Hero game $(\mathbf{C})$ the effect is rather weak so that the comparison to figure 3.4 is inconclusive. For the Hawk-Dove game (D) we see the stable state in the middle moving upwards where it meets with an unstable state, which looks quite similar to the first half of figure $3.4 \mathrm{D}$. 


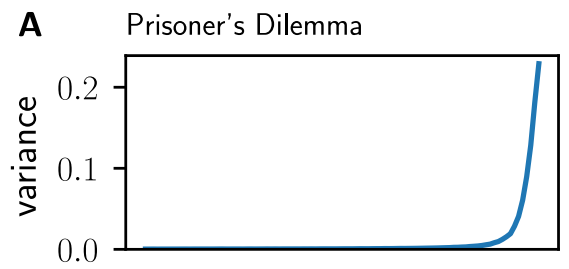

B Stag-Hunt

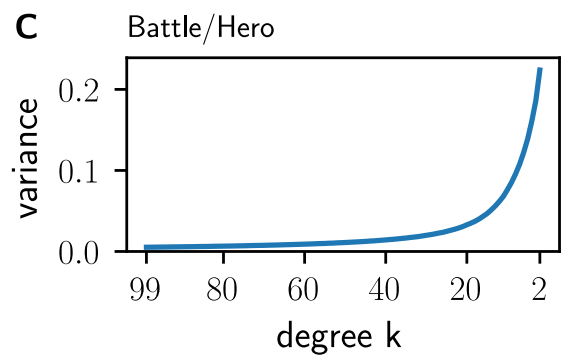

D Hawk-Dove

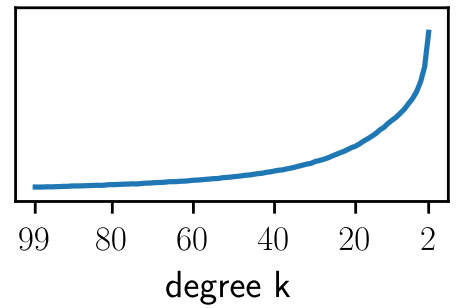

Figure 4.5: Variance of the cooperator's fitness in random networks with 100 nodes. The payoff matrices are $M=[3,1,4,2]$ in (A), $M=$ $[4,1,3,2]$ in (B), $M=[2,3,4,1]$ in (C) and $M=[3,2,4,1]$ in (D), which are the same as in figure 3.4 (with $\sigma=0$ ). Two curves are shown in (B), because the Stag-Hunt game has two stable fixed points, one where no individual cooperates and one where all individuals cooperate. The blue and the orange curves show the variance that was measured in the basin of attraction of the first and the second fixed point.

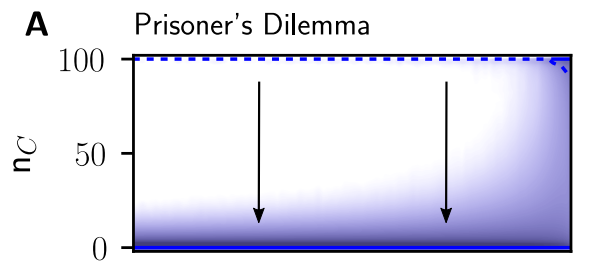

B Stag-Hunt
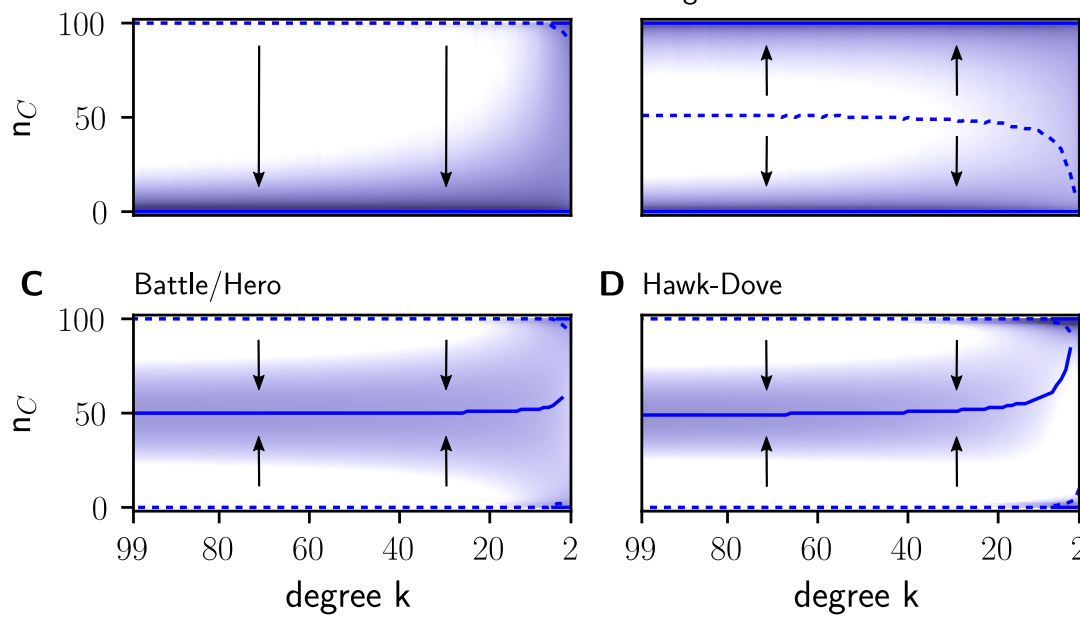

D Hawk-Dove

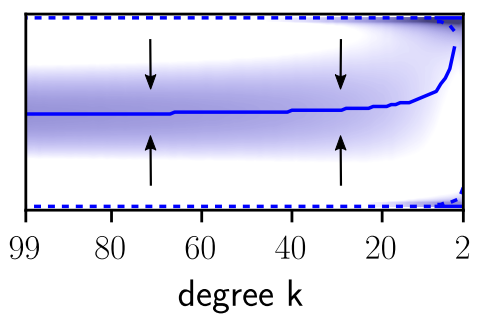

Figure 4.6: Stationary distributions and detailed balance in random networks with 100 nodes. Solid lines (stable equilibria) and dashed lines (unstable equilibria) represent detailed balance regarding the transition probabilities. Arrows indicate (in)stability. The payoff matrices are $M=[3,1,4,2]$ in (A), $M=[4,1,3,2]$ in (B), $M=[2,3,4,1]$ in (C) and $M=[3,2,4,1]$ in (D), which are the same as in figure 3.4 (with $\sigma=0$ ) and the same as in figure 4.6. 


\section{$4 \cdot 5$ DISCUSSION}

The similarities between the inherent noise in structured populations and the payoff noise in unstructured populations reported in section 4.2 and the comparison of our results in section 4.4 .2 with our results in chapter 3 indicate that the difference between an evolutionary game on structured populations and its mean-field solution is conceptionally the same as the difference between an evolutionary game in unstructured populations with a fluctuating payoff matrix and with a constant payoff matrix. This connects results from two fields that are usually studied separately. For instance, the evolution of cooperation in the Prisoner's Dilemma on networks, that is usually explained with network reciprocity, can also be explained with inherent payoff noise. This alternative interpretation offers a unifying perspective to understand the effects of network type, degree distribution and migration on the evolution of cooperation in structured populations.

However, we have demonstrated this analogy with only a few examples so far. A more systematic study is necessary to show how far the results generalize. In particular, it would be interesting to know if there are examples of networks and games where the results of the evolutionary game on the network and the evolutionary game in the unstructured population with fluctuating payoffs are exactly the same. If such examples exist, their analytical description, which is generally difficult on networks, could be translated to an evolutionary game in unstructured populations with payoff fluctuation, which is analytically manageable, as we have shown in chapter 3 . 



\subsection{MOTIVATION}

The results in chapter 3 show that environmental fluctuations can have a strong impact on the stationary states of evolutionary games. How can we observe these effects in a natural system and test the predictions of the theory?

Historically, evolutionary game theory has been developed largely without experimental evidence, because macrobiological species evolve so slow that most of them appear static to us. Instead, evolutionary game theory was often used as an explanation how the static observations may have evolved. For example, the fights between male deers and the competitions between birds for the best nest locations have been explained as Hawk-Dove games [50, 51], the mating behavior of lizards has been explained as a rock-paper-scissor game [83], and the competitions between plants for space for their roots and for light for their leafs have been explained as tragedies of the commons [84, 85]. Compared to these macrobiological systems, it is much easier to study the evolution of microbiological systems in experiments, because their reproduction cycles are short enough to get hundreds of generations within a few days and it is much easier to repeat experiments in controlled conditions. A number of experiments demonstrated that viruses [53], yeast cells [52], and bacteria [86-89] can be involved in evolutionary games that are similar to classical evolutionary games like the Prisoner's Dilemma or the Hawk-Dove game.

Theoretically, each of these systems could be affected by fluctuating environmental conditions. However, in order to study the impact of fluctuating environmental conditions, we need phenomenological models that describe the evolutionary games. A Hawk-Dove game may serve as an intuitive explanation for interactions between bacteria or yeast cells, but it is not a realistic model.

In this chapter we aim to develop a realistic phenomenological model of the evolution of shared antibiotic resistance in bacteria in order to bridge the gap between evolutionary game theory and real evolutionary games.
The content of this section is based on an article that is submitted to Physical Review X and presented here in a slightly modified and rearranged form with some additional information. 


\subsection{INTRODUCTION}

Antibiotic resistance can be a social behavior. Experiments have demonstrated that one bacteria strain can produce enzymes to inactivate the antibiotic and this way "cooperate" by protecting the other strain [8891]. Even if the other strain is not resistant, in coexistence it is able to sustain the antibiotic-polluted environment. The coexistence of the two strains can be an evolutionarily stable state. Bacteria that cannot produce the enzymes suffer from the antibiotic if the density of producers is insufficient but they have an advantage otherwise because they do not lose energy in producing the enzyme. Thus, sensitive strains may survive a large dose of antibiotics, and their populations quickly recover after the treatment. On the other hand, shared antibiotic resistance could help to maintain an acquired resistance during a phase of relatively low antibiotic concentrations because as long as the sensitive bacteria need the protection from resistant bacteria they cannot completely outcompete them.

Experiments showed that coexistence in isolated and controlled conditions can be an evolutionarily stable state [88-91]. Further, a recent study of the pairwise interactions between separated pure strains (bacterial isolates) from patients with polymicrobial infections showed that cooperative antibiotic resistance may be common in the wild [92]. On the other hand, strains that inactivate antibiotics cannot necessarily coexist with strains that do not inactivate antibiotics [90, 91].

The competitive exclusion principle states that two species competing for the same resource should not coexist. Many mechanisms are known that nevertheless maintain species diversity [93], including temporal niches [94], spatial dynamics [95] and rock-paper-scissor dynamics $[86,96]$. In addition, it has been shown that coexistence of three or more species in a well-mixed environment is possible with three-way interactions, such as in a community of antibioticproducing, antibiotic-degrading and antibiotic-sensitive bacteria [97]. However, the coexistence of antibiotic-degrading and antibiotic sensitive bacteria strains as observed in the experiments mentioned above involves only two phenotypes in a well-mixed environment. The mechanism that can explain this type of coexistence consists of an external influx of antibiotic which inhibits one strain and is degraded by the other strain $[98,99]$. But it remains unknown which phenotypes allow two bacteria strains to coexist, whether a third phenotype can invade a community of two coexisting strains, and whether coexisting strains can be mutationally stable.

Traditionally, resistance is described as a single-valued attribute, e.g. the minimum inhibitory concentration (MIC), which is the lowest concentration of the antibiotic which prevents growth. In this chapter, we argue that we need to describe antibiotic resistance as a two dimensional phenospace to understand shared antibiotic resistance. 
Based on this hypothesis, we present a model for shared antibiotic resistance that reproduces a number of well known phenomena of bacterial antibiotic-resistance and predicts which phenotypes can coexist and which phenotypes can not coexist. We further show that, although shared antibiotic resistance can be an evolutionarily stable state, it is not mutationally stable. Surprisingly, even an antibioticsensitive bacteria strain can invade and replace a pair of coexisting strains.

\subsection{THE PHENOSPACE OF ANTIBIOTIC RESISTANCE}

Self-limited antibiotic resistance results from a combination of mechanisms that protect the bacteria from the antibiotic. In an antibioticpolluted environment, bacteria with a tolerance level above the antibiotic concentration can reproduce but those bacteria with a tolerance level below can not reproduce. This results in a selective pressure towards the evolution to higher resistance that increases with the antibiotic concentration. In contrast, for bacteria with high tolerance in a low antibiotic concentration, there is a selective pressure towards the loss of resistance since resistant bacteria tend to have a smaller fitness (through a higher cost) than less resistant bacteria. Yet, most studies find this selective pressure inferior to the other direction (evolving resistance is usually faster than loosing resistance) [10o]. Invariably, if the antibiotic concentration is constant over a long time, the smallest sufficient level of resistance is expected to be mutationally stable.

Shared antibiotic resistance and also the observation that resistance may increase with population size (known as the "inoculum effect"), do not fit into this picture of self-limited antibiotic resistance. The tolerance level in shared antibiotic resistance depends not only on the bacteria itself and is therefore not a useful characteristic to describe the resistance phenotype of a bacteria. To attack this problem we propose a model where the large diversity of mechanisms to increase resistance are described by two phenomenologically distinct types. The first one reduces the burden of the antibiotic. Mechanisms of this type include a lower cell wall permeability to hinder the antibiotic to enter the cell, efflux pumps to remove the antibiotic from the cell and target modification or changing the metabolic pathway to reduce the vulnerability to the antibiotic.

The second type of mechanisms inactivates the antibiotic. Examples are bacteria that produce $\beta$-lactamase enzymes to degrade $\beta$-lactam antibiotics [88-90], bacteria expressing chloramphenicol acetyltransferase to inactivate the bacteriostatic antibiotic chloramphenicol [91], or simply an overproduction of the antibiotic binding target proteins. This, in fact, suggests to quantify the resistance phenotype as a combination of avoiding and inactivating the antibiotic. 


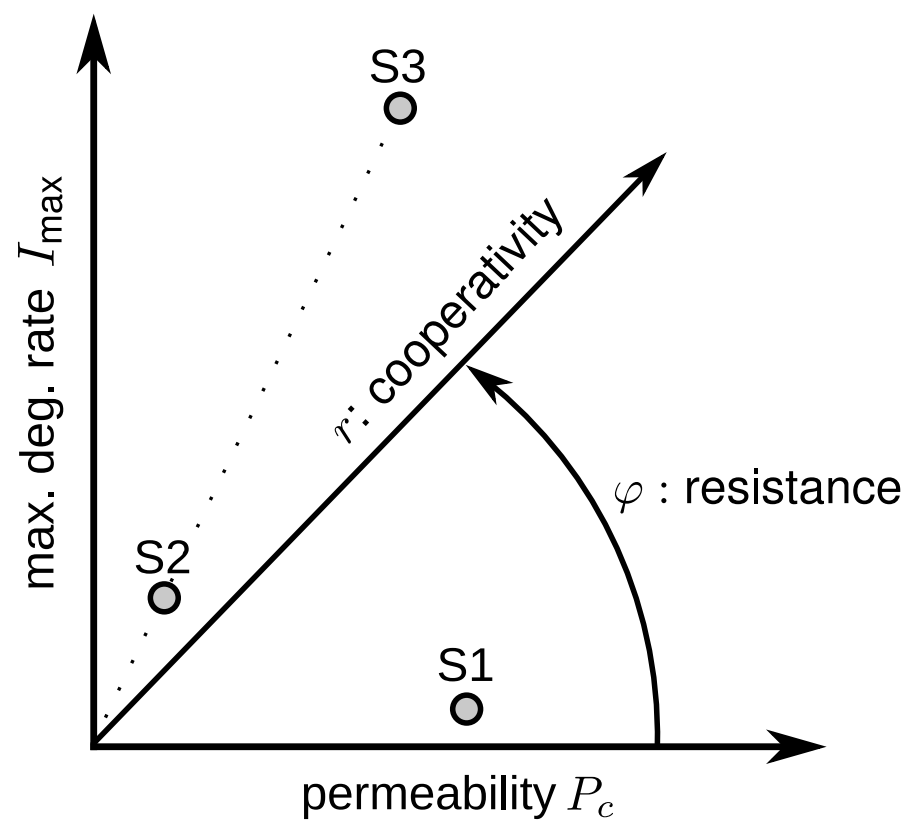

Figure 5.1: Resistance phenospace. The Cartesian coordinates are the physical properties of the bacteria, the permeability of the cell wall for the antibiotic and the maximum antibiotic degradation rate. The polar coordinates are the "behavioral" properties, the maximal tolerable extracellular antibiotic concentration ("resistance") and the degradation rate depending on the extracellular concentration ("cooperativity"). Three prototypical phenotypes illustrate the characteristics of the phenospace: $\mathrm{S}_{1}$ is a very sensitive strain because of the high permeability and the low maximum degradation rate. $S_{2}$ is resistant and non-cooperative because of the low permeability and the low maximum degradation rate (a combination that is called "self-limited antibiotic resistance"). $\mathrm{S}_{3}$ is resistant and cooperative because of the high permeability and the high maximum degradation rate (a combination that is called "shared antibiotic resistance"). S2 and S3 have exactly the same resistance, but the $S_{3}$ strain is much more cooperative than the S2 strain.

\section{$5 \cdot 4$ CELL MODEL}

As the two properties that describe the ability to avoid and inactivate the antibiotic we choose the cell wall permeability, denoted as $P_{c}$, and the maximal ability to degrade antibiotics, denoted as $I_{\max }$. If the bacterium is exposed to an extracellular antibiotic concentration $c_{\mathrm{ec}}$, the antibiotic diffuses through the cell wall into the cell where it is either degraded or accumulates - resulting in cell survival or cell death, respectively. To prevent the accumulation of the antibiotic in the cell it has to degrade the antibiotic with the same rate as it diffuses through the cell wall, $I=-P_{c}\left(c_{\mathrm{ic}}-c_{\mathrm{ec}}\right)$. We can assume that the cell dies if the intracellular concentration $c_{\mathrm{ic}}$ exceeds zero. Then the maximal extracellular concentration that the cell can cope with is $\max c_{\mathrm{ec}}=\frac{I_{\max }}{P_{c}}$. 

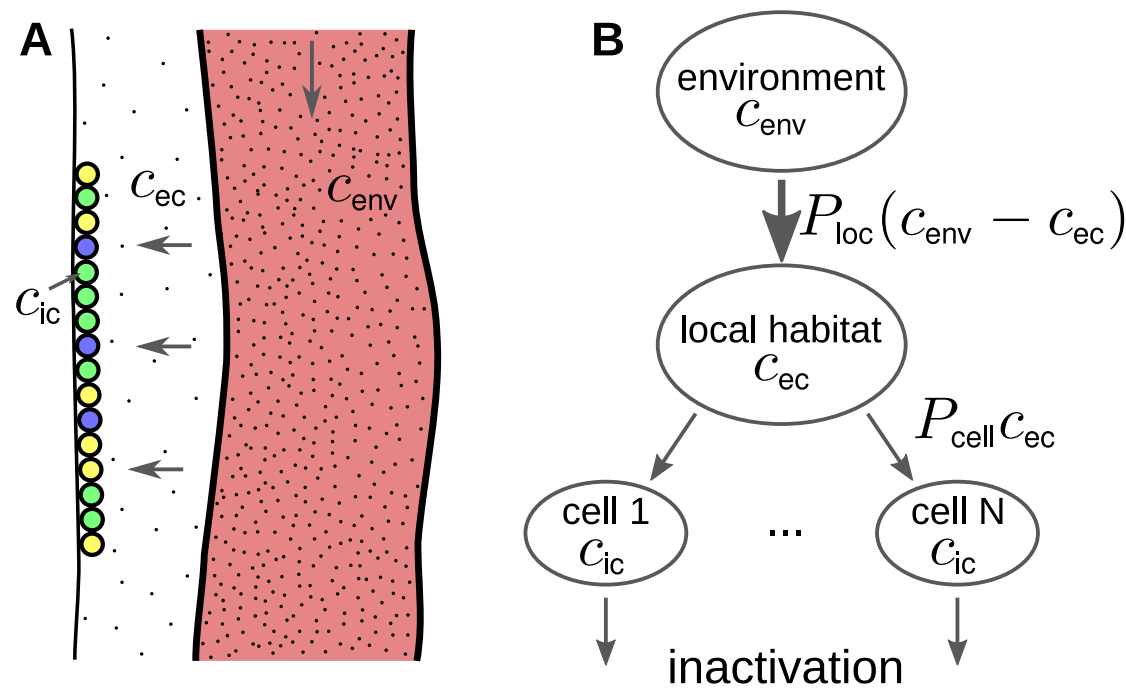

Figure 5.2: Illustration and specification of the colony model. (A) Illustration of an extravascular bacteria colony of different phenotypes (colored circles). An antibiotic (black dots) diffuses first from the blood to the extracellular space and then to the intracellular space of the bacteria, where it is degraded. (B) A corresponding multi-compartment model. The antibiotic from the environment (e. g. the blood vessel) diffuses through a barrier with permeability $P_{\text {loc }}$ to the local habitat of the bacteria and then through the cell walls with permeability $P_{\text {cell }}$ inside the bacteria cell, where it is degraded.

Compare two strains, one having the maximal degradation rate $I_{\max }$ and the permeability $P_{C}$, and the other strain having $2 I_{\max }$ and $2 P_{C}$. Since the maximal tolerable concentration is the ratio $I_{\max } / P_{C}$, both strains have the same resistance. The difference between the two is that the first will degrade the antibiotic slower than the second strain. Hence the latter is more cooperative. Thus, our model bacteria can be characterized either by Cartesian coordinates, with permeability and maximum degradation rate as the two axes, or by polar coordinates, with cooperativity as the radial coordinate and resistance as the angle (i.e. $\tan \varphi=I_{\max } / P_{C}$, with $0 \leq \varphi \leq \pi / 2$ ) as shown in figure 5.1.

\section{$5 \cdot 5$ COLONY MODEL}

For shared antibiotic resistance we assume that the degradation by one bacterium lowers the extracellular concentration of neighboring bacteria, due to a flow barrier with a certain permeability or just a larger distance between the colony and the antibiotic source compared to the mean distance between the bacteria. In general, the colony lives in a small habitat with a local concentration that is diffusively coupled to its environment. We assume that the local concentration in the habitat is homogeneous (all bacteria have the same 


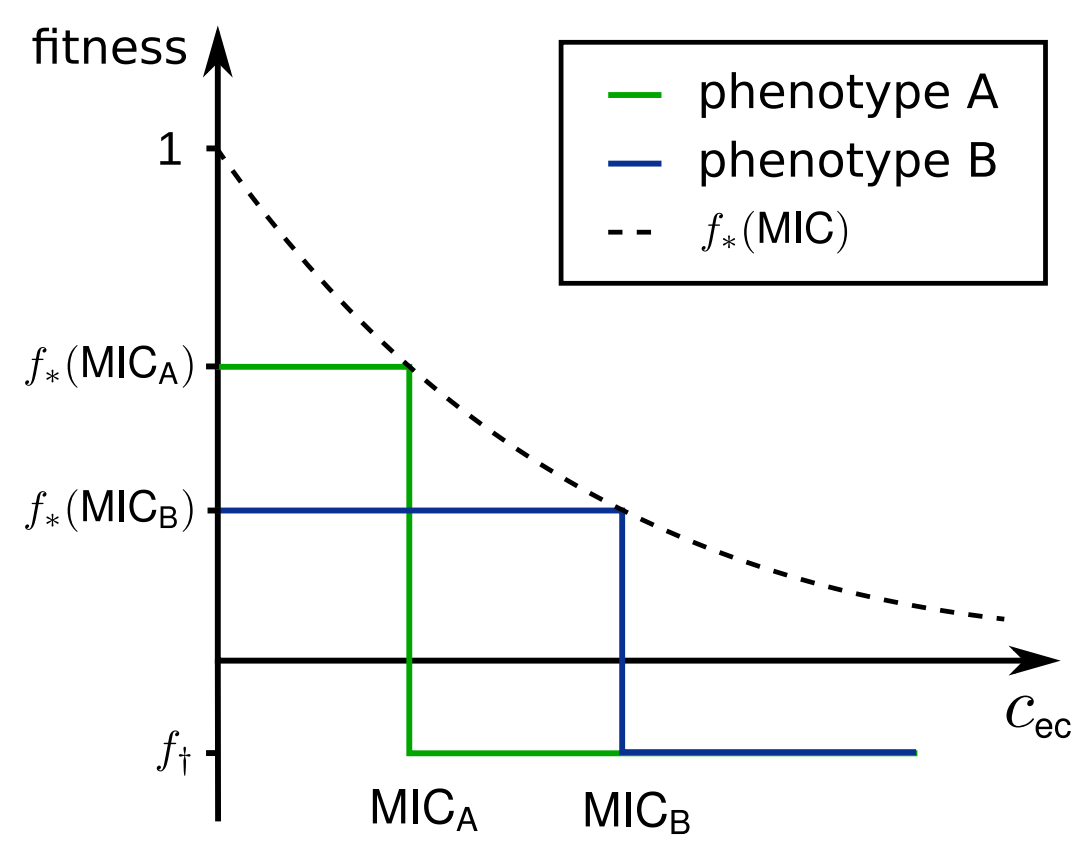

Figure 5.3: Fitness landscape. The green and blue line show the fitness function of two different phenotypes. Both phenotypes have a positive fitness $f_{*}$ if $c_{\mathrm{ec}}<$ MIC and a negative fitness $f_{+}$if $c_{\mathrm{ec}}>$ MIC. The negative fitness $f_{+}$is always the same, but the positive fitness $f_{*}$ is a function of the MIC (dashed black line).

extracellular concentration) and that the environment is an infinitely large reservoir with a constant concentration.

Since the bacteria degrade the antibiotic there will be a steady flux of antibiotic from the environment to the local habitat into the cells, as shown in figure 5.2. The total degradation rate is $I_{\mathrm{deg}}=N \sum_{i=0}^{m} n_{i} I_{i}$, where $N$ is the total number of cells, $n_{i}=\frac{N_{i}}{N}$ is the population share of phenotype $i, I_{i}=P_{c, i} c_{\mathrm{ec}}$ is the inactivation rate of a single cell of phenotype $i$ and the sum goes over all $m$ present phenotypes. The influx of antibiotic from the environment $I_{\text {in }}=P_{\text {loc }}\left(c_{\text {env }}-c_{\text {ec }}\right)$ depends on the permeability $P_{\mathrm{loc}}$ and the concentration gradient between habitat and environment. If the concentration in the environment does not change, then the concentration in the local habitat approaches a stationary state, where $I_{\mathrm{deg}}=I_{\mathrm{in}}$. This balance gives the stationary concentration

$$
c_{\mathrm{ec}}^{*}=\frac{P_{\mathrm{loc}} \mathcal{C}_{\mathrm{env}}}{P_{\mathrm{loc}}+N \sum_{i=0} n_{i} P_{c, i}} .
$$




\subsection{FITNESS LANDSCAPE}

In order to study the evolutionary dynamics it is essential to define a fitness function that depends on the antibiotic concentration and the phenotype alone.

The definition of the minimal inhibitory concentration (MIC) implies that the fitness is positive if the antibiotic concentration is below the MIC and negative if the antibiotic concentration is above the MIC. The transition close to the MIC is a rather sudden decline [101, 102], which we model as a discontinuous step exactly at the MIC.

It is known that bacteria strains with a high level of resistance tend to have a smaller fitness than bacteria strains with a low level [100]. Hence we assume that the positive fitness for antibiotic concentrations below the MIC is a function that is monotonously decreasing with the MIC.

For an illustration of the fitness functions see figure 5.3.

If the environmental conditions are constant, details of the actual functional form and the absolute values of the fitness are not relevant for the stationary states of the evolutionary dynamics. Relevant is whether the fitness is positive or negative, whether it is greater or smaller compared to the fitness of another strain, and at which antibiotic concentration their fitness functions intersect.

\section{$5 \cdot 7$ EVOLUTIONARY DYNAMICS}

\subsubsection{Strain A alone}

First, let us assume that initially $N$ bacteria of only one phenotype $A$, specified by the tuple $\left(I_{\max , A} ; P_{c, A}\right)$, live in an environmental antibiotic concentration $c_{\mathrm{env}}$. The population will survive if $c_{\mathrm{ec}} \leq \mathrm{MIC}=\frac{I_{\mathrm{max}, \mathrm{A}}}{P_{c, A}}$. Using equation 5.1 and solving for $I_{\max , \mathrm{A}}$ determines the survival condition

$$
I_{\max , A}\left(P_{c, A}\right) \geq \frac{P_{\mathrm{loc}} P_{c, A} c_{\mathrm{env}}}{N P_{c, A}+P_{\mathrm{loc}}}
$$

Figure 5.4A shows how this condition separates the phenospace in a region of phenotypes that can survive in this habitat (red area) and phenotypes that will not survive (white area).

\subsubsection{Strain $A$ invaded by $B$}

Here we assume that the strain with phenotype A is able to survive and ask whether a second strain with a different phenotype $B$, $\left(I_{\max , B} ; P_{c, B}\right)$, can invade this population and if it does, whether it 
A

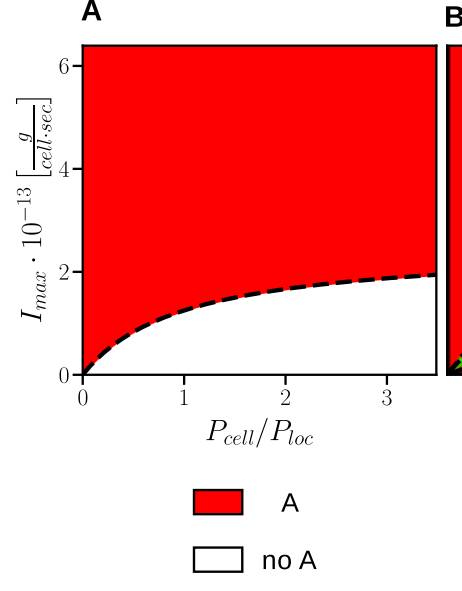

B

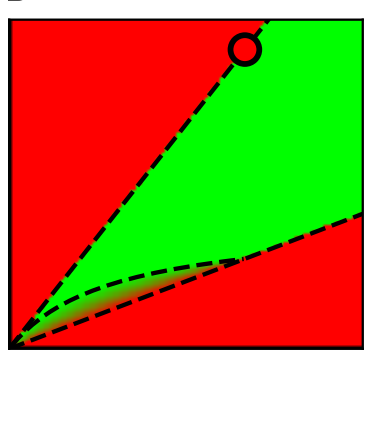

$B^{\prime}$

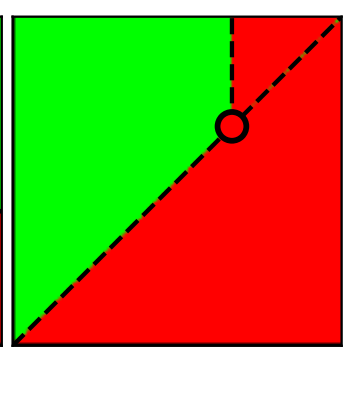

C

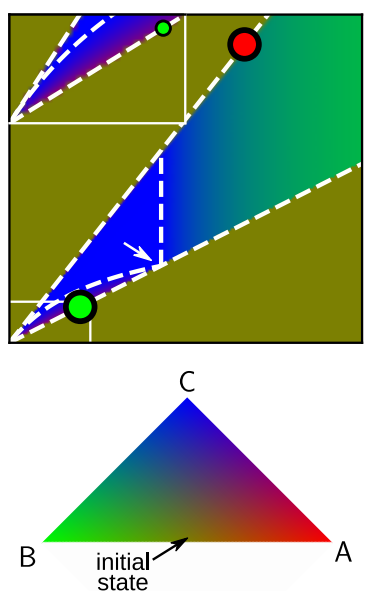

Figure 5.4: Stationary states after invasion. The axes are the relative cell permeability and the maximal degradation rate, as explained in Fig. 5.1. For the exact parameters of the environment and the phenotypes used here see appendix A.1. Dashed lines are the boundaries between the areas obtained from theory (section 5.7). (A) shows the stationary state after the invasion of phenotype A in an uninhabited antibiotic-polluted environment. The red area represents the phenotypes that can colonize the environment and the white area contains the phenotypes that die. In (B) we assume that the environment is already colonized by a phenotype $\mathrm{A}$ (depicted by the red filled circle) with $\mathrm{MIC}_{\mathrm{A}}>c_{\mathrm{ec}}$. Phenotypes in the red area are not able to invade the population of A. Phenotypes in the green area completely replace A. Phenotypes in the small area of gradual transition from red to green do invade A and form a stable coexistence with A. In the third plot $\left(\mathbf{B}^{\prime}\right)$ we assume that the environment is already colonized by a phenotype $\mathrm{A}$ (depicted by the red filled circle) with $\mathrm{MIC}_{\mathrm{A}}=c_{\mathrm{ec}}$. Again, phenotypes in the red area can invade and phenotypes in the green area cannot invade the population of A. In (C) we assume that two phenotypes, A and B, coexist with each other. Phenotype A, depicted by the circle filled with red, is the same as in plot (B). Phenotype B, depicted by the green filled circle is in the middle of the area of coexistence in plot (B). Phenotypes in the olivegreen area are not able to invade. At the bottom left is a small area with a gradual transition from red to blue (the inset shows a part of this region enlarged), meaning that the phenotype $C$ will replace $B$ and coexist with $A$. The area above the curved dashed line is completely blue, meaning that phenotype $\mathrm{C}$ replaces $\mathrm{A}$ and $\mathrm{B}$. The gradient from blue to green on the right hand side of the vertical dashed line shows that here phenotype $C$ will coexist with phenotype $B$ in varying ratios decreasing from left to right. As an example, the invasion process at the position marked by the white arrow is shown in detail in Fig. 5.5. 
outcompetes A or coexists with A. Species that have a higher fitness reproduce faster, as formalized by the replicator equation

$$
\frac{\mathrm{d} n_{i}}{\mathrm{~d} t}=n_{i}\left(f_{i}-\langle f\rangle\right),
$$

where $\langle f\rangle=\sum n_{i} f_{i}$ is the average fitness of the population. A strain of type $B$ can invade the population of $A$ if $\frac{\mathrm{d} n_{B}}{\mathrm{~d} t}>0$ for $n_{B}=0$ (and $\left.n_{A}=1-n_{B}=0\right)$. If $\frac{\mathrm{d} n_{B}}{\mathrm{~d} t}=0$ for any $n_{B} \in(0,1)$ the successful invasion leads to a stable coexistence of the two strains and if $\frac{\mathrm{d} n_{B}}{\mathrm{~d} t}>0$ for all $n_{B} \in(0,1)$ a strain of type B will replace any strain of type A.

Using this formalism we can divide the phenospace in regions as depicted in figure $5.4 \mathrm{~B}$. The result is intuitive. A strain of type B in the upper red region (above a line from the origin through the position of A) has a higher MIC than A and therefor a lower fitness, hence it cannot invade a population of A. Strains of type B in the lower red region have an MIC that is smaller than the antibiotic concentration. Between these red regions there is a large green region where a strain $B$ has an MIC larger than the antibiotic concentration but smaller than the MIC of A, hence B has a higher fitness and outcompetes A. Strains between the dashed and the solid line can invade and form a stable coexistence with A. Strains of type B in this region are less active in the degradation than those of type A. As a consequence, the extracellular concentration will increase with the increasing population of $\mathrm{B}$ (and decreasing population of A). At some point, the extracellular concentration exceeds the MIC of $\mathrm{B}$, which leads to an decrease of $\mathrm{B}$ (and increase of $\mathrm{A}$ ), and a decreasing extracellular concentration. Hence the population ratio of $\mathrm{A}$ and $\mathrm{B}$ has a stable coexistence at that point where extracellular concentration equals the MIC of B.

\subsubsection{Strain $A$ and $B$, invaded by $C$}

Next, we assume that we have two coexisting strains A and B and ask whether a third strain $C$ can invade. If it does, can it coexist with either or both of the residents? Strain $C$ can invade, if $\frac{\mathrm{d} n_{C}}{\mathrm{~d} t}>0$ for $n_{C}=0$ and $n_{A}+n_{B}=1$. After a successful invasion, the system goes from the initial state to the first stable stationary state, where $\frac{\mathrm{d} n_{A}}{\mathrm{~d} t}=\frac{\mathrm{d} n_{B}}{\mathrm{~d} t}=\frac{\mathrm{d} n_{C}}{\mathrm{~d} t}=0$. Figure $5.4 \mathrm{C}$ shows these stationary states. The color at the position of a potential phenotype $C$ takes the RGB value from the composition of the three populations $\left(n_{A}, n_{B}, n_{C}\right)$ in these stationary states. The general picture can be understood similarly to the previous case of two strains. A strain $C$ in the upper region (above the line from the origin through the phenotype of $\mathrm{A}$ ) has a larger MIC than A, therefore has a smaller fitness and is unable to invade. A strain of type $C$ in the lower region (below a line from the origin through the phenotype B) has an MIC that is smaller than the extracellular concentration. Only between these two regions 


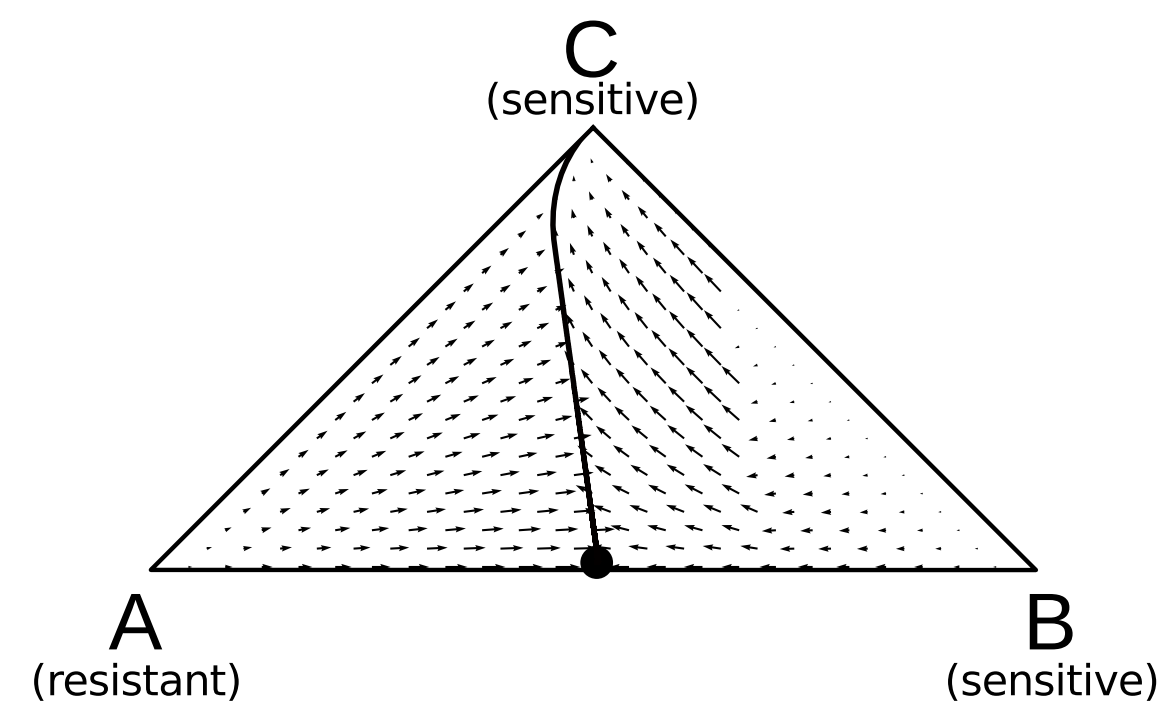

Figure 5.5: Example trajectory through a phase space of three phenotypes. Visualization of the invasion process happening in figure $5.4 \mathrm{C}$ at the position marked by the white arrow. Strain A and B can coexist and survive in high antibiotic concentrations $\left(\mathrm{MIC}_{\mathrm{A}}=5\right.$ and $\mathrm{MIC}_{\mathrm{B}}=2$ ). At the state of stable coexistence of $\mathrm{A}$ and $\mathrm{B}$, a single cell of strain $C$ appears in the population. This state is the initial state marked by the black circle. The black line shows how the population shares change. Although strain $C$ is not resistant $\left(\mathrm{MIC}_{\mathrm{C}}=2.3\right)$, it successfully invades the population and eventually completely replaces A and B.

$C$ can invade, but here its fate depends on how the invasion changes the populations of $\mathrm{A}$ and $\mathrm{B}$ and how this affects the extracellular concentration. Note that we observe points where only A, B or C survives and points where two of them coexist, but there is no phenotype $\mathrm{C}$ whose invasion leads to a coexistence of all three strains. Also note that in the blue area, where only strain $C$ survives the invasion, $C$ has just a slightly larger MIC than B. In other words, a strongly and a weakly resistant strain that live in a stable coexistence can be completely replaced by a strain of weak resistance.

\subsection{MUtational Stability AND the DiRection OF EVolu- TION}

So far we considered the dynamics of arbitrary but immutable phenotypes. The fixed points of this system are evolutionarily stable states, meaning that the ratio of the subpopulations is restored after a small perturbation. However, these states are not necessarily mutationally stable, where the phenotype would be restored after a small perturbation caused by mutations. For simplicity we assume that mutations are so infrequent that between two mutations the system approaches the evolutionarily stable state. 
In the case of one phenotype $\mathrm{A}$, we read off from figure $5.4 \mathrm{~B}$ that mutants of A with a slightly smaller resistance can replace A but mutants with a slightly larger resistance cannot replace A. After mutants with a smaller resistance have successfully replaced A the situation is essentially restored, except for the decreased level of resistance and a different cooperativity resulting from a random walk. The cooperativity changes randomly due to our assumption that the fitness is independent of the cooperativity. Since a change of cooperativity results in a change of the total degradation rate, the extracellular concentration of antibiotic is also affected. This process towards lower resistance continues until the difference between the MIC and the extracellular concentration of the antibiotic has vanished. Note that the mutants with slightly smaller resistance have only a slightly larger fitness. Therefore the reduction of resistance is a very slow process. When the evolved level of resistance reached the extracellular concentration, the mutations with larger cooperativity cannot replace A (figure $\left.5.4 \mathrm{~B}^{\prime}\right)$, hence the mutations move the population towards smaller cooperativity. This causes a small but successive increase of the extracellular concentration and therefore also a small increase of resistance. The final mutationally stable state is reached when the cooperativity is zero.

In the case of two coexisting phenotypes, we have to examine both for mutational stability. Consider the situation in figure $5.4 \mathrm{C}$, where $\mathrm{A}$ is the resistant and cooperative phenotype and $\mathrm{B}$ is the sensitive and non-cooperative phenotype.

First, assume that the phenotype of $B$ is fixed and ask how A changes. Mutants of A with slightly higher resistance cannot replace A, but mutants with slightly lower resistance can replace A. Hence A is not mutationally stable and has a direction of evolution towards smaller resistance. As in the case of one phenotype, this reduction of resistance is a very slow process and the cooperativity may randomly increase or decrease. However, here the change of cooperativity affects the ratio of the two populations. Only if the phenotype moves downwards on a vertical line in the phenospace the ratio of the two remains constant.

Second, assume that the phenotype of A is fixed and we ask how B changes. Mutants of B with a lower resistance cannot invade the population, but mutants with a slightly higher resistance can invade and replace $B$. With each mutation towards higher resistance, the population share of $B$ increases until $A$ has completely vanished. In the strict sense, both A and B evolve simultaneously. But since A evolves very slowly and B has only a relatively short way through the phenospace until A has vanished, the dominant scenario is that A does not evolve much while $B$ increases its resistance until A vanishes.

After A is lost, the evolution continues as described for the case of one phenotype. 


\subsection{CONCLUSION}

Experiments have demonstrated that two bacteria strains can coexist in an evolutionarily stable state but could not tell which phenotypes allow coexistence and how do they evolve.

To answer this, we have studied a model that is based on three realistic assumptions. First, bacteria can increase their resistance in two ways. Either they degrade the antibiotic, or they prevent the antibiotic to make its way to its target. Second, bacteria interact via the extracellular concentration. If they degrade the antibiotic, they are advantageous for other bacteria. Third, the fitness of a phenotype is determined only by its level of resistance and the extracellular concentration.

The fitness function is consistent with the observation that in an antibiotic-free environment resistant bacteria strains grow slower than not-resistant bacteria strains [100], and that the fitness is rather unaffected by small antibiotic concentrations and drops suddenly when the concentration exceeds a threshold [101, 102].

The model reproduces a number of well known phenomena in microbiology: (i) large populations resist higher concentrations than single cells, known as the inoculum effect [103, 104], (ii) the evolution of resistance in an antibiotic-polluted environment is much faster than the loss of resistance in an antibiotic-free environment [100], and (iii) an antibiotic concentration not too far above the MIC, in the range known as the "mutant selection window", strongly promotes the evolution of resistance while a concentration below the MIC does not promote and a concentration far above makes the evolution of resistance unlikely [105-107].

Our framework predicts which phenotypes can invade and coexist with the initial phenotype. Given any two coexisting phenotypes, the model also predicts which other phenotypes can invade and which of the three will disappear. In particular, a pair of coexisting strains can be replaced by a third phenotype that is much less resistant than the two coexisting strains together as shown in figure 5.5.

Besides enzymatic degradation [90, 108] one common mechanism in microbial communities is antibiotic production [109-111]. Kelsic et al. showed that in environments with complete mixing, the interplay of antibiotic production, sensitivity and degradation can lead to coexistence of three or more bacteria strains that is robust to large perturbations of species abundances and substantial differences in inherent growth rates [97]. We focus on coexistence of two bacteria strains in well-mixed environments with an external antibiotic influx. In addition, our results show that two phenotypes in an evolutionarily stable coexistence are not mutationally stable. Assuming that mutations cause a small, random step in the phenospace, the sensitive phenotype is expected to evolve a higher resistance while the resistant phe- 
notype slightly vanishes.

To conclude, we have demonstrated that the maximum degradation rate and the permeability form a pertinent phenospace for shared antibiotic resistance of bacteria strains. This contrasts exiting literature where resistance is described as a single-valued attribute [112]. This has enabled us to predict the range of phenotypes that allow two strains to coexist and to show that, although this coexistence is robust to perturbations of species abundances (i.e. evolutionarily stable), mutations will eventually move the strains to phenotypes that allow only one strain to survive. More generally, our framework provides the first step towards a theoretical foundation for understanding the phenospace, the evolutionary dynamics, the invadability and the mutational stability of cooperative antibiotic resistance, and is in agreement with experiments [88-91, 103-107].

\subsection{OUTLOOK}

First, our predictions regarding evolutionary stability, invadability and mutational stability in antibiotic resistance can be tested experimentally, since both the maximum degradation rate and the permeability are observable parameters.

In a second step, the impact of environmental fluctuations can be studied theoretically and experimentally. The antibiotic concentration in a patient who takes a daily dose of antibiotic is highly variable. What would be the effect of such fluctuations on the evolution of shared antibiotic resistance? The results in chapter 3 suggest that the evolutionarily stable states may change depending on the fluctuations. These changes could lead to a larger or smaller range of phenotypes that can coexist with each other, and thereby promote or inhibit the evolution of shared antibiotic resistance.

However, reliable predictions for fluctuating antibiotic concentrations require more detailed knowledge about the fitness functions. For constant concentrations any monotonously decreasing function $f_{*}(\mathrm{MIC})$ leads to the same results, because the concentration where the fitness functions equal does not change with the specific choice of $f_{*}$ (MIC) (see figure 5.3). In contrast, the variable concentration for which the geometric means of the fitness function are equal is sensitive to the absolute values of the fitness functions. Hence, an experimental measurement of the absolute fitness function is required before the model can be used to study the impact of fluctuating antibiotic concentrations on the evolution of antibiotic resistance.

In chapter 6 we study another biological system for which we can make predictions on the impact of fluctuating environments on the evolution, because in this system the absolute fitness functions are already partly known from direct measurements. 



\section{I INTRODUCTION}

Adaptation is often understood as an optimization process on a fitness landscape towards a local maximum. If the species is perfectly adapted to the current conditions, any change of the conditions would reduce its fitness and consequently cause an adaptation towards the new conditions until it reaches the new maximum in the fitness landscape as the stable stationary state.

However, many environmental conditions change continuously. The temperature, for example, typically changes between day and night, between the seasons of the year, with patterns lasting several years like the El Niño-Southern Oscillation, and with much longer trends like global warming and global cooling. How does a species adapt to an ever-changing environment?

Just like in chapter 3, we ask how the stationary states of the evolutionary process change depending on the environmental fluctuations. But in contrast to chapter 3, where the evolution of each species is coupled with the evolution of each other species, we assume here that the adaptation of a species to the temperature is independent of other species.

As in chapter 3, the answer depends on the time scales of the environmental changes and the adaptation rate. If the fluctuations are faster than the reproduction, the species can only adapt to live with the fluctuations by being tolerant to the range of conditions experienced during its life or by phenotypic plasticity (such as animals that change fur or feathers between winter and summer, or species that can go to specialized developmental stages to survive long times of unfavorable conditions).

If the fluctuations are much slower than the generation time, adaptation quickly reaches a current optimum and almost instantaneously follows the drift of the optimum due to the environmental change.

More challenging is the case when the fluctuations and reproduction are at a similar pace such that adaptation is always behind the changes in a non-optimal state. Naively one could expect that a good compromise is to adapt to the average of the changing condition. In this chapter we demonstrate that this is generally not correct and present a possibility to confirm this theoretical prediction. 


\subsection{OUTLINE}

In section 6.3 we present a simple theoretical example of a species whose fitness, modeled as a Gaussian function, depends on an environmental parameter, e.g. the temperature, that switches between different states or fluctuates randomly. We show that a species that is perfectly adapted to a changing environmental conditions is not perfectly adapted to the averaged environmental conditions and that these deviations depend on the kind of environmental fluctuation.

Theoretically, all species that adapt to changing environments can be subject to such deviations. The following sections are a first step towards empiric evidence for the predicted deviations using the temperature adaptation of nematodes. Nematodes, in particular Caenorhabditis elegans, qualify for this aim for several reasons. They are well studied regarding their temperature dependent development, strains from different habitats exist that are adapted to different temperature conditions, and nematodes reproduce fast enough to measure in reasonable time how their fitness depends on the temperature.

In section 6.4 we develop a model for the temperature-dependent development and reproduction of nematodes based on existing datasets. We compare the results of the model with existing experimental data for constant temperatures and with data from experiments with variable temperatures that were conducted to test the model.

In the next step, we use this model to predict how nematodes adapt to realistic temperature scenarios. To generate such realistic temperature scenarios, we take historical temperature records of weather stations on Reunion island, described in section 6.5, and use these air temperatures to estimate soil temperatures, described in section 6.6. From the simulations of the model we find the phenotypes that would be best adapted to a specific temperature scenario and we can compare it with the phenotype that would be best adapted to the average temperature of this scenario. The predicted deviations between these two phenotypes are presented in section 6.7.

To conclude, we discuss in section 6.8 how the predicted deviations can be tested experimentally.

\subsection{THEORETICAL EXAMPLES}

\subsubsection{Periodically changing temperature}

Consider a species that reproduces exponentially with a growth rate $r(T)$, which depends on the temperature $T$. It has a maximum growth rate $r\left(T_{\mathrm{id}}\right)=r_{\max }$ at an ideal temperature $T_{\mathrm{id}}$ and a smaller growth rate the further the temperature deviates from the ideal temperature. In general, deviations to high or low temperatures may affect the 
fitness differently (as we will see in chapter 6.4), but for this simple example we assume a symmetric Gaussian fitness function

$$
f\left(T ; T_{\text {id }}\right)=f_{\text {id }} \exp \left(-\frac{\left(T-T_{\text {id }}\right)^{2}}{2 \sigma^{2}}\right),
$$

as depicted in figure 6.1A.

In a constant temperature $T \neq T_{\mathrm{id}}$, a mutant has an advantage if its ideal temperature $T_{\text {id }}^{\prime}$ is closer to the environmental temperature than the ideal temperature $T_{\mathrm{id}}$ of the other individuals. In the long-term such mutations replace the individuals of the original phenotype and the species adapts its ideal temperature to the environmental temperature such that $T_{\text {id }}=T$.

What happens if the environmental temperature is changing? For our simple example we assume that the temperature switches between $T_{1}$ and $T_{2}$ with duration $0 \leq p \leq 1$ at $T_{1}$ and $q=1-p$ at $T_{2}$, as shown in figure 6.1B.

We analyze the situation in a simple exponential growth process, first in the discrete time variant, which is the common model for synchronously reproducing species (e.g. annual plants), and second in the continuous time variant, which is the common model for asynchronously reproducing species (e.g. bacteria).

In the case of discrete time, the size of the population $n_{t}$ at time step $t \in \mathbb{N}$ can be described as

$$
n_{t}=r_{t} n_{t-1}=\left(1+f\left(T_{t} ; T_{\mathrm{id}}\right)\right) n_{t-1} .
$$

With a constant growth rate we can compute the size of the population at time $t$ as $n_{t}=r^{t} n_{0}$. With a varying growth rate, the population at time $t$ is

$$
\begin{aligned}
n_{t} & =n_{0} \prod_{t^{\prime}=1}^{t}\left(1+f\left(T_{t^{\prime}} ; T_{\mathrm{id}}\right)\right) \\
& =n_{0}{\overline{\left(1+f\left(T_{t} ; T_{\mathrm{id}}\right)\right)}}^{t},
\end{aligned}
$$

where $T_{t}$ is the environmental temperature at time step $t$.

In the case of continuous time, the growth of the population size $n$ can be described as

$$
\frac{\mathrm{d} n}{\mathrm{~d} t}=r_{t} n=f\left(T(t) ; T_{\mathrm{id}}\right) \cdot n
$$

With a constant growth rate $r$ the size of the population at time $t$ is $n(t)=n_{0} e^{r t}$, and with a varying growth rate the size of the population at time $t$ is

$$
n(t)=n_{0} \exp \left(\int_{\tilde{t}=0}^{t} f\left(T(\tilde{t}) ; T_{\mathrm{id}}\right) d \tilde{t}\right) .
$$


If $T(t)$ is a periodic function with period $\tau$ and we evaluate $n(t)$ only at multiples of the period, we can write this as

$$
n(k \tau)=n_{0} \exp (\langle f\rangle \cdot k \tau),
$$

where $k \in \mathbb{N}$ and $\langle f\rangle$ denotes the arithmetic average of $f$.

In general, the time-average of the growth rate is the geometric average of the growth rate $r_{\text {discrete }}=(1+f)$ in the case of discrete time and the arithmetic average of $r_{\text {continuous }}=f$ in the case of continuous time. In the special case of our example with a temperature switching between two values, the time-averaged growth rates are

$$
\begin{aligned}
r_{\text {discrete }}\left(T_{1}, T_{2}, p ; T_{\mathrm{id}}\right) & =\overline{(1+f)} \\
& =\left(1+f\left(T_{1} ; T_{\mathrm{id}}\right)\right)^{p} \cdot\left(1+f\left(T_{2} ; T_{\mathrm{id}}\right)\right)^{(1-p)} \\
r_{\text {continuous }}\left(T_{1}, T_{2}, p ; T_{\mathrm{id}}\right) & =\langle f\rangle \\
& =p f\left(T_{1} ; T_{\mathrm{id}}\right)+(1-p) f\left(T_{2} ; T_{\mathrm{id}}\right)
\end{aligned}
$$

Adaptation changes the phenotype $T_{\mathrm{id}}$ to maximize these time-averaged growth rates for the specific temperature fluctuation. Figure 6.2 shows for two examples ( $p=0.25$ and $p=0.5$ ) how the time-averaged growth rates depend on the phenotype $T_{\mathrm{id}}$. In the case of unequal durations $(p=0.25)$, the maximal growth rate deviates to the same direction in both continuous time and discrete time growth model. In the case of equal durations $(p=0.5)$, the position of the maximum matches the average temperature in the discrete time growth model and in the continuous time growth model there is a local minimum, an unstable fixed point of adaptation, at the average temperature. It can be shown analytically that

$$
\left.\frac{\mathrm{d} r_{\text {discrete }}}{\mathrm{d} T_{\text {id }}}\right|_{\langle T\rangle}=\left.\frac{\mathrm{d} r_{\text {continuous }}}{\mathrm{d} T_{\text {id }}}\right|_{\langle T\rangle}=0 \text { if } p \in\left\{0, \frac{1}{2}, 1\right\}
$$

and

$$
\left.\frac{\mathrm{d}^{2} r_{\text {discrete }}}{\mathrm{d} T_{\text {id }}^{2}}\right|_{\langle T\rangle}=\left.\frac{\mathrm{d}^{2} r_{\text {continuous }}}{\mathrm{d} T_{\text {id }}^{2}}\right|_{\langle T\rangle}=-\frac{f_{\text {id }}}{\sigma^{2}} \text { if } p \in\{0,1\} .
$$

Hence, $T_{\mathrm{id}}=\langle T\rangle$ is a stable fixed point for $p=0$ and $p=1$ and a stable or unstable fixed point for $p=0.5$. For other values of $p$, the fitness maximum may deviate from the average temperature $\langle T\rangle$, as shown in figure 6.3.

The different stability of the fixed point in the discrete time model and the continuous time model using the same parameters, as shown in the example in figure $6.2 \mathrm{~B}$ with $p=0.5$, is caused by the different impact of small and large numbers on the geometric and the arithmetic mean. Consider the two Gaussian fitness functions $f_{1}=f\left(T_{1} ; T_{\mathrm{id}}\right)$ and $f_{2}=f\left(T_{2} ; T_{\mathrm{id}}\right)$ with a maximum at $T_{1}=T_{\mathrm{id}}$ and at $T_{2}=T_{\mathrm{id}}$, 

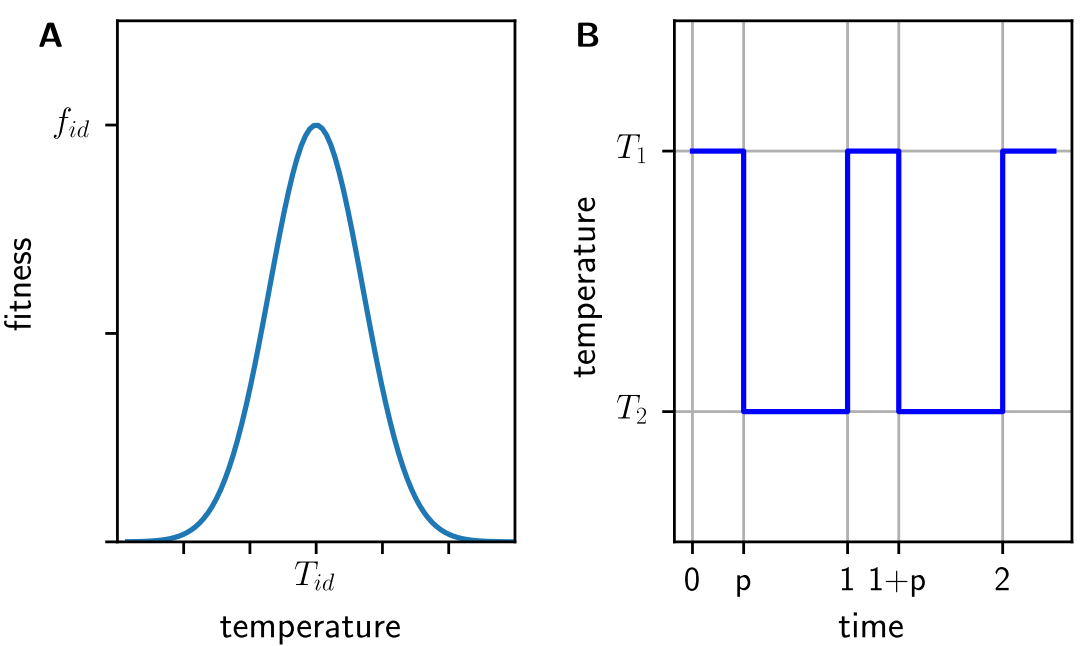

Figure 6.1: Simple example for adaptation to variable temperatures. The fitness is modeled as a Gaussian (A) and the temperature switches between $T_{1}$ and $T_{2}$ with durations $p$ and $q=1-p$ (B).

respectively. If the Gaussian functions do not have much overlap, the arithmetic mean of $f_{1}$ and $f_{2}$ has two peaks, one where $f_{1}$ has its maximum and another one where $f_{2}$ has its maximum. In contrast, the geometric mean of $f_{1}$ and $f_{2}$ has a small value at these positions, because the maximum of $f_{1}$ coincides with a low value of $f_{2}$ and vice versa due to the small overlap of $f_{1}$ and $f_{2}$. The geometric mean of $f_{1}$ and $f_{2}$ has its maximum where the product of the two functions is maximal, which is in this case the position of the largest overlap of the two functions. Hence, the discrete time model, where the timeaveraged fitness is given by the geometric mean, has a stable fixed point and the continuous time model, where the time-averaged fitness is given by the arithmetic mean, has an unstable fixed point at the position of the largest overlap, $T_{\mathrm{id}}=\langle T\rangle$. For an illustration of this effect see figure 6.4.

\subsubsection{Randomly fluctuating temperature}

The calculation of time-averaged growth rates with randomly fluctuating temperatures is conceptually slightly different, but leads to similar results.

We assume that the temperature is a random variable $T$ with a probability density function $\operatorname{pdf}(T)$. In order to calculate the time-averaged growth for the continuous time case (analogous to equation 6.9), we 

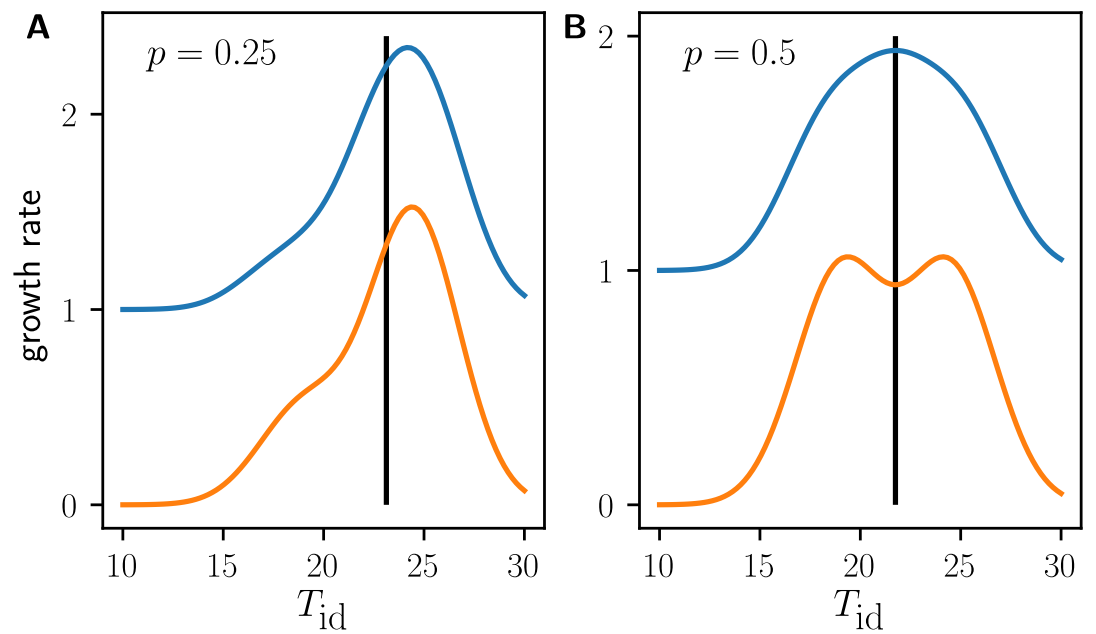

Figure 6.2: Time-averaged growth rates resulting from the fitness function and temperature fluctuation depicted in figure 6.1. (A) shows the result from a temperature fluctuation with unequal durations and (B) with equal durations. The vertical black lines show the average temperatures, the blue curves are time-averaged growth rates in the discrete-time case (equation 6.8) and the orange curves are the time-averaged growth rates in the the continuoustime case (equation 6.9). The parameters of the Gaussian fitness function are $\sigma^{2}=5$ and $f_{\text {id }}=2$. The temperature switches between $T_{1}=19$ and $T_{2}=24.5$ with the duration $p=0.25$ in (A) and $p=0.5$ in (B).

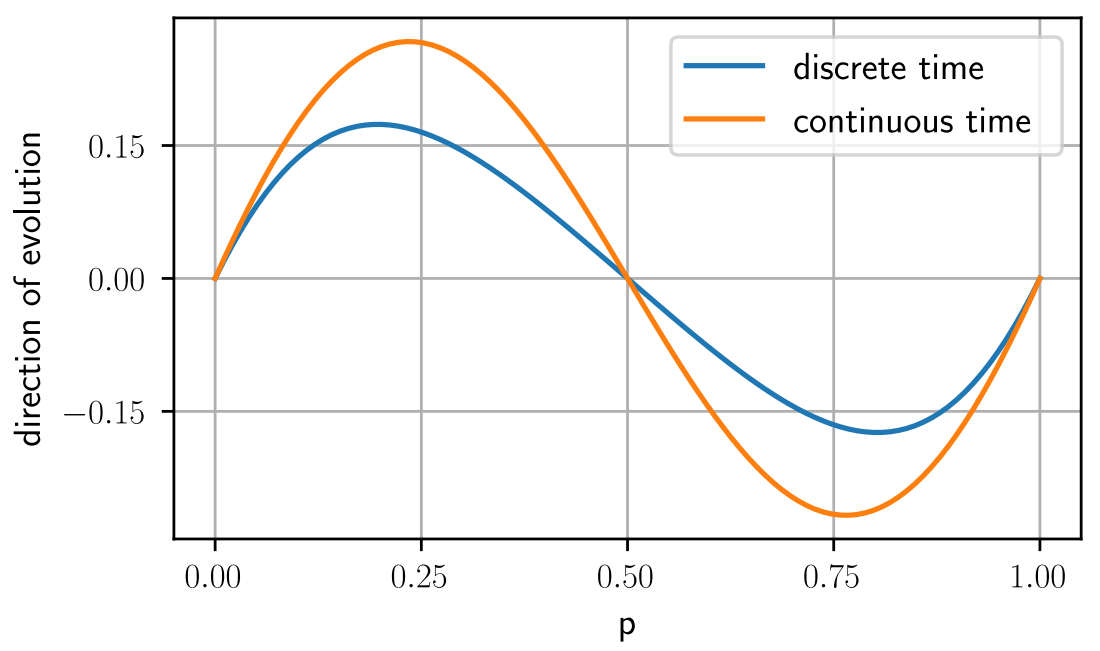

Figure 6.3: Direction of evolution at average temperature. The two curves show $\left.\frac{\mathrm{d} r}{\mathrm{~d} T_{\mathrm{id}}}\right|_{\langle T\rangle}$ for the discrete and continuous time growth model with the same parameters as in figure 6.2. Positive/negative values indicate that the fitness maximum is shifted to the right/left with respect to the average temperature. 

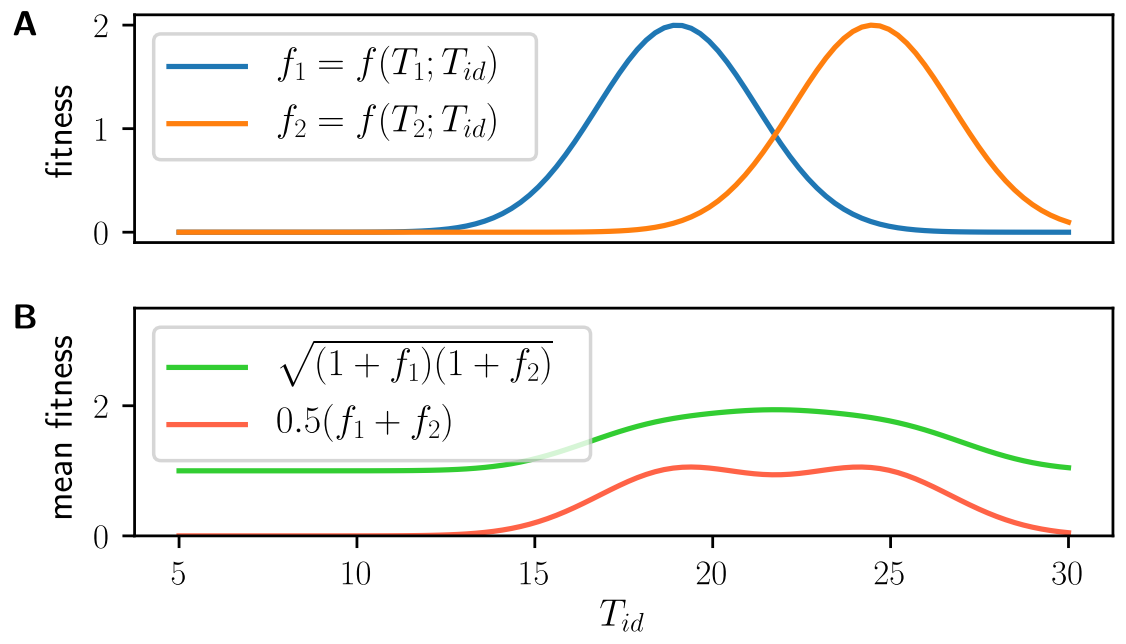

Figure 6.4: Effect of geometric and arithmetic mean on the stability of the fixed point of adaptation. The two curves in (A) show the fitness at temperature $T_{1}$ and $T_{2}$. The two curves in (B) show the arithmetic and the geometric mean of the two curves in (A).

can write the arithmetic average as an integral over the probability density function

$$
\begin{aligned}
r_{\text {continuous }}\left(\operatorname{pdf}(T) ; T_{\text {id }}\right) & =\langle f\rangle \\
& =\int_{-\infty}^{\infty} f(T) \operatorname{pdf}(T) \mathrm{d} T .
\end{aligned}
$$

In order to calculate the time-averaged growth rate for the discrete time case (analogous to equation 6.8), we need to compute the geometric mean integrated over the probability density function $\operatorname{pdf}(T)$. The most convenient way to express this is the product-integral [113, 114].

$$
\begin{aligned}
& r_{\text {discrete }}\left(T_{\text {id }}\right)=\overline{(1+f)} \\
& =\stackrel{\mathcal{P}}{\infty}_{-\infty}^{\infty}(1+f)^{\operatorname{pdf}(T) \mathrm{d} T} \\
& =\exp \left(\int_{-\infty}^{\infty} \operatorname{pdf}(T) \log (1+f) \mathrm{d} T\right)
\end{aligned}
$$

Just like the usual integral is the continuous limit of the sum, the product-integral is the continuous limit of a product, here denoted ${ }^{1}$ by $\mathcal{P}$. By taking the logarithm the geometric mean can be trans-

I Although the product-integral has already a long history in mathematical biology going back at least to Vito Volterra, there is not yet an agreement on which symbol to use. Common forms are $\int g(x)^{\mathrm{d} x}$ and $\prod g(x)^{\mathrm{d} x}$. Here we use $\mathcal{P} g(x)^{\mathrm{d} x}$ following $\mathrm{N}$. Arley [115], because this form is consistent with using capital Greek letters $\Sigma$ and $\Pi$ for discrete sums and discrete products and the stylized capital Latin letters $S$ and $P$ for their integral counterpart. 
formed to the slightly more complex form using only the sum-based integral.

For an example we use again a Gaussian fitness function as defined in equation 6.I with $\sigma^{2}=2, f_{\text {id }}=2$ and the ideal temperature $T_{\text {id }}$ that is subject to adaptation. As the probability density function of the temperature we take the skew normal distribution $\operatorname{pdf}(\xi, \omega, \alpha)$, where $\xi$ determines the location, $\omega$ the width and $\alpha$ the skewness of the distribution [116]. With zero skewness $(\alpha=0)$ the skew normal distribution equals the normal distribution with variance $\sigma^{2}=\omega^{2}$ and location $\mu=\xi$. Figure 6.5 shows three distributions with different skewness values and the corresponding numerical solution of equation 6.13 and 6.16. Similar to the unequal durations $p$ and $1-p$ for the two temperatures in section 6.3.1, the skewness results in a deviation of the fitness maximum from the average temperatures. Consequently, a phenotype with the ideal temperature $T_{\mathrm{id}}=\langle T\rangle$ is subject to an adaptive pressure and evolves towards higher temperatures if the probability density of the random temperature is right-skewed and towards lower temperatures if the probability density is left-skewed, as shown in figure 6.6.

\subsection{MOdeling THE GROWTH OF NEMATOdES}

\subsubsection{Development}

The normal development of nematodes consists of four stages (also known as molts) that each end with a lethargus phase. Some time after the fourth-molt lethargus the nematode starts laying eggs for a limited period of time. To survive unfavorable conditions nematodes may develop into an alternative third stage known as the "dauer stage", in which it can survive long times of unfavorable conditions [117]. If the conditions become better the dauer larvae can continue their development to adults.

Here we aim to model the temperature dependence of the normal development and assume that, besides the temperature, all conditions are favorable enough that the nematodes do not develop to the dauer stage.

Experiments show that the duration of the normal developmental stages changes substantially with the temperature. Figure 6.7A shows the mean ages at these stages observed in cultures of $C$. elegans strain N2 at three different temperatures [118]. However, if we look at the relative durations, e.g. by dividing all ages by the age at which the nematodes stop laying eggs as shown in figure $6.7 \mathrm{~B}$, these relative ages are almost independent of the temperature. This suggests that the temperature does not affect the development except for the time a nematode needs to progress in its development. To formalize this idea, we introduce a biological age $a$ that corresponds to the devel- 

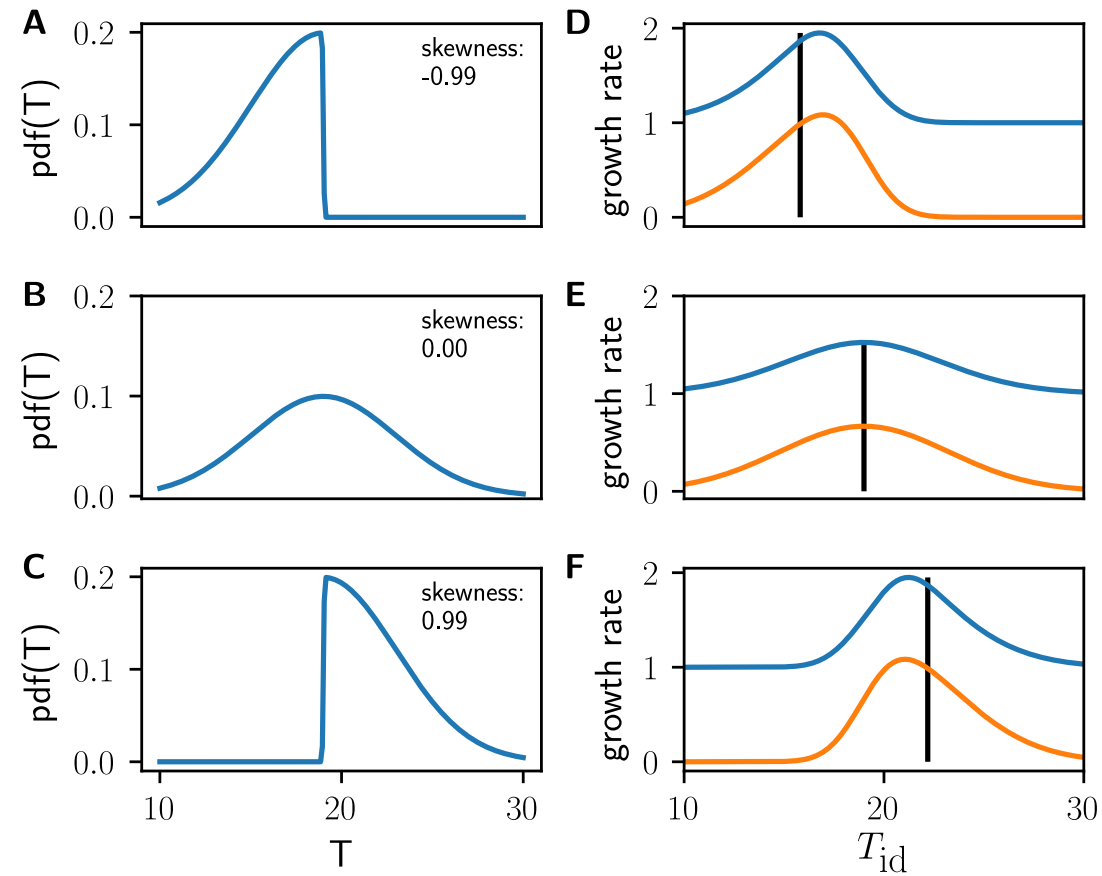

Figure 6.5: Time-averaged growth rates resulting from randomly fluctuating temperatures. The fitness is modeled as a Gaussian as defined in equation 6.1 with $\sigma^{2}=2$ and $f_{\mathrm{id}}=2$. (A), (B) and (C) show a left-skewed, symmetric, and right-skewed probability density function. In (D), (E) and (F), the vertical black lines show the average temperatures $\langle T\rangle$, the blue curves show the time-averaged growth rate in the discrete-time variant (equation 6.16) and the orange curve shows the time-averaged growth rate in the continuous-time variant (equation 6.13).

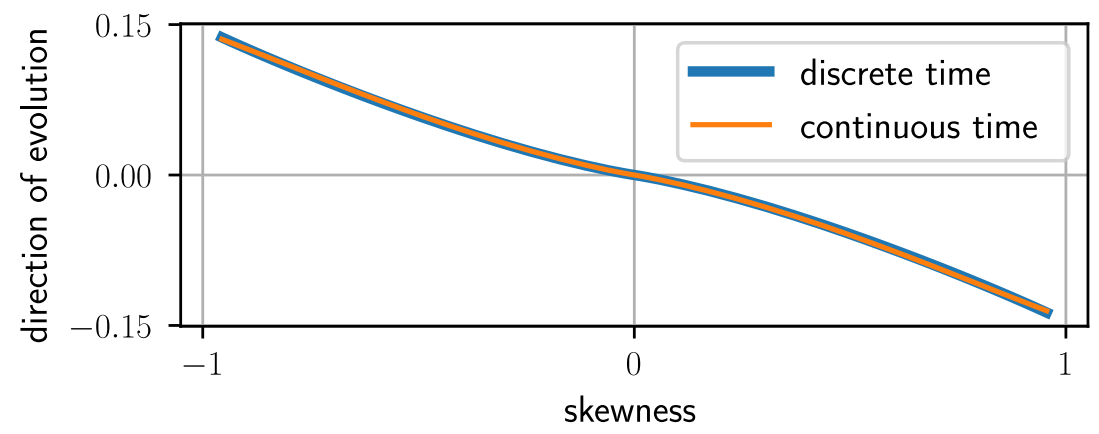

Figure 6.6: Direction of evolution when the ideal temperature equals the average temperature. The two curves show $\left.\frac{\mathrm{d} r}{\mathrm{~d} T_{\mathrm{id}}}\right|_{\langle T\rangle}$ for the discrete and continuous time growth model depending on the skewness of the probability density $\operatorname{pdf}(T)$. Positive/negative values indicate that the fitness maximum is shifted to the right/left with respect to the average temperature. 


\begin{tabular}{l|c} 
developmental stage & biological age \\
\hline \hline Egg laid & 0 \\
Egg hatches & 9.23 \\
First-molt lethargus & 20.35 \\
Second-molt lethargus & 27.53 \\
Third-molt lethargus & 34.18 \\
Fourth-molt lethargus & 43.24 \\
Egg-laying begins & 51.40 \\
Egg-laying ends & 100 \\
Death & 282.20
\end{tabular}

Table 6.1: Temperature-independent biological ages at developmental stages of C. elegans.

opmental progress and an ageing rate $\frac{\mathrm{d} a}{\mathrm{~d} t}(T)$ that depends on the temperature $T$. The ageing rate determines how much the nematode ages per hour in real time. We define the biological age at 'Egg-laying ends' as $a:=100$ and the ageing rate as

$$
\begin{aligned}
\frac{\mathrm{d} a}{\mathrm{~d} t}(T) & =\frac{100}{\operatorname{ele}(T)} \\
& \approx \frac{100}{0.843 \cdot T^{2}-44.774 \cdot T+680.641},
\end{aligned}
$$

where ele $(T)$ is a quadratic function fitted to the 'Egg-laying ends'data in figure $6.7 \mathrm{~A}$. We define the biological ages of the developmental stages as the average of the relative ages obtained from the data multiplied with 100 to scale biological age of 'Egg-laying ends' to 100. These averages are shown as vertical lines in figure 6.7B.

The resulting values are listed in table 6.1. Since the dataset does not include the ages at which the nematodes died, this value was calculated using an additional data set (table 1 in [119], strain N2).

\subsubsection{Reproduction}

The biological ages in table 6.1 define when the individuals in our model begin and when they stop laying eggs. In order to model the population growth we further need to define an egg-laying rate. For C. elegans strain N2, the observed data in Figure 6.8 show how the total number of eggs depends on the temperature and, for three temperatures, how the egg-laying rate depends on the age. From these two observations, we aim to infer the egg-laying rate $r_{\text {eggs }}(T, a)$ that depends on the temperature and the biological age.

The total number of eggs produced by one nematode depending on the temperature is shown in figure 6.8A. Since the number of eggs 

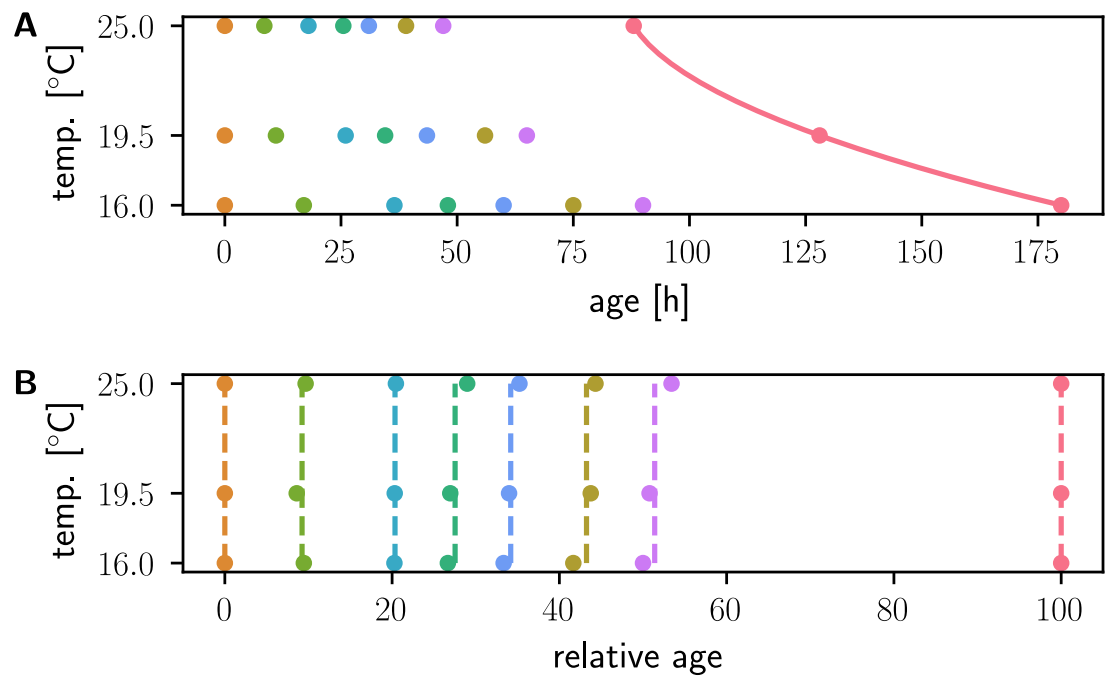

\begin{tabular}{|lll|}
\hline Egg laid & - Third-molt lethargus \\
- Egg hatches & - Fourth-molt lethargus \\
- First-molt lethargus & $\bullet$ Egg-laying begins \\
\hline
\end{tabular}

Figure 6.7: Development of C. elegans at different temperatures. The dots in (A) show the mean age of a nematode in hours from when the egg was laid at the developmental stages for three different temperatures (data from table 2 in [118]). The green curve is a fit of a quadratic function to the ages at 'Egg-laying ends' which is used to define the ageing rate (see main text). (B) shows the same data points but with the ages relative to the age at 'Egglaying ends'. The dashed vertical lines are at the mean age of the three corresponding points, which define the biological ages of the developmental stages (see main text). 
varies a lot between individuals, we model the total number of eggs as a random variable. We use two quadratic functions $f_{1}(T)$ and $f_{2}(T)$ fitted to the upper and lower values of the data for each temperature (see figure 6.8A) and define the probability density $P\left(N_{\text {eggs }}, T\right)$ of the lifetime fecundity as a uniform distribution between the upper and lower fit (restricted to positive values)

$$
P\left(N_{\text {eggs }}, T\right)=\mathcal{U}\left(\max \left\{0, f_{1}(T)\right\}, \max \left\{0, f_{2}(T)\right\}\right),
$$

where $f_{1}(T)=-4.64 \cdot T^{2}+169.06 \cdot T-1136.63$ and $f_{2}(T)=-2.92$. $T^{2}+105.85 \cdot T-734.95$.

The egg-laying rate depending on the age is shown in figure 6.8B. At all three temperatures the rate reaches a maximum approximately in the middle of the egg-laying period. We model the rate with a simple piecewise linear function that is zero below the biological age when egg-laying begins $a_{1}=a_{\text {Egg-laying begins }}$ and above the biological age when egg-laying ends $a_{2}=a_{\text {Egg-laying ends }}$ as defined in table 6.I, and has a temperature-dependent maximum $r_{\text {eggs }}^{\max }(T)$ in the middle of this period,

$$
r_{\mathrm{eggs}}(T, a)=\left\{\begin{array}{ll}
0 & \text { if } a<a_{1} \\
\frac{\left(a-a_{1}\right) r_{\mathrm{eggs}} \max (T)}{0.5\left(a_{2}-a_{1}\right)} & \text { if } a_{1} \leq a<\frac{a_{2}-a_{1}}{2} \\
r_{\mathrm{eggs}}^{\max }(T)-\frac{\left(a-0.5\left(a_{2}+a_{1}\right)\right) r_{\mathrm{eggs}}^{\max }(T)}{0.5\left(a_{2}-a_{1}\right)} & \text { if } \frac{a_{2}-a_{1}}{2} \leq a \leq a_{2} \\
0 & \text { if } a>a_{2}
\end{array} .\right.
$$

Now we choose the maximum egg-laying rate $r_{\text {eggs }}^{\max }(T)$ such that the integral of the egg-laying rate function equals the lifetime fecundity.

$$
\begin{aligned}
N_{\text {eggs }}(T) & =\int_{0}^{\infty} r_{\mathrm{eggs}}(T, a) \mathrm{d} a \\
& =\frac{1}{2} r_{\mathrm{eggs}}^{\max }(T)\left(a_{2}-a_{1}\right) \\
& \Rightarrow r_{\mathrm{eggs}}^{\max }(T)=\frac{2 \cdot N_{\mathrm{eggs}}(T)}{a_{2}-a_{1}}
\end{aligned}
$$

The egg-laying rate function $r_{\text {eggs }}(T, a)$ is shown for three temperatures and an average lifetime fecundity $\left\langle N_{\text {eggs }}(T)\right\rangle$ as solid lines in figure $6.8 \mathrm{~B}$ (with the biological age translated to real time to allow comparison with the data). For $16^{\circ} \mathrm{C}$ and $20^{\circ} \mathrm{C}$ the model rate exceeds the data and for $25^{\circ} \mathrm{C}$ the data exceeds the model rate. However, this deviation is within the typical variation of the lifetime fecundity seen in (A), where the three red squares mark the lifetime fecundity of the data shown in (B). 

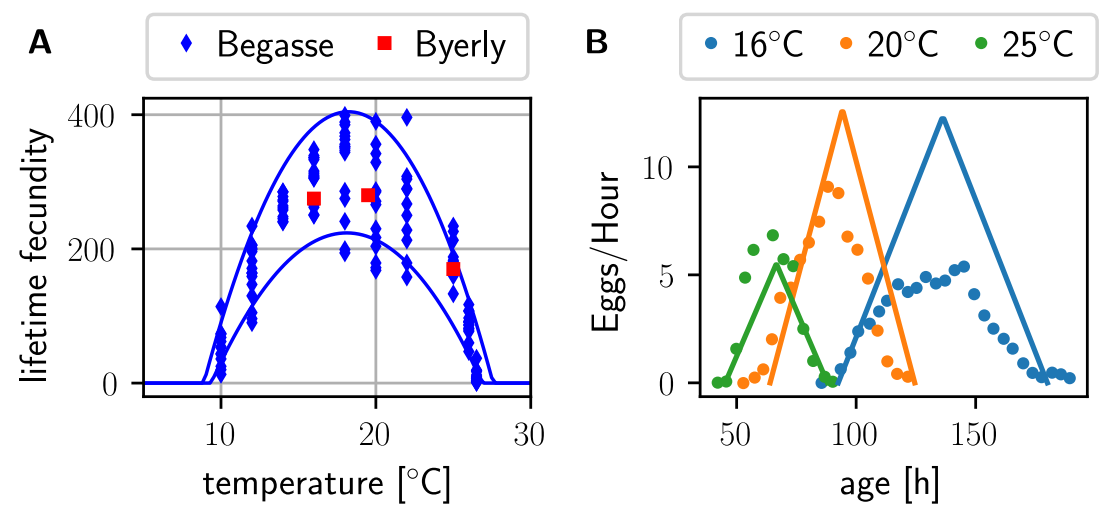

Figure 6.8: Reproduction of C. elegans at different temperatures. (A) shows the total number of eggs produced by one nematode at different temperatures. The data set named "Begasse" is published in [120], the raw data for reproducing the figure were provided by Dr. Mark Leaver. The data set named "Byerly" is taken from table 2 of [118]. The two blue curves are quadratic functions fitted to the upper and lower values for each temperature in the data set "Begasse" (set to zero where these quadratic functions have negative values, see equation 6.19). (B) shows the rate of eggs laid by one nematode at three different temperatures (data extracted from figure 8 in [118]). The solid lines show the piecewise linear model for the egg-laying rate explained in the main text (equation 6.20).

\subsubsection{Population growth}

With the ageing rate from section 6.4.1 and the egg-laying rate from section 6.4.2 we could set up an agent-based simulation to model a set of individuals that develop and reproduce. However, the population size that can be simulated in such an agent-based simulation is very limited because the number of calculations per time step and the required memory increase with the number of individuals.

We need a method to simulate large populations, because in section 6.7 we want to use this model to measure the average growth rate in realistic temperature conditions. Since a realistic temperature scenario has a period of one year, we need to simulate the growth of a population for at least one full year. In such long time intervals the populations reach a size where an agent-based simulation is practically impossible.

Instead of an agent-based model, we discretize the age and solve a system of equations that describe how the age distribution of the population evolves in time. The discrete age is defined as $a_{i}=i \alpha$, where $\alpha$ is a small constant (the age increment) and $i \in \mathbb{N}$. In addition, we discretize the uniform distribution for the lifetime fecundity (equation 6.19) in equidistant steps $N_{\text {eggs }, j}(T)$ between the minimal lifetime fecundity $(j=0)$ and the maximal lifetime fecundity $(j=m)$. 
Now we can describe the population by an age distribution defined as the number of individuals $n(i, j)$ at age $a_{i}$ and with lifetime fecundity $N_{\text {eggs }, j}(T)$, such that the total population size is $N=\sum_{i=0}^{\infty} \sum_{j=0}^{m} n(i, j)$. With every time step from $t$ to $t+\alpha\left(\frac{\mathrm{d} a}{\mathrm{~d} t}(T)\right)^{-1}$, where $\frac{\mathrm{d} a}{\mathrm{~d} t}(T)$ is the temperature-dependent ageing rate defined in equation 6.18, the age of all individuals increases by $\alpha$. During this step the distribution $n(i, j)$ changes by two processes, each individual gets one step older and some individuals will produce eggs that appear in the distribution after the step $n^{\prime}(0, j)$ at age $a_{0}$ and with a random fecundity phenotype $j$. These changes can be written as

$$
\begin{aligned}
& n^{\prime}(i, j)=n(i-1, j) \\
& n^{\prime}(0, j)=\sum_{k=1}^{E} \delta_{j, e_{k^{\prime}}}
\end{aligned}
$$

where $E=\left\lfloor\alpha \sum_{j=0}^{m} \sum_{i=0}^{\infty} r_{\mathrm{eggs}, j}(T, i \alpha) \cdot n(i, j)\right\rfloor$ is the number of new eggs, $r_{\text {eggs }, j}(T, i \alpha)$ is the egg-laying-rate corresponding to the lifetime fecundity $N_{\text {eggs,j }}(T)$ (as defined in equation 6.20), $\delta$ is the Kronecker delta, and $e_{k}$ is a random number for each new egg $k$ drawn from the discrete uniform distribution $\mathcal{U}(0, m)$ to determine the fecundity phenotype of the new eggs. The initial condition at time $t=0$ can be any distribution $n(i, j)$ and the boundary conditions at any time $t$ are $n(i, j)=0$ for all $i<0$ and $n(i, j)=0$ for all $i>\left\lfloor\frac{a_{\text {Death }}}{\alpha}\right\rfloor$ (with the value $a_{\text {Death }}$ as given in table 6.1).

\subsubsection{Growth rates at constant temperatures}

As a first test how the model works we can use it to measure the growth rates at constant temperatures and compare it with experimental data.

First, we need to define two different growth rates that we measure. If the temperature is constant and in the range where each nematode produces at least one egg during its life, the long-term growth can be approximated by an exponential growth. In this case we measure the growth rate $r$ of an exponential growth process $N(t)=N_{0} e^{r t}$ that approximates the long-term growth obtained from the simulated population. If the constant temperature does not allow the nematodes to reproduce, the population declines with a certain percentage per time, because the population ages with a constant ageing rate, the old individuals die and no new eggs are produced. In this case we define $r^{\prime}$ as the growth rate with which the function $N(t)=N_{0}\left(1+r^{\prime} t\right)$ approximates the decline of the simulated population.

Figure 6.9A shows some example realizations for growth and decline with different constant temperatures. The simulations with temperatures that allow growth started with one initial individual at age 
0 and the simulations with temperatures that result in a declining population started with a homogeneous age distribution of 1000 individuals. The curve for $15^{\circ} \mathrm{C}$ shows a sudden increase after four days, indicating that the starter nematode begins laying eggs. The curve for $20^{\circ} \mathrm{C}$ has a second increase after six days when the first offspring of the starter begin laying eggs. The curve for $25^{\circ} \mathrm{C}$ includes even a third generation of offspring. The curves of the declining populations are just straight lines in these examples, which is a result of the initially homogeneous age distribution.

Figure 6.9B shows the approximated growth rates depending on the temperature.

Note that the ageing rate between $16^{\circ} \mathrm{C}$ and $25^{\circ} \mathrm{C}$ is an interpolation of the available data (figure 6.7), while outside this range it is an extrapolation. This extrapolation is certainly incorrect for temperatures above $28^{\circ} \mathrm{C}$, because higher temperatures are harmful to $\mathrm{C}$. elegans strain N2 [119]. However, this does not affect the resulting growth rates because the lifetime fecundity is already zero at $27.15^{\circ} \mathrm{C}$. The extrapolation for low temperatures below $16^{\circ} \mathrm{C}$ is perhaps also not perfect, but low temperatures (above $0^{\circ} \mathrm{C}$ ) are not harmful and also the slowdown of the cell division process can be well extrapolated from temperatures between $10^{\circ} \mathrm{C}$ and $20^{\circ} \mathrm{C}$ [120]. For the same reason as for high temperatures, an incorrect extrapolation of the ageing rate below $9.18{ }^{\circ} \mathrm{C}$ does not corrupt the predicted growth rates.

\subsubsection{Growth rates with switching temperatures}

The model is designed based on data that were measured at constant temperatures and as shown in the previous chapter the predicted growth rates for constant temperatures agree well with experimental results. Can we use the model for changing temperatures as well? Do nematodes behave at a current temperature as if it were a constant temperature or are temperature changes stressful for them?

In order to test how the nematodes react to changing temperatures it is necessary to do experiments long enough to get more than one generation. On the other hand, since the populations grow exponentially, it is difficult to do more than two generations because the nematodes need to have always enough food and space and they need to be counted several times during the experiments. As a compromise, we decided to do one experiment repeated six times with relatively strong and therefore potentially stressful temperature changes between $13^{\circ} \mathrm{C}$ and $25^{\circ} \mathrm{C}$. These two temperatures switch instantaneously every 12 hours during 8 days.

The results are shown in figure 6.10. In general, the results follow the shape of the predicted growth curve with the bumps that indicate the generations. For the last measurement after 8 days we have only two data points, because the others are still to be analyzed. These two data 
$\mathbf{A}$
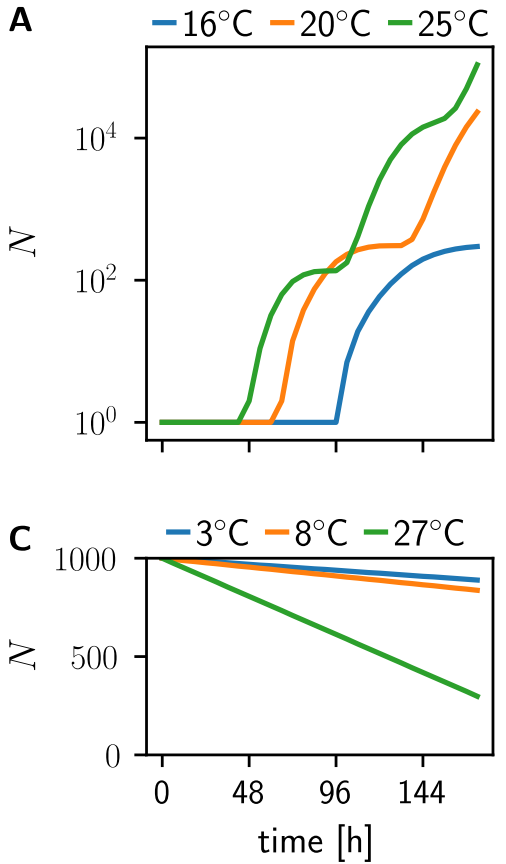
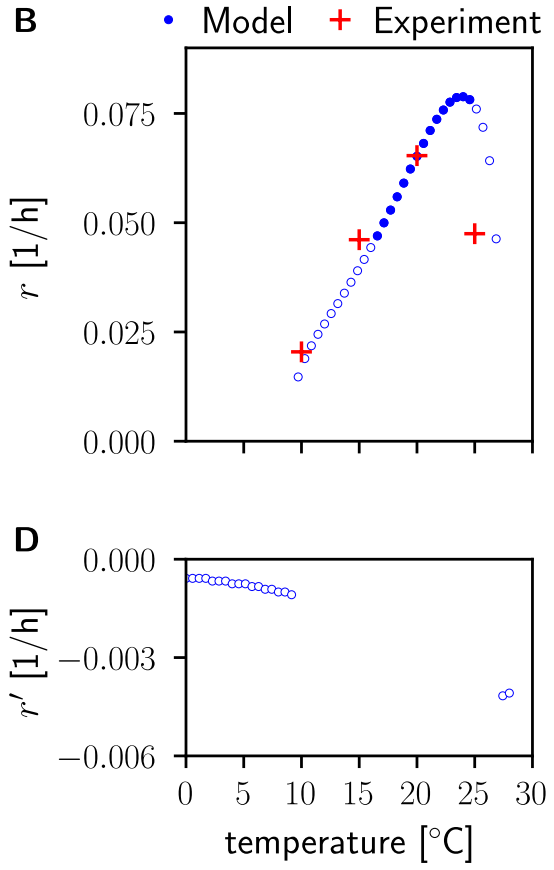

Figure 6.9: Population growth with constant temperatures. (A) shows three examples of growing populations (starting from one nematode of age 0 ) as predicted by the model for temperatures which allow reproduction. (C) shows declining populations (starting from 1000 nematodes of a homogeneous age distribution) as predicted by the model for temperatures which do not allow reproduction. (B) and (D) show the growth rates that approximate the simulated long-term population growth. Filled circles indicate that the temperature is in the range where the ageing rate is an interpolation of the data $\left(16^{\circ} \mathrm{C}\right.$ to $\left.25^{\circ} \mathrm{C}\right)$ and empty circles indicate that the temperature is in the range where the ageing rate is an extrapolation of the data. The red crosses are experimental data from Venette and Ferris [121]. In (B), $r$ is the growth rate which approximates the long-term population growth with $N(t)=N_{0} e^{r t}$. In (D), $r^{\prime}$ is the growth rate which approximates the population growth with $N(t)=N_{0}\left(1+r^{\prime} t\right)$. 


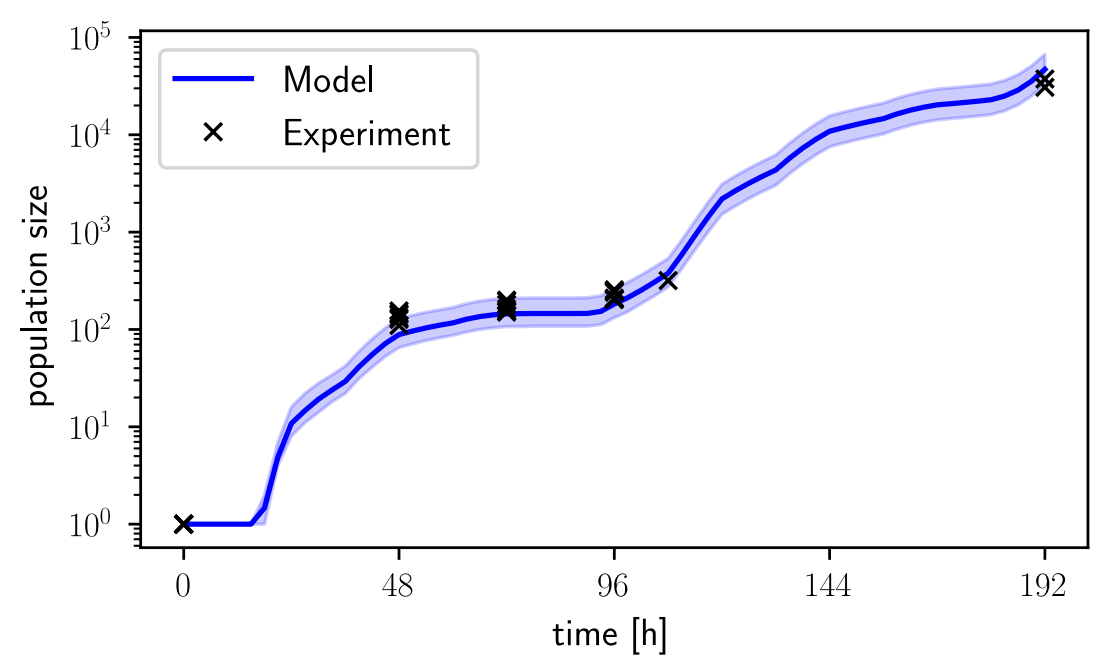

Figure 6.10: Population growth with switching temperatures. The blue line shows the expectation value and the blue area shows the $95 \%$ confidence interval of the population size based on the model. The black crosses show the experimental results. The initial population in the model and the experiments consists of one adult nematode. The temperature switches between $13^{\circ} \mathrm{C}$ and $25^{\circ} \mathrm{C}$ every 12 hours for 8 days. The experiment was repeated six times, but at day 8 only two data points are shown because the analysis is not yet completed. The experiments were done by Dr. Mark Leaver (Biotechnology Center (BIOTEC), TU Dresden) using C. elegans strain N2.

points are about $50 \%$ below the predicted population size. More data points are necessary to conclude whether the results are scattered or show a systematic deviation between model and experiments. Possible reasons for a systematic deviation are that the nematodes had not always enough food or space, that the counting is incomplete (in particular counting the eggs is difficult due to their small size), and that the model overestimates the growth because it does not include any negative effect on the growth rate due to stress caused by temperature fluctuations.

\subsection{WEATHER DATA}

In order to measure the long-term growth rates in realistic temperature conditions using the model described in section 6.4, we need one year long records of the temperature with a temporal resolution that includes the daily fluctuations. Since one year can substantially deviate from another year, the results should ideally be averaged over several years. Since the results are going to be compared to experimentally measured growth rates using nematode strains that were collected from different locations on Reunion island, we need temper- 
ature records from several regions on Reunion island.

The national meteorological institute of France "Météo-France" operates a number of weather stations on Reunion island. Only summaries of the data are published, but the raw data can be purchased or, as in the case of this project, limited access for research can be granted on application. The database ${ }^{2}$ contains about 260 weather stations located on the island, which includes all stations that were active in the past. About $20 \%$ of these are currently active weather stations. For the selection of the data we had to make compromises, because the stations have data for different time windows, not all records are without gaps, the granted access is limited to data worth of 50000 Euro, and processing and analyzing the data takes time. Hence, the weather stations were first selected to give a good coverage of all regions and all altitudes of the island and then two years long time windows for each station were selected. In order to allow a comparison of the results between different locations, a time window was chosen for which as many stations as possible have data. For the remaining stations, a second time window was chosen for which again as many stations as possible have data, and so on for another 8 time windows. This way we acquired hourly temperature records of several two years long time windows from a total of 59 weather stations. The details of these stations and the time windows are listed in the appendix A.2.

\subsection{ESTIMATION OF SOIL TEMPERATURES}

In order to model the growth of nematode populations we need the temperatures in their natural habitat. The natural habitats of C. elegans include for example rotting fruits, stems, invertebrates, and the upper few centimeters of the soil where oxygen is abundant [122, 123]. The temperatures in these habitats are, to a good approximation, a result of heat diffusion from the surface. Here we try to get viable estimates for the soil temperatures from air temperatures.

For an ideal estimation of the soil temperatures we would need to know the local conditions, e.g. the vegetation, soil color, relief and the thermal diffusivity of the soil, which depends mostly on the type of soil and its composition, the density, and the temporally varying water content. However, for this project it is not important to get an accurate estimate of the temperature series at a specific depth. It is sufficient to get an estimated temperature series with reasonably correct statistical features, in particular the probability distribution and the extreme values, and to know how these features change with depth. On Reunion island is only one weather station that measures air temperature and soil temperatures (Météo-France station 97418110 in Sainte-Marie). We use the data from this station to infer the nec-

$2 \overline{\text { https://publitheque.meteo.fr/ }}$ 
essary parameters to reproduce the soil temperatures by numerically solving the heat equation with the air temperatures as a boundary condition, and use these parameters also for the estimation at other weather stations that do not measure soil temperatures.

The one-dimensional heat equation reads

$$
\frac{\mathrm{d} u}{\mathrm{~d} t}=\vartheta \frac{\mathrm{d}^{2} u}{\mathrm{~d} x^{2}}
$$

where $u=u(x, t)$ is the temperature, $x$ the depth and $\vartheta$ the thermal diffusivity.

The boundary condition at the surface is $u(0, t)=u_{\text {air }}(t)+c$, where $u_{\text {air }}$ is the air temperature and $c$ a constant value to include the difference of air and surface temperature due to the absorption of sunlight and evaporation.

Since we are interested only in the upper few meters of the soil, we can ignore geothermal heat and assume as the lower boundary condition that the temperature at a certain depth $x_{\text {deep }}$ is the average of the surface temperature, $u\left(x_{\text {deep }}, t\right)=\langle u(0, t)\rangle$. In order to choose a reasonable depth $x_{\text {deep }}$ where the temperature is almost constant, we can calculate the amplitude $A(x)$ of a temperature sine wave that travels from the surface to the ground. The upper boundary condition is a sinusoidal temperature $u(0, t)=\langle u(0, t)\rangle+A(0) \sin (\omega t)$ and the lower boundary condition is $u(\infty, t)=\langle u(0, t)\rangle$. The analytical solution of the heat equation with these boundary conditions is $u(x, t)=\langle u(0, t)\rangle+A(x) \sin \left(\omega t-x \sqrt{\frac{\omega}{2 \vartheta}}\right)$, where the amplitude $A(x)=A(0) \exp \left(-x \sqrt{\frac{\omega}{2 \vartheta}}\right)$ decreases exponentially with the depth [124]. For a typical annual temperature variation between $20^{\circ} \mathrm{C}$ and $30^{\circ} \mathrm{C}$ over the year $\left(A(0)=5^{\circ} \mathrm{C}\right)$, and a thermal diffusivity of $\vartheta=0.6 \times 10^{-6} \mathrm{~m}^{2} / \mathrm{s}$, the amplitude decreases to $A(x)<0.1^{\circ} \mathrm{C}$ if $x>9.58 \mathrm{~m}$. Since the thermal diffusivity used for this calculation is a relatively large value for soil [125], $x>9.58 \mathrm{~m}$ can be considered an upper bound for the depth where the amplitude is smaller than $0.1^{\circ} \mathrm{C}$. Hence, for the numerical solution of the heat equation we assume as a lower boundary condition that the temperature is constant, $u\left(x_{\text {deep }}, t\right)=\langle u(0, t)\rangle$, at depth $x_{\text {deep }}=10 \mathrm{~m}$.

Next, we compare the numerical solution of the heat equation with soil temperature records from a weather station on Reunion island to infer the temperature shift $c$ and the thermal diffusivity $\vartheta$. The available soil temperatures were recorded at depths of $20 \mathrm{~cm}, 50 \mathrm{~cm}$ and $100 \mathrm{~cm}$. These soil temperature records can be reproduced to a satisfying degree by solving the heat equation as described above using the temperature shift $c=2 \mathrm{~cm}$ and a thermal diffusivity of $\vartheta=0.25 \times 10^{-6} \mathrm{~m}^{2} / \mathrm{s}$. The value for the thermal diffusivity resembles typical values for soils of dry sand or wet clay [125]. Figure 6.11 shows a comparison of the measured and estimated soil temperatures using these parameters. While the measured and estimated tempera- 
tures are quite close in times without precipitation (e.g. figure 6.11A), the larger time windows (figure 6.11B and figure 6.11C) show larger deviations due to sudden temperature drops in the measured soil temperatures. These temperature drops coincide with high precipitation.

Figure 6.12 shows a comparison of the probability distributions of measured and estimated temperatures. In all three cases the measured data show higher probabilities for low temperatures compared to the estimated data, which is likely due to the effect of precipitation. More importantly, the overall shape of the distribution and the shrinking width of the distribution with depth seems to be sufficiently well reproduced.

\subsection{RESULTS}

Theoretically, the model described in section 6.4 could be used to simulate different species and strains of nematodes. However, sufficient data is available only for C. elegans strain N2. In order to simulate strains that are adapted to higher or lower temperatures than N2, we can create virtual strains by assuming that they behave exactly the same as N2 except that they experience shifted temperatures. For example, $\mathrm{N} 2$ has a maximum growth rate at the constant temperature $T_{\mathrm{id}}=24^{\circ} \mathrm{C}$ (see figure 6.9B). To simulate a virtual strain with $T_{\text {id }}=23^{\circ} \mathrm{C}$ we shift all temperature by $1{ }^{\circ} \mathrm{C}$.

With this method we can simulate the population and measure the growth rates for different phenotypes by varying the ideal temperature $T_{\mathrm{id}}$ and find the best adapted ideal temperature $T_{\mathrm{id}}^{*}$ for a specific temperature record. The temperature records we use are the estimated soil temperatures (as described in section 6.6) from the acquired air temperature records (see section 6.5).

As a first example, figure 6.13 shows the growth rate depending on the ideal temperature for one of these temperature scenarios. From the growth rate curve we can measure the deviation $\delta=T_{\mathrm{id}}^{*}-\langle T\rangle$, where $\langle T\rangle$ is the average soil temperature.

The deviations for all available weather stations and with soil temperatures estimated at different depths are shown in figure 6.14 (for specific values see appendix A.2).

In contrast to the deviations in the theoretical examples that are positive or negative (see section 6.3), all predicted deviations for the nematodes are positive. This difference is a consequence from the different shapes of the fitness functions. In the theoretical examples we used symmetric Gaussian fitness functions, but the fitness function of the nematodes is strongly asymmetric (see figure 6.9B), with a long increase at low temperatures followed by a sudden decline shortly above the temperature of the fitness maximum. Therefore a temperature series with a mean temperature above the ideal temperature 
A depth: $20 \mathrm{~cm}$

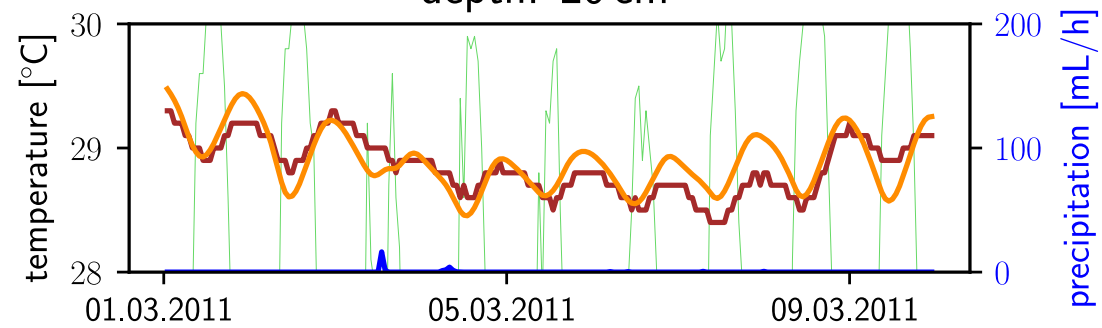

B depth: $50 \mathrm{~cm}$

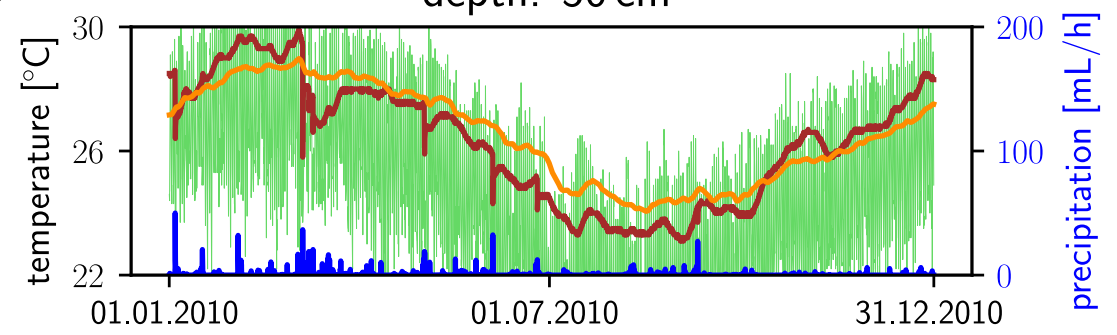

C depth: $100 \mathrm{~cm}$

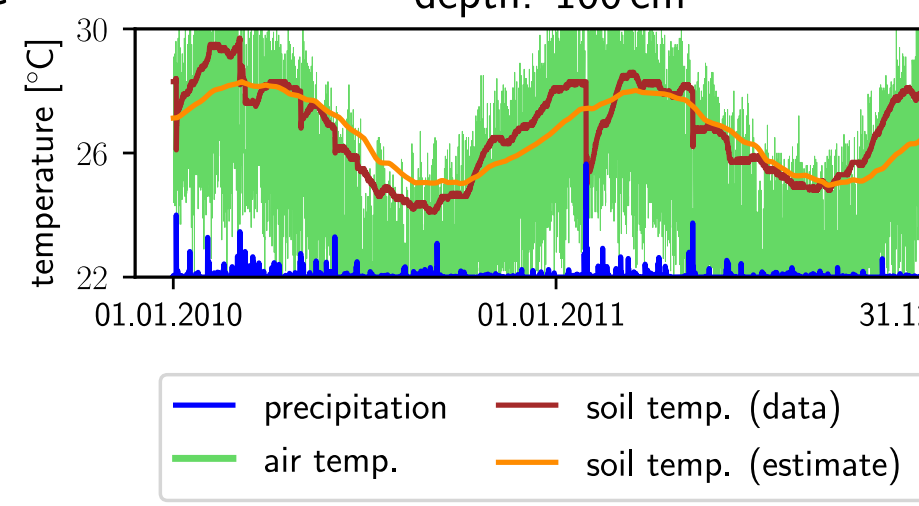

Figure 6.11: Comparison of measured and estimated soil temperatures. The plot shows the soil temperature data and the soil temperatures that were estimated from the air temperature as described in the main text (at the three different depths where soil temperature data is available). Independent of the depth, each plot also shows the raw data of the air temperature and the precipitation. 

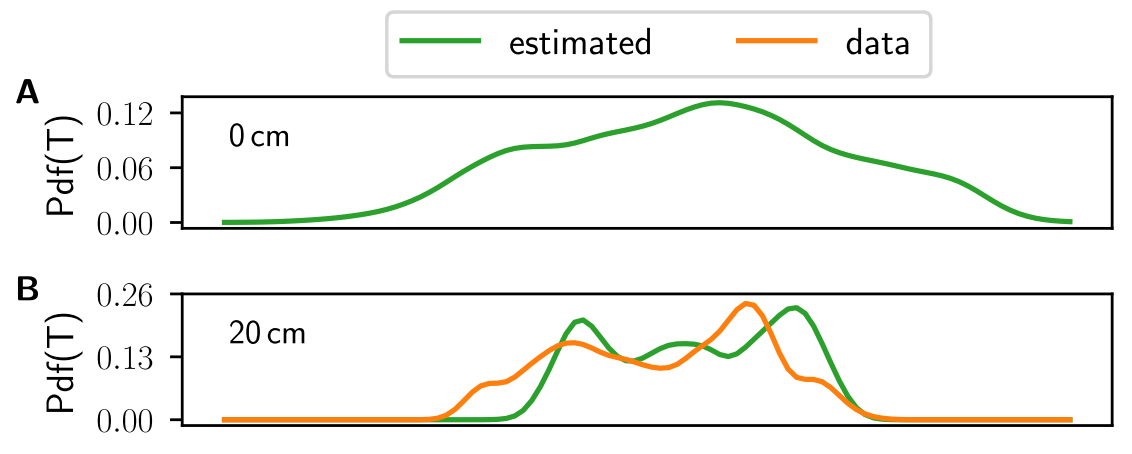

C
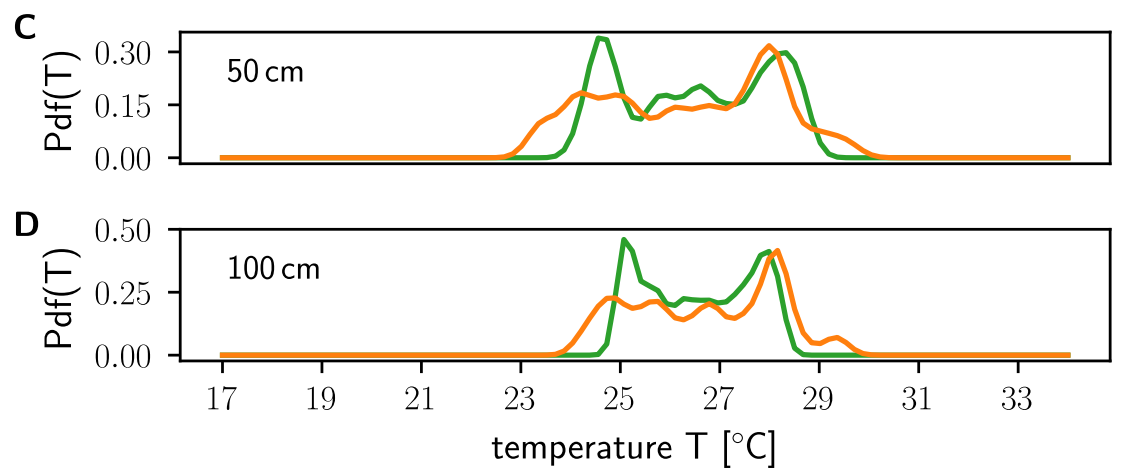

Figure 6.12: Probability densities of measured and estimated soil temperatures. (A) shows the probability density of the surface temperature $\left(u(0)=u_{\text {air }}+c\right)$, which is used as the upper boundary condition for the solution of the heat equation. (B), (C) and (D) show the probability density of the measured soil temperatures and the estimated soil temperatures. All curves are results of a kernel-density estimation using Gaussian kernels. 
would result in a small average fitness, unless the temperature range is unusually small.

From the surface to $5 \mathrm{~cm}$ depth the deviations increase approximately by one degree. The reason for this increase is that the temperature range that allows survival has to include the whole range of environmental temperatures. Since the range of environmental temperatures shrinks with the depth (see figure 6.12), the range of temperatures in which the ideal temperature $T_{\mathrm{id}}$ can be optimized increases with the depth.

From $10 \mathrm{~cm}$ to $20 \mathrm{~cm}$ the deviations slowly decrease, because the distributions of all temperature series approach the average temperature at high depth, hence the deviations have to approach zero deep in the soil.

Since the deviations do not change much from $5 \mathrm{~cm}$ to $20 \mathrm{~cm}$ and since nematodes can avoid unfavorable temperatures by moving deeper in the soil [122], we will use the deviations at $10 \mathrm{~cm}$ as representative results in the next steps.

Two years long temperature records may give a biased view on the local climate. The check whether the results change over time, figure 6.15A shows the deviations for different years. Most temperature records start at 2010, but the few other records do not indicate that the results are very sensitive to the year.

Further, it would be interesting to know whether the deviations depend on the location of the weather station. Figure 6.15B shows that the deviations tend to increase with the altitude of the weather station. A similar trend can be observed on the map shown in figure 6.16, where the weather stations with the small deviations are mostly located along the coast and weather stations with large deviations are more often on the mountains in the central part of the island. 


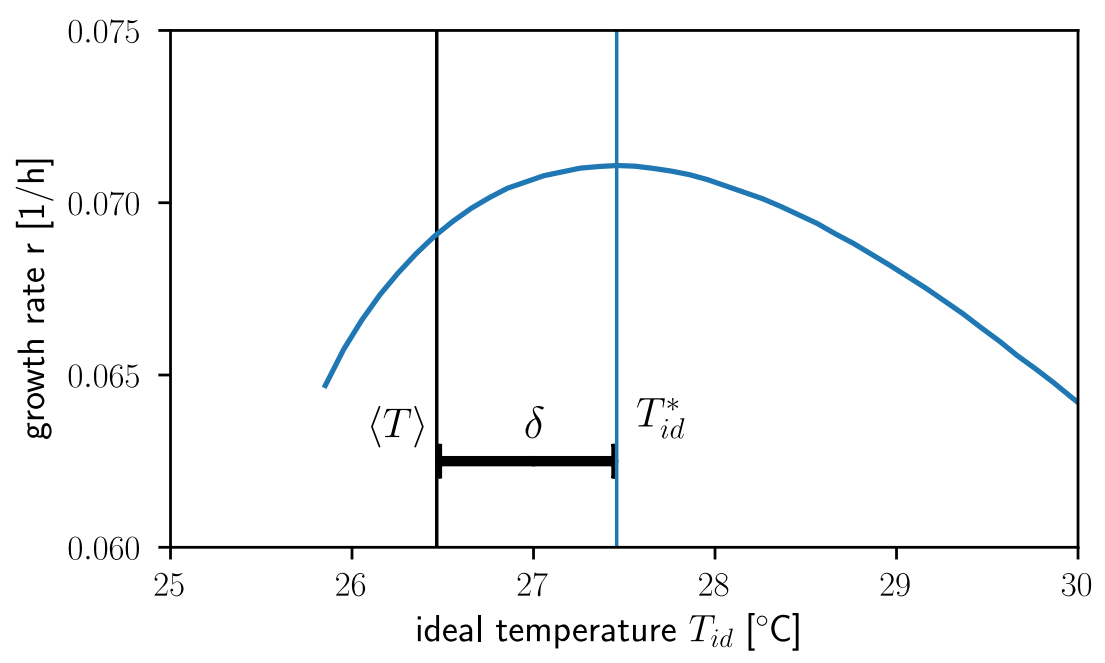

Figure 6.13: Example to illustrate the analysis. The blue curve shows the growth rates $r$ predicted by the nematodes growth model assuming a shifted ideal temperature $T_{\text {id }}$ to simulate adaptation and using a two years long temperature series from the weather station in Sainte-Marie on Reunion island. The vertical blue line marks the best ideal temperature $T_{\mathrm{id}}^{*}$, where the growth rate is at the maximum. The vertical black line marks the timeaverage of the temperature $\langle T\rangle$. The deviation between the two is $\delta=T_{\mathrm{id}}^{*}-\langle T\rangle$.

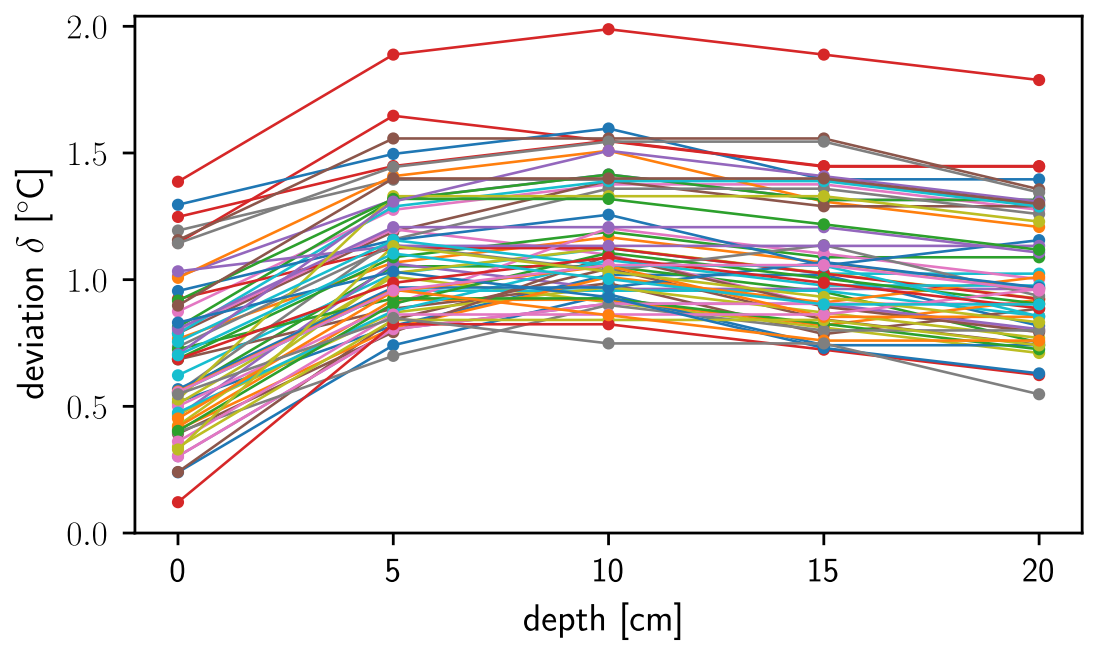

Figure 6.14: Predicted deviations depending on depth. The predictions are based on the estimated soil temperatures. The lines connect the values that belong to the same weather station. 

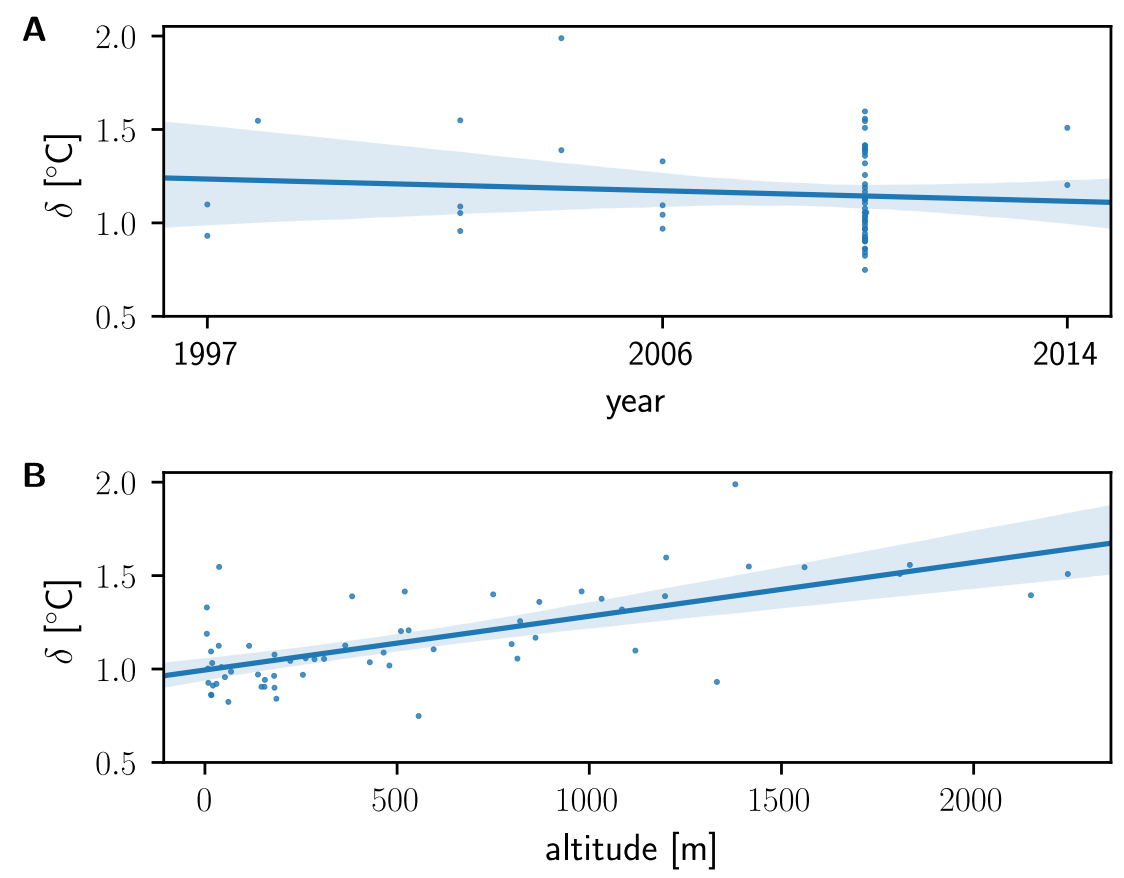

Figure 6.15: Dependence of the predicted deviation on altitude and year. Shown are the predicted deviations $\delta$ versus the starting date of the two years long temperature record (A) and the altitude of the weather station (B). All deviation values based on soil temperature estimates at $10 \mathrm{~cm}$. Lines are linear regressions with the shaded area showing the $95 \%$ confidence interval of the slope. 

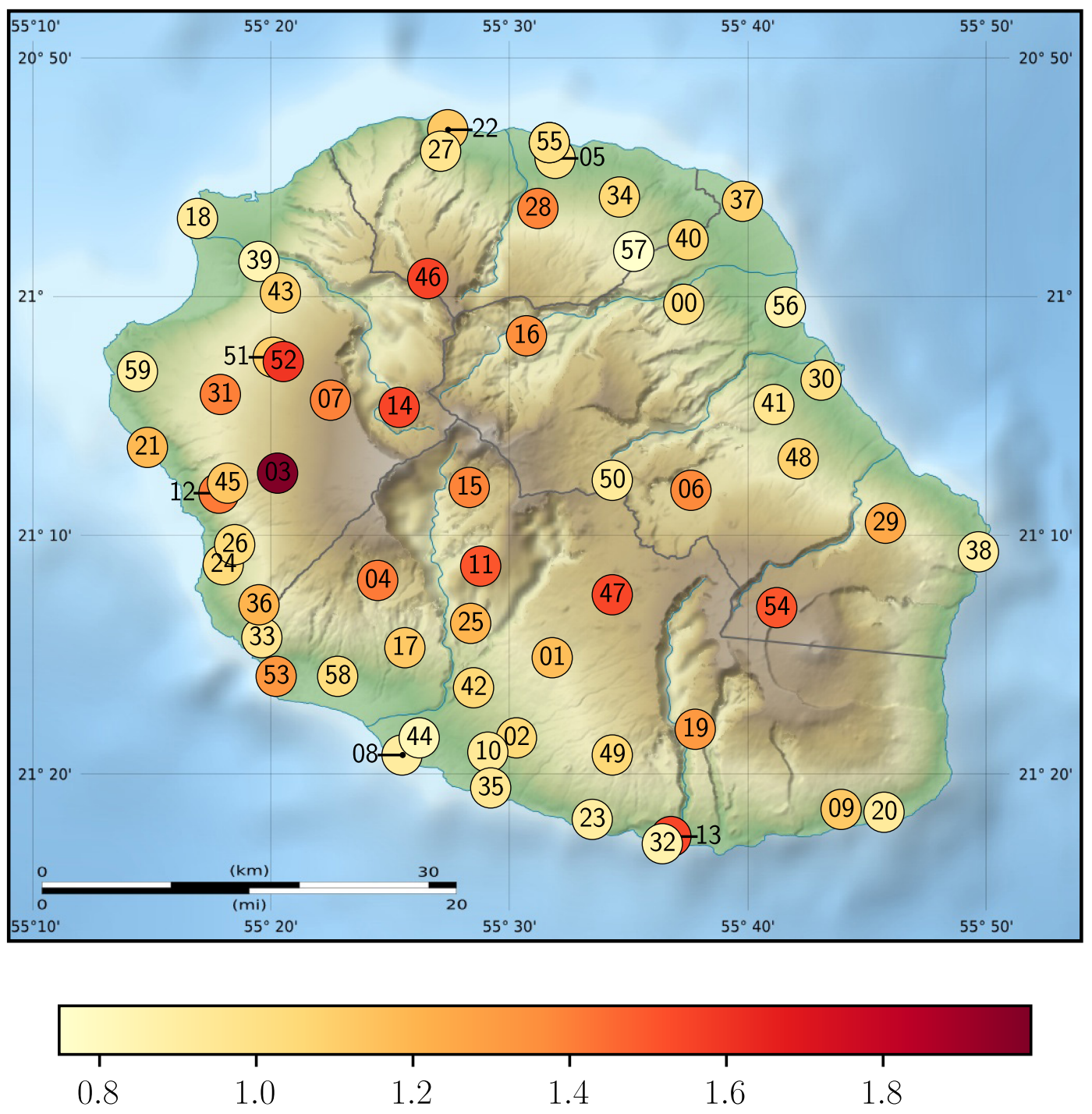

Figure 6.16: Geographic map of the weather stations color-coded by the deviation. The colors show the deviations $\delta$ calculated at $10 \mathrm{~cm}$. The numbers are labels assigned to the weather stations in arbitrary order to identify the stations in the lists in appendix A.2. Background image: Eric Gaba, "La Réunion department relief location map", CC-BY-SA 3.o. 
The ultimate goal of this project is to compare the predicted deviations with deviations measured with nematode strains from different locations on Reunion island. If the measurements confirm the predictions, we know that nematodes do not simply adapt to the average environmental temperature in their natural habitat, but adapt to a different temperature that is optimal for the specific temperature fluctuation in their environment. In addition, this would be an example of a general effect for adaptation to varying environments, that is likely relevant for many other species in changing environments as well. However, the experiments to measure the deviations with nematodes take time and it is possible that the comparison of predictions with only a few real strains is inconclusive because the presented method of prediction has some sources of errors. One problem is that the original locations of the nematode strains that are available from Reunion island are not exactly the same as the locations of the weather stations and in mountainous regions like Reunion island the temperatures at a few hundred meter distance can be very different. Secondly, we assume that the growth rate curves of strains which are adapted to different temperatures have the same shape and are just shifted to higher or lower temperatures. Third, the estimation of soil temperatures is certainly not precise. Since the results between $5 \mathrm{~cm}$ and $20 \mathrm{~cm}$ do not change much, assuming a wrong thermal diffusivity would not have a substantial impact. But ignoring the effect of precipitation and assuming a wrong shift between air and surface temperature cause systematic errors in the calculations of the deviations. Fourth, the yearly average temperatures on Reunion island increases [126], which causes a systematic error if the time window of the weather data is not the same as the time frame to which the nematode strains are adapted.

An ideal setup would be to directly measure soil temperatures for several years at the same locations where the nematodes were collected. The results so far are a proof-of-concept, because the predicted range of deviations is between $0.5^{\circ} \mathrm{C}$ and $1.5^{\circ} \mathrm{C}$, which should be strong enough to detect in experiments with nematodes. 



\section{CONCLUSION}

\subsection{SUMMARY AND DISCUSSION}

\subsubsection{Overview}

Almost all real species evolve in changing environments. How do environmental fluctuations affect the evolutionary dynamics of species? In this thesis, we addressed this question in two ways.

First, we studied the implications of changing payoff values in evolutionary game theory (chapter 3 ) and their connection to evolutionary games in structured populations (chapter 4). In chapter 3 we learned that averaged payoff values are generally not suited as a simplified representation of changing payoff values, because the resulting evolutionary dynamics can be qualitatively different. Building on this, we learned in chapter 4 that stochastically fluctuating payoff values might serve as a simplified representation of evolutionary games on networks and in spatially distributed populations, because the payoff in structured populations is subject to inherent noise due to the fact that every individual interacts with a different subset of other individuals.

Second, we developed two models for evolutionary processes of microbiological species in which the effects of changing environments can be tested experimentally (chapter 5 and chapter 6). To summarize, our model for the evolution of shared antibiotic resistance in bacteria allows new insights in the evolutionary dynamics, the invadability, and the mutational stability of shared antibiotic resistance, but predictions for fluctuating antibiotic concentrations require experimental measurements of the fitness functions. Our model for the adaptation of nematodes to changing temperatures predicts that the constant temperature at which the nematodes have the maximum growth rate is typically about $1^{\circ} \mathrm{C}$ higher than the average temperature of the changing temperatures in their natural habitats. With ongoing experiments we aim to test these predictions. 


\subsubsection{Chapter 3: Evolutionary game theory with payoff fluctuations}

In fluctuating environments it is unlikely that the payoff values of evolutionary games are constant. In chapter 3 we studied the more realistic case of time-dependent payoff values and showed that the resulting dynamics is qualitatively different to the dynamics resulting from the averaged payoff values.

Depending on the type, the intensity, and the correlations of the payoff fluctuations, the stationary states can be shifted or change their stability and stationary states can emerge or disappear.

In evolutionary game theory with constant payoff values, two species that coexist in an evolutionarily stable state necessarily receive equal payoffs. We showed that with fluctuating payoff values, the timeaveraged payoff that two coexisting species receive is generally not equal.

Evolutionary games with constant payoff values are traditionally classified regarding the order of the values in their payoff matrix. Since this method is limited to constant payoff values, we developed a new classification that is based on the evolutionary dynamics instead of the payoff matrix. Using this method we can understand the change of the evolutionary dynamics due to payoff fluctuations as transitions from one game to another game. An interesting example is the transition from a Prisoner's Dilemma to a Hawk-Dove game. Since decades, a central question in evolutionary game theory is how cooperation can evolve despite the theoretical result that cooperators should be outperformed by defectors in an evolutionary Prisoner's Dilemma. With the transition from a Prisoner's Dilemma to a Hawk-Dove game caused by payoff fluctuations we found a new mechanism that can enable the evolution of cooperation.

The results of this chapter can be useful for understanding evolutionary games of real species in experiments.

The traditional terminology for evolutionary games is based on linear fitness functions $M \mathbf{x}$, where $M$ is the payoff matrix which defines the type of the game, e.g. Prisoner's Dilemma, Hawk-Dove game, etc. However, all experiments on evolutionary games with microbes show that realistic fitness functions are typically nonlinear, hence the traditional terminology can not be applied. Our new method of classification contains the classic games of linear fitness functions as special cases and in addition can also be applied to evolutionary games with nonlinear fitness functions.

Further, experiments on evolution of microbes which were transferred from their natural environment to the controlled conditions in a lab need to take into account that the evolutionary dynamics may be different and consider to imitate the fluctuations of the natural environment in order to study their evolution in the wild. 


\subsubsection{Chapter 4: Evolutionary games on networks}

It is well known that the dynamics of evolutionary games on networks and in spatially distributed populations is different to evolutionary games in well-mixed populations. In chapter 4 we showed that this difference is similar to the difference between evolutionary games with and without payoff fluctuations. The reason for this similarity is that the payoff in a network game is subject to noise that is inherent to the network, because every node interacts with a different subset of other nodes.

Evolutionary games in unstructured and in structured populations share many concepts, but are studied with different methods. The introduction of fluctuating payoffs in unstructured populations and the interpretation of structured populations as inherent payoff noise, could result in a common method and comparable results.

We demonstrated the similarity only in four evolutionary games on random networks. It would be interesting to study more systematically whether results from evolutionary games in structured populations can be reproduced in evolutionary games in unstructured populations with payoff fluctuations. This may help to understand the effects of different network types on the evolutionary dynamics because it reduces a network to a stochastic process.

\subsubsection{Chapter 5: Shared antibiotic resistance}

Experiments demonstrated that bacteria can inactivate an antibiotic and thereby protect other bacteria. This is an example of a real evolutionary game, but the classical models of evolutionary game theory can not be applied to this example because they are based on abstract parameters like payoff values and on linear fitness functions. In order to bridge this gap we developed a model for the evolution of shared antibiotic resistance based on measurable parameters such that the predictions can be tested in experiments.

At this time, only predictions for constant antibiotic concentrations are possible, because predictions for variable antibiotic concentrations require to know the exact fitness functions of the bacteria strains. Therefore, the results remain preliminary in the context of this thesis, but apart from the aim to study the effect of fluctuating environments the results are interesting on their own because they allow new insights in the evolution of antibiotic resistance.

First of all, common one-dimensional measures of resistance like the minimum inhibitory concentration are insufficient to understand shared antibiotic resistance. We showed that we need to consider resistance as a two-dimensional property of bacteria to understand this phenomenon.

Second, our model reproduces a number of phenomena that are known 
from experiments, including the inoculum effect, the observation that the evolution of resistance is usually faster than the loss of resistance, that evolution of resistance is most likely for intermediate concentrations of the antibiotic, and that resistant and sensitive strains can coexist in an evolutionary stable state.

Third, the model shows that shared antibiotic resistance, although evolutionarily stable (regarding perturbations of the population sizes), is not mutationally stable (regarding perturbations of the phenotypes). Surprisingly, a resistant and a sensitive strain which coexist due to shared antibiotic resistance and therefore are together resistant to the antibiotic, can be invaded and completely replaced by a third strain that is sensitive to the antibiotic.

Although we developed the model to understand shared antibiotic resistance, it could be extended in the future to include other phenomena which are related to antibiotic resistance, for example antibiotic-producing bacteria strains, biofilms, or more than one type of antibiotic, which leads to synergistic or antagonistic effects and the evolution of cross-resistance and cross-sensitivity.

\subsubsection{Chapter 6: Adaptation to fluctuating temperatures}

In chapter 6 we studied how a species adapts to changing environments. We showed that the environmental fluctuations result in a deviation between the ideal environmental condition, in which the species has the highest fitness, and the average of the environmental conditions to which the species is adapted.

An ideal species for an experimental test of this effect would be a microbial species for which strains are available which have adapted to different changing conditions, and for which the general sensitivity to these conditions is already well known. Nematodes qualify for this attempt because their temperature sensitivity is an active field of research and strains that adapted in different local climates are available. Based on existing data on the temperature sensitivity of the development and reproduction of $C$. elegans, we developed a model for the growth of nematode populations. We tested the model by comparing predicted growth rates to existing results from the literature for constant temperatures and to new experimental results for switching temperatures. Using this model, we predicted the deviations between the ideal temperature and the average environmental temperature based on temperature records from weather stations of Reunion island. The predicted deviations are between $0.5^{\circ} \mathrm{C}$ and $1.5^{\circ} \mathrm{C}$, with the tendency of lower values at weather stations near the coast and higher values in the central part of the island at high altitudes.

The next step will be the comparison of the predicted deviations with measurements from ongoing experiments conducted at the Biotech- 
nology Center (TU Dresden) using nematode strains from different locations on Reunion island.

The temperature adaptation of nematodes is just one example of a general effect in the adaptation to changing conditions. Theoretically, many other species and other fluctuating environmental parameters should show similar deviations between ideal and average conditions. Consequently, when evaluating the impact of changing environments such as global warming or ocean acidification on ecosystems, it is not sufficient to consider only the change of the average values.

\subsection{OUTLOOK: TOWARDS APPLIED EVOLUTIONARY DYNAMICS}

During the history of evolutionary dynamics it was widely accepted that evolution is a slow process. "We see nothing of these slow changes in progress, until the hand of time has marked the long lapse of ages, and then so imperfect is our view into long past geological ages, that we only see that the forms of life are now different from what they formerly were." (Charles Darwin, On the Origin

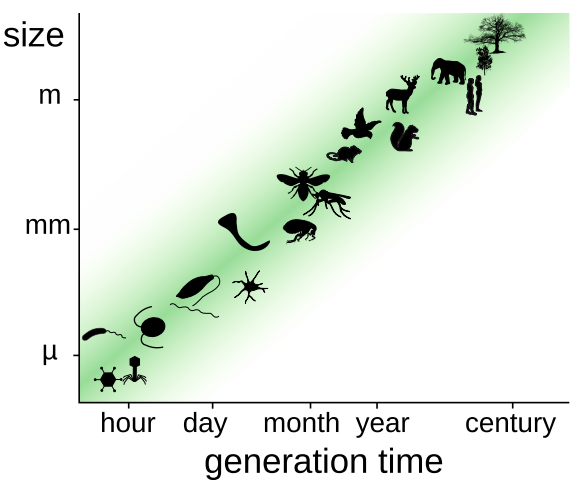

Figure 7.1: Time scales of evolution. of Species, 1859). As a consequence of this hypothesis, the aim of evolutionary dynamics in the past was mainly to understand how species have evolved.

Today, we know from many experiments in microbiology that evolution can be fast enough that we can observe it and even perform experiments. For example, bacteria can increase their antibiotic resistance by a factor of 3000 in just a few days [127]. This naturally raises the question whether we can also control and manipulate the evolutionary process.

In antibiotic resistance management it is already common practice to consider the evolutionary dynamics of bacteria. Strategies like reducing the number of unnecessary antibiotic treatments, avoiding too early discontinuation of treatments, and the use of drug cycling in hospitals have the goal to prevent the evolution of resistance. But these are just the obvious applications of evolutionary dynamics to avoid antibiotic resistance of bacteria. Taking into account the interactions and the coevolution of bacteria strains could show more effective methods. In addition, there are many other interesting and relevant examples of microbial evolution where evolutionary dynamics could be applied to manipulate the evolutionary process in a desired way, for example the coevolution of pathogens and antibodies [128], 
virus strains [129], cancer cells [130], or the human microbiome [131]. And the list of equally relevant but unknown cases of microbial evolution is probably much longer.

As noted in the introduction of this thesis, understanding evolution can be challenging because evolving species constitute a complex, nonlinear, high dimensional, heterogeneous, stochastic system. But for the same reason, there is a high chance that small changes of the conditions can lead to different and unexpected results. With better models for the evolution of specific microbial systems that can predict how changing a condition affects the evolutionary dynamics, more sophisticated applications of evolutionary dynamics could be developed. 


\section{APPENDIX}

\section{A.I PARAMETERS FOR FIGURE $5 \cdot 4$}

Table A.I contains all parameters to reproduce figure 5.4. In the following we give further explanation of the meaning and choice of the parameters.

If the environmental concentration $c_{\text {env }}$ is too large all bacteria die and if it is too low no bacteria strain suffers from the antibiotic. We choose the value to be in the range where coexistence is possible. The boundaries of this range depend on the present phenotypes and the permeability of the surface between the environment and the local habitat.

The extracellular concentration $c_{\mathrm{ec}}$ is an equilibrium of the total influx and the total degradation of antibiotics. Since the influx depends on the surface $A_{s}$ through which the antibiotics diffuses from the environment to the local habitat, and the degradation depends on the population size $N$, the equilibrium depends on the ratio of $A_{s}$ and $N$. For simplicity, we assume this ratio to be constant. A constant ratio could result from a constant population size $N$, e.g. because of limited availability of space and nutrition. Another possible scenario with a constant ratio is a bacteria culture that lives on a surface, as illustrated in figure 5.2A, since the area $A_{s}$ of the covered surface scales linearly with the number of cells $N$. The latter interpretation is useful to get a simple estimate. If we assume the surface is densely covered by bacteria of size $1 \mu \mathrm{m}$ and we ignore boundary effects the ratio is $\frac{A_{s}}{N}=1 \mu \mathrm{m}^{2} /$ cell.

The permeability $P_{\text {loc }}$ is the diffusion coefficient of the surface between the environment and the local habitat multiplied with the ratio $\frac{A_{s}}{N}$, such that $P_{\text {loc }}\left(c_{\mathrm{env}}-c_{\mathrm{ec}}\right)$ is the antibiotic influx the culture has to deal with per cell. As described above, the value is chosen to be in the range where coexistence is possible.

In figure $5.4 \mathrm{~A}$ and $5.4 \mathrm{~B}$ we assume the presence of a phenotype A. As the maximum degradation rate for this phenotype we use $1 \times 10^{6} \frac{\text { molecules }}{\text { cell-second }}$, which has been estimated for E. coli bacteria which are ampicillin-resistant due to a $\beta$-lactamase gene [89].

We chose the resistance of phenotype A to be $\mathrm{MIC}_{\mathrm{A}}=500 \mu \mathrm{g} / \mathrm{mL}$ and its maximum degradation rate $I_{\max , \mathrm{A}}=1 \times 10^{6} \frac{\text { molecules }}{\text { cell. second }}$. This 


\begin{tabular}{c|c|c|c} 
Figure & Description & Variable & Value \\
\hline \hline A,B \& C & $\begin{array}{c}\text { Environmental } \\
\text { antibiotic concentration }\end{array}$ & $c_{\mathrm{env}}$ & $5000 \mu \mathrm{g} / \mathrm{mL}$ \\
\hline A,B \& C & $\begin{array}{c}\text { Surface area, through which } \\
\text { the antibiotic diffuses } \\
\text { from the environment and the } \\
\text { local habitat, per bacteria cell }\end{array}$ & $\frac{A_{s}}{N}$ & $1 \mu \mathrm{m}^{2} / \mathrm{cell}$ \\
\hline A,B \& C & $\begin{array}{c}\text { Permeability of the surface } \\
\text { between the environment } \\
\text { and the local habitat }\end{array}$ & $P_{\mathrm{loc}}$ & $0.05 \mu \mathrm{m}^{4} / \mathrm{s} \cdot$ cell \\
\hline B \& C & $\begin{array}{c}\text { Maximum degradation rate } \\
\text { of a cell of phenotype A }\end{array}$ & $I_{\max , \mathrm{A}}$ & $\approx 5.8 \times 10^{-10} \frac{\mathrm{molecules}}{\mathrm{cell} \cdot \mathrm{second}}$ \\
\hline B \& C & $\begin{array}{c}\text { Rell.second } \\
\text { ofive cell wall permeability } \\
\text { of cells of phenotype A }\end{array}$ & $\frac{P_{\mathrm{c}, \mathrm{A}}}{P_{\mathrm{loc}}}$ & 2.3248 \\
\hline C & $\begin{array}{c}\text { Maximum degradation rate } \\
\text { of a cell of phenotype B }\end{array}$ & $I_{\mathrm{max}, \mathrm{B}}$ & $0.7 \times 10^{-10} \frac{\mathrm{\mu g}}{\text { cell. second }}$ \\
\hline C & $\begin{array}{c}\text { Relative cell wall permeability } \\
\text { of cells of phenotype B }\end{array}$ & $\frac{P_{\mathrm{c}, \mathrm{B}}}{P_{\mathrm{loc}}}$ & 0.7 \\
\hline
\end{tabular}

Table A.1: Parameters for figure 5.4. The first three rows define the environment, which is the same in all three figures. The following two rows define the phenotype $\mathrm{A}$, which is the same in figure $5.4 \mathrm{~B}$ and $5.4 \mathrm{C}$. The last two rows define phenotype B, which is required only in figure $5.4 \mathrm{C}$.

determines the permeability of phenotype A. The phenotype of B is chosen such that A and B coexist and their population share is close to $50 \%$. 
A.2 Metadata AND Results of WeAther DAta ANAlysis

\begin{tabular}{|c|c|c|c|c|}
\hline Label & ID & Coordinates & Altitude [m] & Years \\
\hline oO & 97402240 & $55.622 \mathrm{E} ; 21.005 \mathrm{~S}$ & 480 & 2010 and 2011 \\
\hline O1 & 97422465 & $55.53 \mathrm{E} ; 21.252 \mathrm{~S}$ & 860 & 2010 and 2011 \\
\hline 02 & 97416455 & $55.505 \mathrm{E} ; 21.308 \mathrm{~S}$ & 285 & 2002 and 2003 \\
\hline 03 & 97413512 & $55.338 \mathrm{E} ; 21.123 \mathrm{~S}$ & 1380 & 2004 and 2005 \\
\hline 04 & 97414409 & $55.408 \mathrm{E} ; 21.198 \mathrm{~S}$ & 980 & 2010 and 2011 \\
\hline $\mathrm{O} 5$ & 97418123 & $55.532 \mathrm{E} ; 20.903 \mathrm{~S}$ & 68 & 2010 and 2011 \\
\hline o6 & 97406220 & $55.627 \mathrm{E} ; 21.135 \mathrm{~S}$ & 1032 & 2010 and 2011 \\
\hline 07 & 97415566 & $55.375 \mathrm{E} ; 21.072 \mathrm{~S}$ & 2149 & 2010 and 2011 \\
\hline o8 & 97416463 & $55.425 \mathrm{E} ; 21.32 \mathrm{~S}$ & 21 & 2010 and 2011 \\
\hline 09 & 97417360 & $55.732 \mathrm{E} ; 21.358 \mathrm{~S}$ & 115 & 2010 and 2011 \\
\hline 10 & 97416465 & $55.485 \mathrm{E} ; 21.318 \mathrm{~S}$ & 156 & 2010 and 2011 \\
\hline 11 & 97403410 & $55.48 \mathrm{E} ; 21.188 \mathrm{~S}$ & 1808 & 2014 and 2015 \\
\hline 12 & 97413524 & $55.297 \mathrm{E} ; 21.137 \mathrm{~S}$ & 520 & 2010 and 2011 \\
\hline 13 & 97412376 & $55.613 \mathrm{E} ; 21.377 \mathrm{~S}$ & 37 & 1998 and 1999 \\
\hline 14 & 97408580 & $55.423 \mathrm{E} ; 21.077 \mathrm{~S}$ & 1415 & 2002 and 2003 \\
\hline 15 & 97424410 & $55.472 \mathrm{E} ; 21.133 \mathrm{~S}$ & 1197 & 2010 and 2011 \\
\hline 16 & 97421210 & $55.512 \mathrm{E} ; 21.027 \mathrm{~S}$ & 870 & 2010 and 2011 \\
\hline 17 & 97414431 & $55.427 \mathrm{E} ; 21.245 \mathrm{~S}$ & 365 & 2010 and 2011 \\
\hline 18 & 97407520 & $55.282 \mathrm{E} ; 20.945 \mathrm{~S}$ & 9 & 2010 and 2011 \\
\hline 19 & 97412336 & $55.63 \mathrm{E} ; 21.302 \mathrm{~S}$ & 1085 & 2010 and 2011 \\
\hline 20 & 97417380 & $55.762 \mathrm{E} ; 21.36 \mathrm{~S}$ & 30 & 2010 and 2011 \\
\hline 21 & 97415590 & $55.247 \mathrm{E} ; 21.105 \mathrm{~S}$ & 5 & 2010 and 2011 \\
\hline 22 & 97411111 & $55.457 \mathrm{E} ; 20.883 \mathrm{~S}$ & 36 & 2010 and 2011 \\
\hline 23 & 97405480 & $55.558 \mathrm{E} ; 21.365 \mathrm{~S}$ & 155 & 2010 and 2011 \\
\hline 24 & 97413545 & $55.3 \mathrm{E} ; 21.187 \mathrm{~S}$ & 222 & 2006 and 2007 \\
\hline 25 & 97403435 & $55.473 \mathrm{E} ; 21.228 \mathrm{~S}$ & 510 & 2014 and 2015 \\
\hline 26 & 97413550 & $55.308 \mathrm{E} ; 21.173 \mathrm{~S}$ & 429 & 2010 and 2011 \\
\hline 27 & 97411126 & $55.452 \mathrm{E} ; 20.898 \mathrm{~S}$ & 138 & 2010 and 2011 \\
\hline 28 & 97418167 & $55.52 \mathrm{E} ; 20.938 \mathrm{~S}$ & 383 & 2004 and 2005 \\
\hline 29 & 97419320 & $55.763 \mathrm{E} ; 21.158 \mathrm{~S}$ & 820 & 2010 and 2011 \\
\hline 30 & 97410238 & $55.718 \mathrm{E} ; 21.058 \mathrm{~S}$ & 43 & 2010 and 2011 \\
\hline 31 & 97415541 & $55.298 \mathrm{E} ; 21.068 \mathrm{~S}$ & 750 & 2010 and 2011 \\
\hline 32 & 97412384 & $55.607 \mathrm{E} ; 21.382 \mathrm{~S}$ & 17 & 2010 and 2011 \\
\hline
\end{tabular}




\begin{tabular}{|c|c|c|c|c|}
\hline Label & ID & Coordinates & Altitude [m] & Years \\
\hline 33 & 97401540 & $55.327 \mathrm{E} ; 21.238 \mathrm{~S}$ & 180 & 2010 and 2011 \\
\hline 34 & 97420150 & $55.577 \mathrm{E} ; 20.93 \mathrm{~S}$ & 262 & 2010 and 2011 \\
\hline 35 & 97416480 & $55.487 \mathrm{E} ; 21.343 \mathrm{~S}$ & 52 & 2002 and 2003 \\
\hline 36 & 97413580 & $55.325 \mathrm{E} ; 21.215 \mathrm{~S}$ & 530 & 2010 and 2011 \\
\hline 37 & 97409230 & $55.663 \mathrm{E} ; 20.933 \mathrm{~S}$ & 16 & 2006 and 2007 \\
\hline 38 & 97419350 & $55.828 \mathrm{E} ; 21.178 \mathrm{~S}$ & 181 & 2010 and 2011 \\
\hline 39 & 97415511 & $55.325 \mathrm{E} ; 20.975 \mathrm{~S}$ & 186 & 2010 and 2011 \\
\hline 40 & 97409240 & $55.625 \mathrm{E} ; 20.96 \mathrm{~S}$ & 181 & 2010 and 2011 \\
\hline 41 & 97410265 & $55.685 \mathrm{E} ; 21.075 \mathrm{~S}$ & 255 & 2006 and 2007 \\
\hline 42 & 97416410 & $55.475 \mathrm{E} ; 21.273 \mathrm{~S}$ & 310 & 2010 and 2011 \\
\hline 43 & 97415516 & $55.34 \mathrm{E} ; 20.997 \mathrm{~S}$ & 595 & 2010 and 2011 \\
\hline 44 & 97416415 & $55.437 \mathrm{E} ; 21.308 \mathrm{~S}$ & 61 & 2010 and 2011 \\
\hline 45 & 97413520 & $55.303 \mathrm{E} ; 21.13 \mathrm{~S}$ & 798 & 2010 and 2011 \\
\hline 46 & 97411170 & $55.443 \mathrm{E} ; 20.987 \mathrm{~S}$ & 1834 & 2010 and 2011 \\
\hline 47 & 97422440 & $55.572 \mathrm{E} ; 21.208 \mathrm{~S}$ & 1560 & 2010 and 2011 \\
\hline 48 & 97410280 & $55.702 \mathrm{E} ; 21.113 \mathrm{~S}$ & 465 & 2002 and 2003 \\
\hline 49 & 97405420 & $55.572 \mathrm{E} ; 21.32 \mathrm{~S}$ & 813 & 2010 and 2011 \\
\hline 50 & 97410286 & $55.572 \mathrm{E} ; 21.128 \mathrm{~S}$ & 1332 & 1997 and 1998 \\
\hline 51 & 97415535 & $55.335 \mathrm{E} ; 21.042 \mathrm{~S}$ & 1120 & 1997 and 1998 \\
\hline 52 & 97415536 & $55.342 \mathrm{E} ; 21.045 \mathrm{~S}$ & 1200 & 2010 and 2011 \\
\hline 53 & 97404520 & $55.337 \mathrm{E} ; 21.265 \mathrm{~S}$ & 5 & 2006 and 2007 \\
\hline 54 & 97419380 & $55.687 \mathrm{E} ; 21.217 \mathrm{~S}$ & 2245 & 2010 and 2011 \\
\hline 55 & 97418110 & $55.528 \mathrm{E} ; 20.892 \mathrm{~S}$ & 8 & 2010 and 2011 \\
\hline 56 & 97410202 & $55.693 \mathrm{E} ; 21.007 \mathrm{~S}$ & 16 & 2010 and 2011 \\
\hline 57 & 97420180 & $55.587 \mathrm{E} ; 20.968 \mathrm{~S}$ & 556 & 2010 and 2011 \\
\hline 58 & 97404540 & $55.38 \mathrm{E} ; 21.265 \mathrm{~S}$ & 19 & 2010 and 2011 \\
\hline 59 & $9741555^{\circ}$ & $55.24 \mathrm{E} ; 21.052 \mathrm{~S}$ & 147 & 2010 and 2011 \\
\hline
\end{tabular}

Table A.2: Metadata of the weather stations. The label is the unique identifier within this project. The labels on the geographic map in figure 6.16 refer to these. The ID is the unique identifier of a station within the Météo-France network. The years specify the time window of the temperature record that was used for the analysis. For further description see section 6.5. 


\begin{tabular}{|c|c|c|c|c|c|c|}
\hline Label & $\langle T\rangle\left[{ }^{\circ} \mathrm{C}\right]$ & Deviation $\left[{ }^{\circ} \mathrm{C}\right]$ at o $\mathrm{cm}$ & at $5 \mathrm{~cm}$ & at $10 \mathrm{~cm}$ & at $15 \mathrm{~cm}$ & at $20 \mathrm{~cm}$ \\
\hline oo & 22.53 & 0.52 & 0.82 & 1.02 & 1.02 & 0.82 \\
\hline $\mathrm{O1}$ & 20.57 & 0.77 & 1.07 & 1.17 & 1.07 & 0.97 \\
\hline 02 & 23.80 & 0.55 & 1.05 & 1.05 & 0.95 & 0.75 \\
\hline 03 & 16.44 & 1.39 & 1.89 & 1.99 & 1.89 & 1.79 \\
\hline 04 & 19.22 & 1.01 & 1.31 & 1.42 & 1.31 & 1.31 \\
\hline 05 & 26.17 & 0.68 & 0.89 & 0.99 & 0.79 & 0.89 \\
\hline 06 & 18.76 & 0.87 & 1.28 & 1.38 & 1.38 & 1.28 \\
\hline 07 & 13.92 & 1.19 & 1.39 & 1.39 & 1.39 & 1.29 \\
\hline o8 & 26.65 & 0.51 & 1.01 & 0.91 & 0.81 & 0.71 \\
\hline 09 & $25 \cdot 33$ & 0.62 & 1.02 & 1.12 & 1.02 & 1.02 \\
\hline 10 & $25 \cdot 41$ & 0.24 & 0.74 & 0.94 & 0.74 & 0.74 \\
\hline 11 & 15.41 & 1.01 & 1.41 & 1.51 & 1.31 & 1.21 \\
\hline 12 & 22.43 & 0.81 & 1.31 & 1.41 & 1.31 & 1.31 \\
\hline 13 & 25.61 & 1.15 & 1.65 & 1.55 & 1.45 & 1.45 \\
\hline 14 & 16.48 & 1.25 & 1.45 & 1.55 & 1.45 & 1.45 \\
\hline 15 & 18.84 & 0.79 & 1.19 & 1.39 & 1.29 & 1.29 \\
\hline 16 & 20.38 & 0.56 & 1.16 & 1.36 & 1.36 & 1.26 \\
\hline 17 & $23 \cdot 52$ & 0.42 & 1.03 & 1.13 & 1.03 & 0.93 \\
\hline 18 & $27 \cdot 33$ & 0.73 & 0.93 & 0.93 & 0.83 & 0.73 \\
\hline 19 & 18.61 & 0.92 & 1.32 & 1.32 & 1.22 & 1.12 \\
\hline 20 & 26.64 & 0.42 & 0.92 & 0.92 & 0.82 & 0.92 \\
\hline 21 & 27.27 & 0.69 & 1.09 & 1.19 & 1.09 & 1.09 \\
\hline 22 & 26.94 & 0.92 & 1.12 & 1.12 & 1.02 & 0.92 \\
\hline 23 & 26.05 & 0.30 & 0.80 & 0.90 & 0.90 & 0.80 \\
\hline 24 & 25.01 & 0.24 & 0.84 & 1.04 & 0.84 & 0.74 \\
\hline 25 & 21.84 & 0.50 & 0.90 & 1.20 & 1.10 & 1.00 \\
\hline 26 & 23.01 & 0.74 & 1.14 & 1.04 & 1.14 & 0.94 \\
\hline 27 & 25.89 & 0.47 & 0.87 & 0.97 & 0.87 & 0.77 \\
\hline 28 & 24.16 & 0.79 & 1.29 & 1.39 & 1.39 & 1.29 \\
\hline 29 & 20.08 & 0.96 & 1.16 & 1.26 & 1.06 & 1.16 \\
\hline 30 & $25 \cdot 75$ & 0.41 & 0.81 & 1.01 & 0.91 & 1.01 \\
\hline 31 & 20.44 & 0.90 & 1.40 & 1.40 & 1.40 & 1.30 \\
\hline 32 & 26.60 & 0.56 & 0.96 & 0.86 & 0.76 & 0.76 \\
\hline 33 & $25 \cdot 59$ & 0.46 & 1.06 & 0.96 & 0.96 & 0.96 \\
\hline 34 & 24.49 & 0.56 & 0.96 & 1.06 & 1.06 & 0.96 \\
\hline
\end{tabular}




\begin{tabular}{llrrrrr}
\hline Label & $\langle T\rangle\left[{ }^{\circ} \mathrm{C}\right]$ & Deviation $\left[{ }^{\circ} \mathrm{C}\right]$ at $\mathrm{O} \mathrm{cm}$ & at $5 \mathrm{~cm}$ & at $10 \mathrm{~cm}$ & at $15 \mathrm{~cm}$ & at $20 \mathrm{~cm}$ \\
\hline 35 & 25.60 & 0.56 & 0.96 & 0.96 & 0.96 & 0.86 \\
36 & 22.14 & 0.81 & 1.21 & 1.21 & 1.21 & 1.11 \\
37 & 25.66 & 0.39 & 0.79 & 1.09 & 0.89 & 0.79 \\
38 & 25.55 & 0.40 & 0.70 & 0.90 & 0.80 & 0.80 \\
39 & 25.61 & 0.34 & 0.84 & 0.84 & 0.84 & 0.74 \\
40 & 24.78 & 0.48 & 0.88 & 1.08 & 0.98 & 0.98 \\
41 & 23.68 & 0.57 & 0.97 & 0.97 & 1.07 & 0.97 \\
42 & 23.90 & 0.45 & 0.95 & 1.05 & 0.85 & 0.85 \\
43 & 22.04 & 0.40 & 0.90 & 1.11 & 1.01 & 0.90 \\
44 & 25.93 & 0.12 & 0.82 & 0.82 & 0.72 & 0.62 \\
45 & 21.11 & 1.03 & 1.13 & 1.13 & 1.13 & 1.13 \\
46 & 15.06 & 1.16 & 1.56 & 1.56 & 1.56 & 1.36 \\
47 & 15.98 & 1.14 & 1.44 & 1.54 & 1.54 & 1.34 \\
48 & 21.45 & 0.69 & 0.99 & 1.09 & 0.99 & 0.89 \\
49 & 20.48 & 0.75 & 1.16 & 1.06 & 1.06 & 0.86 \\
50 & 15.59 & 0.83 & 1.03 & 0.93 & 0.73 & 0.63 \\
51 & 17.43 & 0.80 & 1.20 & 1.10 & 0.90 & 0.80 \\
52 & 17.33 & 1.30 & 1.50 & 1.60 & 1.40 & 1.40 \\
53 & 26.23 & 0.53 & 1.33 & 1.33 & 1.33 & 1.23 \\
54 & 13.41 & 0.71 & 1.31 & 1.51 & 1.41 & 1.31 \\
55 & 26.46 & 0.70 & 1.10 & 1.00 & 0.90 & 0.90 \\
56 & 26.09 & 0.36 & 0.86 & 0.86 & 0.86 & 0.96 \\
57 & 21.49 & 0.55 & 0.85 & 0.75 & 0.75 & 0.55 \\
58 & 26.43 & 0.33 & 1.13 & 1.03 & 0.93 & 0.83 \\
59 & 25.45 & 0.30 & 0.80 & 0.90 & 0.90 & 0.90
\end{tabular}

Table A.3: Numerical values of the results of section 6.7. The label is the unique identifier within this project. $\langle T\rangle\left[{ }^{\circ} \mathrm{C}\right]$ is the average air temperature of the temperature record in the time window specified in tabular A.2. For the definition of the deviation see section 6.7. For visualizations of this dataset see figure 6.16 and figure 6.14. 


\section{BIBLIOGRAPHY}

[1] S. Roy and J. Chattopadhyay. "Towards a resolution of 'the paradox of the plankton': A brief overview of the proposed mechanisms." In: Ecological Complexity 4.1-2 (2007), pp. 26-33.

[2] C. Taylor, D. Fudenberg, A. Sasaki, and M.A. Nowak. "Evolutionary Game Dynamics in Finite Populations." In: Bulletin of Mathematical Biology 66.6 (2004), pp. 1621-1644.

[3] M.A. Nowak, A. Sasaki, C. Taylor, and D. Fudenberg. "Emergence of cooperation and evolutionary stability in finite populations." In: Nature 428.6983 (2004), pp. 646-650.

[4] A. Traulsen, J.C. Claussen, and C. Hauert. "Coevolutionary Dynamics: From Finite to Infinite Populations." In: Physical Review Letters 95.23 (2005), p. 238701.

[5] A. Traulsen, M.A. Nowak, and J.M. Pacheco. "Stochastic dynamics of invasion and fixation." In: Physical Review E: Statistical, Nonlinear, and Soft Matter Physics 74.1 (2006), p. 011909.

[6] A. Traulsen, J.C. Claussen, and C. Hauert. "Coevolutionary dynamics in large, but finite populations." In: Physical Review E 74.011901 (2006).

[7] A. Traulsen, J.C. Claussen, and C. Hauert. "Stochastic differential equations for evolutionary dynamics with demographic noise and mutations." In: Physical Review E 85.4 (2012), p. 041901.

[8] M. Assaf, M. Mobilia, and E. Roberts. "Cooperation Dilemma in Finite Populations under Fluctuating Environments." In: Physical Review Letters 111.23 (2013), p. 238101.

[9] P. Ashcroft, P.M. Altrock, and T. Galla. "Fixation in finite populations evolving in fluctuating environments." In: Journal of The Royal Society Interface 11.100 (2014).

[10] W. Huang, C. Hauert, and A. Traulsen. "Stochastic game dynamics under demographic fluctuations." In: Proceedings of the National Academy of Sciences 112.29 (2015), pp. 9064-9069.

[11] G.W. A. Constable, T. Rogers, A.J. McKane, and C.E. Tarnita. "Demographic noise can reverse the direction of deterministic selection." In: Proceedings of the National Academy of Sciences 113.32 (2016), E4745-E4754.

[12] M.A. Nowak and R.M. May. "Evolutionary games and spatial chaos." In: Nature 359.6398 (1992), pp. 826-829. 
[13] C. Hauert and M. Doebeli. "Spatial structure often inhibits the evolution of cooperation in the snowdrift game." In: Nature 428.6983 (2004), pp. 643-646.

[14] F.C. Santos and J.M. Pacheco. "Scale-Free Networks Provide a Unifying Framework for the Emergence of Cooperation." In: Physical Review Letters 95.9 (2005), p. 098104.

[15] G. Szabó and G. Fáth. "Evolutionary games on graphs." In: Physics Reports 446 (2007), pp. 97-216.

[16] H. Ohtsuki, C. Hauert, E. Lieberman, and M.A. Nowak. "A simple rule for the evolution of cooperation on graphs and social networks." In: Nature 441.7092 (2006), pp. 502-505.

[17] M. Perc. "Coherence resonance in a spatial prisoner's dilemma game." In: New Journal of Physics 8.2 (2006), p. 22.

[18] C.P. Roca, J.A. Cuesta, and A. Sánchez. "Evolutionary game theory: Temporal and spatial effects beyond replicator dynamics." In: Physics of Life Reviews 6.4 (2009), pp. 208-249.

[19] T. Konno. "A condition for cooperation in a game on complex networks." In: Journal of Theoretical Biology 269.1 (2011), pp. 224-233.

[20] W. Huang, B. Werner, and A. Traulsen. "The impact of random frequency-dependent mutations on the average population fitness." In: BMC Evolutionary Biology 12.160 (2012).

[21] B. Allen and D.I.S. Rosenbloom. "Mutation Rate Evolution in Replicator Dynamics." In: Bulletin of Mathematical Biology 74.11 (2012), pp. 2650-2675.

[22] D. Foster and P. Young. "Stochastic evolutionary game dynamics." In: Theoretical Population Biology 38.2 (1990), pp. 219-232.

[23] D. Fudenberg and C. Harris. "Evolutionary dynamics with aggregate shocks." In: Journal of Economic Theory 57.2 (1992), pp. $420-441$.

[24] J. Hofbauer and L.A. Imhof. "Time averages, recurrence and transience in the stochastic replicator dynamics." In: The Annals of Applied Probability 19.4 (2009), pp. 1347-1368.

[25] A. Traulsen, T. Röhl, and H.G. Schuster. "Stochastic Gain in Population Dynamics." In: Physical Review Letters 93.2 (2004), p. 028701.

[26] B. Houchmandzadeh and M. Vallade. "Selection for altruism through random drift in variable size populations." In: BMC Evolutionary Biology 12.61 (2012).

[27] B. Houchmandzadeh. "Fluctuation driven fixation of cooperative behavior." In: Biosystems 127 (2015), pp. 6o-66. 
[28] C.S. Gokhale and C. Hauert. "Eco-evolutionary dynamics of social dilemmas." In: Theoretical Population Biology 111 (2016), pp. $28-42$.

[29] E.G. Leigh. "The average lifetime of a population in a varying environment." In: Journal of Theoretical Biology 90.2 (1981), pp. 213-239.

[30] R. Lande. "Risks of Population Extinction from Demographic and Environmental Stochasticity and Random Catastrophes." In: The American Naturalist 142.6 (1993), pp. 911-927.

[31] P. Foley. "Predicting Extinction Times from Environmental Stochasticity and Carrying Capacity." In: Conservation Biology 8.1 (1994), pp. 124-137.

[32] O. Ovaskainen and B. Meerson. "Stochastic models of population extinction." In: Trends in Ecology and Evolution 25.11 (2010), pp. 643-652.

[33] S.J. Schreiber. "Interactive effects of temporal correlations, spatial heterogeneity and dispersal on population persistence." In: Proceedings of the Royal Society of London B: Biological Sciences 277.1689 (2010), pp. 1907-1914.

[34] L.M. Morales. "Viability in a pink environment: why "white noise" models can be dangerous." In: Ecology Letters 2.4 (1999), pp. 228-232.

[35] M. Heino, J. Ripa, and V. Kaitala. "Extinction risk under coloured environmental noise." In: Ecography 23.2 (2000), pp. 177-184.

[36] C.C. Wilmers, E. Post, and A. Hastings. "A Perfect Storm: The Combined Effects on Population Fluctuations of Autocorrelated Environmental Noise, Age Structure, and Density Dependence." In: The American Naturalist 169.5 (2007), pp. 673683 .

[37] M. Schwager, K. Johst, and F. Jeltsch. "Does Red Noise Increase or Decrease Extinction Risk? Single Extreme Events versus Series of Unfavorable Conditions." In: The American Naturalist 167.6 (2006), pp. 879-888.

[38] M. Heino and M. Sabadell. "Influence of coloured noise on the extinction risk in structured population models." In: Biological Conservation 110.3 (2003), pp. 315-325.

[39] L. Ruokolainen, A. Lindén, V. Kaitala, and M.S. Fowler. "Ecological and evolutionary dynamics under coloured environmental variation." In: Trends in Ecology E Evolution 24.10 (2009), pp. 555-563. 
[40] J.V. Greenman and T.G. Benton. "The impact of environmental fluctuations on structured discrete time population models: Resonance, synchrony and threshold behaviour." In: Theoretical Population Biology 68.4 (2005), pp. 217-235.

[41] A. Kamenev, B. Meerson, and B. Shklovskii. "How Colored Environmental Noise Affects Population Extinction." In: Physical Review Letters 101 (2008), p. 268103.

[42] P.M. Altrock and A. Traulsen. "Fixation times in evolutionary games under weak selection." In: New Journal of Physics 11 (2009), p. 013012.

[43] J.W. Baron and T. Galla. "Sojourn times and fixation dynamics in multi-player games with fluctuating environments." In: arXiv 1612.05530 [q-bio.PE] (2016).

[44] C. Van den Broeck, J.M.R. Parrondo, R. Toral, and R. Kawai. "Nonequilibrium phase transitions induced by multiplicative noise." In: Physical Review E 55.4 (1997), pp. 4084-4094.

[45] R. Toral. "Noise-induced transitions vs. noise-induced phase transitions." In: AIP Conf. Proc. 1332 (2011), pp. 145-154.

[46] W. Horsthemke and R. Lefever. Noise-Induced Transitions: Theory and Applications in Physics, Chemistry, and Biology. Ed. by Hermann Haken. Vol. 15. Springer Series in Synergetics. Springer, 1984.

[47] A. Lipshtat, A. Loinger, N.Q. Balaban, and O. Biham. "Genetic Toggle Switch without Cooperative Binding." In: Physical Review Letters 96.18 (18 2006), p. 188101.

[48] T. Biancalani and M. Assaf. "Genetic Toggle Switch in the Absence of Cooperative Binding: Exact Results." In: Physical Review Letters 115.20 (2015), p. 208101.

[49] Charles Darwin. The Descent of Man, and Selection in Relation to Sex. John Murray, 1871.

[50] J. Maynard Smith and G.R. Price. "The Logic of Animal Conflict." In: Nature 246.5427 (1973), pp. 15-18.

[51] H. Kokko, S.C. Griffith, and S.R. Pryke. "The hawk-dove game in a sexually reproducing species explains a colourful polymorphism of an endangered bird." In: Proceedings of the Royal Society B 281.1793 (2014).

[52] J. Gore, H. Youk, and A. van Oudenaarden. "Snowdrift game dynamics and facultative cheating in yeast." In: Nature 459 (2009), pp. 253-256.

[53] P.E. Turner and L. Chao. "Escape from Prisoner's Dilemma in RNA Phage $\Phi 6 . "$ In: The American Naturalist 161.3 (2003), pp. 497-505. 
[54] P.D. Taylor and L.B. Jonker. "Evolutionary stable strategies and game dynamics." In: Mathematical Biosciences 40.1-2 (1978), pp. $145-156$.

[55] P.A.P. Moran. "Random processes in genetics." In: Mathematical Proceedings of the Cambridge Philosophical Society 54.1 (1958), pp. 6o-71.

[56] D. Robinson and D. Goforth. The topology of the $2 \times 2$ games : a new periodic table. Routledge, 2005.

[57] D. Goforth and D. Robinson. "Effective choice in all the symmetric $2 \times 2$ games." In: Synthese 187 (2012), pp. 579-605.

[58] B. Bruns. "Names for Games: Locating 2 x 2 Games." In: Games 6.4 (2015), pp. 495-520.

[59] F. Stollmeier and J. Nagler. "Unfair and Anomalous Evolutionary Dynamics from Fluctuating Payoffs." In: Physical Review Letters 120.5 (2018), p. 058101.

[6o] A. Traulsen and M.A. Nowak. "Evolution of cooperation by multilevel selection." In: Proceedings of the National Academy of Sciences 103.29 (2006), pp. 10952-10955.

[61] L. Lehmann, L. Keller, S. West, and D. Roze. "Group selection and kin selection: Two concepts but one process." In: Proceedings of the National Academy of Sciences 104.16 (2007), pp. 67366739 .

[62] M.A. Nowak. "Five Rules for the Evolution of Cooperation." In: Science 314.5805 (2006), pp. 1560-1563.

[63] L. Gammaitoni, P. Hänggi, P. Jung, and F. Marchesoni. "Stochastic resonance." In: Reviews of Modern Physics 70.1 (1998), pp. 223287 .

[64] J. Tanimoto. "Promotion of cooperation by payoff noise in a 2 x 2 game." In: Physical Review E 76.4 (2007), p. 041130.

[65] R.C. Lewontin and D. Cohen. "On Population Growth in a randomly varying Environment." In: Proceedings of the National Academy of Sciences 62.4 (1969), pp. 1056-1060.

[66] O. Peters. “Optimal Leverage from Non-Ergodicity." In: Quantitative Finance 11.11 (2011), pp. 1593-1602.

[67] O. Peters and W. Klein. "Ergodicity Breaking in Geometric Brownian Motion." In: Physical Review Letters 110.100603 (2013).

[68] P.D. Taylor and L.B. Jonker. "Evolutionary stable strategies and game dynamics." In: Mathematical Biosciences 40.1 (1978), pp. $145^{-156 .}$

[69] J.M. Smith. Evolution and the Theory of Games. Cambridge university press, 1982. 
[70] M. Pigliucci. "Evolution of phenotypic plasticity: where are we going now?" In: Trends in Ecology E Evolution 20.9 (2005), pp. $481-486$.

[71] T.C. Bergstrom. "On the evolution of hoarding, risk-taking, and wealth distribution in nonhuman and human populations." In: Proceedings of the National Academy of Sciences 111 (2014), pp. 10860-10867.

[72] J. Stachurski and V. Martin. "Computing the Distributions of Economic Models via Simulation." In: Econometrica 76.2 (2008), pp. $443-450$.

[73] E. Lieberman, C. Hauert, and M.A. Nowak. "Evolutionary dynamics on graphs." In: Nature 433.7023 (2005), pp. 312-316.

[74] M.H. Vainstein, A.T.C. Silva, and J.J. Arenzon. "Does mobility decrease cooperation?" In: Journal of Theoretical Biology 244.4 (2007), pp. 722-728.

[75] F. Schweitzer and L. Behera. "Optimal migration promotes the outbreak of cooperation in heterogeneous populations." In: Advances in Complex Systems 15.1 (2012), p. 1250059.

[76] F.C. Santos, J.M. Pacheco, and T. Lenaerts. "Evolutionary dynamics of social dilemmas in structured heterogeneous populations." In: Proceedings of the National Academy of Sciences 103.9 (2006), pp. 3490-3494.

[77] F.C. Santos, M.D. Santos, and J.M. Pacheco. "Social diversity promotes the emergence of cooperation in public goods games." In: Nature 454.7201 (2008), pp. 213-216.

[78] F. Stollmeier. "[Re] A simple rule for the evolution of cooperation on graphs and social networks." In: ReScience 3.1 (2017).

[79] B. Allen and M.A. Nowak. "Games on graphs." In: EMS Surveys in Mathematical Sciences 1.1 (2014), pp. 113-151.

[8o] Y.-T. Chen. "Sharp benefit-to-cost rules for the evolution of cooperation on regular graphs." In: Annals of Applied Probability 23.2 (2013), pp. 637-664.

[81] P.D. Taylor, T. Day, and G. Wild. "Evolution of cooperation in a finite homogeneous graph." In: Nature 447.7143 (2007), pp. 469-472.

[82] B. Allen, G. Lippner, Y.-T. Chen, B. Fotouhi, N. Momeni, S.-T. Yau, and M.A. Nowak. "Evolutionary dynamics on any population structure." In: Nature 544.7649 (2017), pp. 227-230.

[83] B. Sinervo and C.M. Lively. "The rock-paper-scissors game and the evolution of alternative male strategies." In: Nature 380 (1996), pp. 240-243. 
[84] F. Schieving and H. Poorter. "Carbon gain in a multispecies canopy: the role of specific leaf area and photosynthetic nitrogenuse efficiency in the tragedy of the commons." In: New Phytologist 143.1 (1999), pp. 201-211.

[85] M. Gersani, J.S. Brown, E.E. O'Brien, G.M. Maina, and Z. Abramsky. "Tragedy of the commons as a result of root competition." In: Journal of Ecology 89.4 (2001), pp. 660-669.

[86] B.C. Kirkup and M.A. Riley. "Antibiotic-mediated antagonism leads to a bacterial game of rock-paper-scissors in vivo." In: Nature 428.6981 (2004), pp. 412-414.

[87] A.S. Griffin, S.A. West, and A. Buckling. "Cooperation and competition in pathogenic bacteria." In: Nature 430.7003 (2004), pp. 1024-1027.

[88] L.A. Dugatkin, M. Perlin, J. Scott Lucas, and R. Atlas. “Groupbeneficial traits, frequency-dependent selection and genotypic diversity: an antibiotic resistance paradigm." In: Proceedings of the Royal Society B 272.1558 (2005), pp. 79-83.

[89] E.A. Yurtsev, H.X. Chao, M.S. Datta, T. Artemova, and J. Gore. "Bacterial cheating drives the population dynamics of cooperative antibiotic resistance plasmids." In: Molecular Systems Biology 9.683 (2013).

[9o] M.H. Perlin et al. "Protection of Salmonella by ampicillin-resistant Escherichia coli in the presence of otherwise lethal drug concentrations." In: Proceedings of the Royal Society of London B: Biological Sciences 276.1674 (2009), pp. 3759-3768.

[91] R.A. Sorg, L. Lin, G.S. van Doorn, M. Sorg, J. Olson, V. Nizet, and J.-W. Veening. "Collective Resistance in Microbial Communities by Intracellular Antibiotic Deactivation." In: PLoS Biology 14.12 (2016), e2000631.

[92] M.G.J. de Vos, M. Zagorski, A. McNally, and T. Bollenbach. "Interaction networks, ecological stability, and collective antibiotic tolerance in polymicrobial infections." In: Proceedings of the National Academy of Sciences 114.40 (2017), pp. 10666-10671.

[93] P. Chesson. "Mechanisms of maintenance of species diversity." In: Annual Review of Ecology and Systematics 31 (2000), pp. 343366.

[94] J. Tan, C.K. Kelly, and L. Jiang. "Temporal niche promotes biodiversity during adaptive radiation." In: Nature Communications 4.2102 (2013).

[95] R. Durrett and S. Levin. "Spatial Aspects of Interspecific Competition." In: Theoretical Population Biology 53.1 (1998), pp. 3043 . 
[96] B. Kerr, M.A. Riley, M.W. Feldman, and B.J.M. Bohannan. "Local dispersal promotes biodiversity in a real-life game of rockpaper-scissors." In: Nature 418.6894 (2002), pp. 171-174.

[97] E.D. Kelsic, J. Zhao, K. Vetsigian, and R. Kishony. “Counteraction of antibiotic production and degradation stabilizes microbial communities." In: Nature 521.7553 (2015), pp. 516-519.

[98] R.E. Lenski and S.E. Hattingh. "Coexistence of two competitors on one resource and one inhibitor: A chemostat model based on bacteria and antibiotics." In: Journal of Theoretical Biology 122.1 (1986), pp. 83-93.

[99] S.-B. Hsu and P. Waltman. "A survey of mathematical models of competition with an inhibitor." In: Mathematical Biosciences 187.1 (2004), pp. 53-91.

[10o] D.I. Andersson and D. Hughes. "Antibiotic resistance and its cost: is it possible to reverse resistance?" In: Nature Reviews Microbiology 8.4 (2010), pp. 260-271.

[101] E. Geisinger and R.R. Isberg. "Antibiotic Modulation of Capsular Exopolysaccharide and Virulence in Acinetobacter baumannii." In: PLoS Pathogens 11.2 (2015), e1004691.

[102] K. Gupta, S. Singh, and M.L. van Hoek. "Short, Synthetic Cationic Peptides Have Antibacterial Activity against Mycobacterium smegmatis by Forming Pores in Membrane and Synergizing with Antibiotics." In: Antibiotics 4.3 (2015), pp. 358-378.

[103] I. Brook. "Inoculum Effect." In: Reviews of Infectious Diseases 11.3 (1989), pp. 361-368.

[104] K.I. Udekwu, N. Parrish, P. Ankomah, F. Baquero, and B.R. Levin. "Functional relationship between bacterial cell density and the efficacy of antibiotics." In: Journal of Antimicrobial Chemotherapy 63.4 (2009), pp. 745-757.

[105] V.H. Tam, A. Louie, M.R. Deziel, W. Liu, and G.L. Drusano. "The Relationship between Quinolone Exposures and Resistance Amplification Is Characterized by an Inverted U: a New Paradigm for Optimizing Pharmacodynamics To Counterselect Resistance." In: Antimicrobial Agents and Chemotherapy 51.2 (2007), pp. 744-747.

[106] A.A. Firsov, M.V. Smirnova, I.Yu. Lubenko, S.N. Vostrov, Y.A. Portnoy, and S.H. Zinner. "Testing the mutant selection window hypothesis with Staphylococcus aureus exposed to daptomycin and vancomycin in an in vitro dynamic model." In: Journal of Antimicrobial Chemotherapy 58.6 (2006), pp. 1185-1192.

[107] T. Day and A.F. Read. "Does High-Dose Antimicrobial Chemotherapy Prevent the Evolution of Resistance?" In: PLoS Computational Biology 12.1 (2016), e1004689. 
[108] G.D. Wright. "Bacterial resistance to antibiotics: Enzymatic degradation and modification." In: Advanced Drug Delivery Reviews 57.10 (2005), pp. 1451-1470.

[109] M.E. Hibbing, C. Fuqua, M.R. Parsek, and S.B. Peterson. "Bacterial competition: surviving and thriving in the microbial jungle." In: Nature Reviews 8 (2009), pp. 15-25.

[110] K. Vetsigian, R. Jajoo, and R. Kishony. "Structure and Evolution of Streptomyces Interaction Networks in Soil and In Silico." In: PLOS Biology 9.10 (2011), pp. 1-12.

[111] O.X. Cordero, H. Wildschutte, B. Kirkup, S. Proehl, L. Ngo, F. Hussain, F. Le Roux, T. Mincer, and M.F. Polz. "Ecological Populations of Bacteria Act as Socially Cohesive Units of Antibiotic Production and Resistance." In: Science 337.6099 (2012), pp. 1228-1231.

[112] H.C. Davison, J.C. Low, and M.E.J. Woolhouse. "What is antibiotic resistance and how can we measure it?" In: Trends in Microbiology 8.12 (2000), pp. 554-559.

[113] A.E. Bashirov, E.M. Kurpınar, and A. Özyapıcı. "Multiplicative calculus and its applications." In: Journal of Mathematical Analysis and Applications 337.1 (2008), pp. 36-48.

[114] A.E. Bashirov, E. Misırlı, Y. Tandoğdu, and A. Özyapıc1. “On modeling with multiplicative differential equations." In: Applied Mathematics-A Journal of Chinese Universities 26.4 (2011), pp. 425-438.

[115] N. Arley. On the theory of stochastic processes and their application to the theory of cosmic radiation. Wiley, 1948.

[116] A. Azzalini. "A Class of Distributions Which Includes the Normal Ones." In: Scandinavian Journal of Statistics 12.2 (1985), pp. 171178.

[117] J.W. Golden and D.L. Riddle. "The Caenorhabditis elegans Dauer Larva: Developmental Effects of Pheromone, Food, and Temperature." In: Developmental Biology 102.2 (1984), pp. 368378.

[118] L. Byerly, R.C. Cassada, and R.L. Russell. "The Life Cycle of the Nematode Caenorhabditis elegans." In: Developmental Biology 51 (1976), pp. 23-33.

[119] D. Muschiol, F. Schroeder, and W. Traunspurger. "Life cycle and population growth rate of Caenorhabditis elegans studied by a new method." In: BMC Ecology 9.1 (2009), p. 14.

[120] M.L. Begasse, M. Leaver, F. Vazquez, S.W. Grill, and A.A. Hyman. "Temperature Dependence of Cell Division Timing Accounts for a Shift in the Thermal Limits of C. elegans and C. briggsae." In: Cell Reports 10.5 (2015), pp. 647-653. 
[121] R.C. Venette and H. Ferris. "Thermal constraints to population growth of bacterial-feeding nematodes." In: Soil Biology and Biochemistry 29.1 (1997), pp. 63-74.

[122] D. Ramot, B.L. MacInnis, H.-C. Lee, and M.B. Goodman. "Thermotaxis is a Robust Mechanism for Thermoregulation in Caenorhabditis elegans Nematodes." In: The Journal of Neuroscience 28.47 (2008), pp. 12546-12557.

[123] M.A. Félix and F. Duveau. "Population dynamics and habitat sharing of natural populations of Caenorhabditis elegans and C. briggsae." In: BMC Biology 10.59 (2012).

[124] G.S. Campbell and J.M. Norman. An Introduction to Environmental Biophysics. Springer, 1998.

[125] N.H. Abu-Hamdeh. "Thermal Properties of Soils as affected by Density and Water Content." In: Biosystems Engineering 86.1 (2003), pp. 97-102.

[126] D. Goutx, F. Bonnardot, G. Eugénie, and G. Jumaux, eds. Bulletin climatologique 2016 (2016).

[127] M. Baym, T.D. Lieberman, E.D. Kelsic, R. Chait, R. Gross, I. Yelin, and R. Kishony. "Spatiotemporal microbial evolution on antibiotic landscapes." In: Science 353.6304 (2016), pp. 11471151.

[128] S. Luo and A.S. Perelson. "The challenges of modelling antibody repertoire dynamics in HIV infection." In: Philosophical Transactions of the Royal Society B 370.1676 (2015).

[129] N.J. Dimmock and A.J. Easton. "Defective Interfering Influenza Virus RNAs: Time To Reevaluate Their Clinical Potential as Broad-Spectrum Antivirals?" In: Journal of Virology 88.10 (2014), pp. 5217-5227.

[130] K.S. Korolev, J.B. Xavier, and J. Gore. "Turning ecology and evolution against cancer." In: Nature Reviews Cancer 14 (2014), pp. 371-38o.

[131] S. Quercia et al. "From lifetime to evolution: timescales of human gut microbiota adaptation." In: Frontiers in Microbiology 5 (2014), p. 587. 


\section{ACKNOWLEDGMENTS}

Along the way from the beginning of my work at the institute to the completion of this thesis, I enjoyed the help, support, inspiration and positive influence of several people.

I am grateful to Marc Timme, Theo Geisel, Jan Nagler and Ulrich Parlitz, for scientific advice and critical comments on my unfinished results during the thesis committee meetings.

I like to thank Mark Timme and Theo Geisel for allowing me to be part of the NLD department and the Network Dynamics group, for their support, and for combining scientific events with pleasant activities like skiing, canoeing or hiking.

A very special thanks goes to Jan Nagler, who guided me through this endeavor. Thanks for initiating these fascinating scientific projects, for giving me advise and critical feedback, and for being always available, even while being far away.

The project on the evolution of nematodes would not exist without Mark Leaver. I am grateful for sharing knowledge, ideas and data with me and for inviting me to Dresden to get personally acquainted with the nematodes.

In addition, related to this project I also need to thank the French national meteorological service Météo-France and the weather-service Weather Underground for granting me access to their valuable data.

For proof-reading I like to thank Jan, Fede, and Phine, and for critical comments on layout and design I thank Manuel.

I acknowledge the funding through a PhD fellowship and travel grants provided by the International Max Planck Research School of Physics of Biological and Complex Systems (IMPRS-PBCS), and I acknowledge the organisation of interesting courses and excursions by the Göttingen Graduate School for Neurosciences, Biophysics, and Molecular Biosciences (GGNB).

I thank all present and former members of the NLD department and the Networks Dynamics group for the collaborative atmosphere, for many interesting discussions, exchange of ideas and opinions, kicker tournaments and other relaxing breaks from work, and diverting evenings outside the institute.

A special thanks goes to Manuel for the unique office culture and for our joint projects like our botanical experiments, the cluster usage statistics, the Müsli box progress bar (I think we can call it complete), and the projects that we did not yet complete, the Tierchen and the Abus 85 .

Thanks to all members of the python teaching community, in particular Florencia, Norma and Debshanka, for a series of very nice courses. 
I also like to thank a group of people who I mostly do not know personally, but who indirectly contributed much to this work. This includes everybody who voluntarily works on open knowledge and open software projects without expecting anything except that it may be useful for someone. Among many others, Linux, Python, Numpy, Scipy, and many other Python modules, Inkscape, Sozi, Zim, Wikipedia and Wikimedia Commons were extremely useful for the daily scientific work.

Beyond the people who contributed to the work on the thesis, I also like to thank some people for distracting me from working on the thesis. I thank Kathi, Maria, Kolja, Jan, Florian, Paulina and Kristinë for the Giant Threat, Fede for many nice evenings with movies and music and for some lessons in Taoism, Norma for trips to the lake on sunny days when everybody else preferred to work, Phine for our holidays in a spooky Herrenhaus during the final phase of this thesis, and Enie and Cynthia for entertaining evenings with games and crêpes. 